\title{
Review of genetic diversification of bats in the Caribbean and biogeographic relationships to Neotropical species based on DNA barcodes
}

\begin{tabular}{|r|l|}
\hline Journal: & Genome \\
\hline Manuscript ID & gen-2015-0204.R2 \\
\hline Manuscript Type: & Article \\
\hline Date Submitted by the Author: & 10-Jun-2016 \\
\hline Complete List of Authors: & Lim, Burton; Royal Ontario Museum, Natural History \\
\hline Keyword: & Antilles, Chiroptera, Dominican Republic, Jamaica, Martinique \\
\hline \multicolumn{2}{|l}{} \\
\hline
\end{tabular}

\section{SCHOLARONE ${ }^{\text {m }}$ \\ Manuscripts}




\section{Review of genetic diversification of bats in the Caribbean and biogeographic relationships to Neotropical species based on DNA barcodes}

Burton K. Lim

B.K. Lim, Department of Natural History, Royal Ontario Museum, 100 Queen's Park, Toronto, ON M5S 2C6, Canada.

Corresponding author: Burton K. Lim (burtonl@ rom.on.ca). 


\begin{abstract}
DNA barcoding is helping in discovering high levels of cryptic species and an underestimation of biodiversity in many groups of organisms. Although mammals are arguably the most studied and one of the least speciose taxonomic classes, the rate of species discovery is increasing and biased for small mammals on islands. An earlier study found bats in the Caribbean as a taxonomic and geographic deficiency in the International Barcode of Life initiative to establish a genetic reference database to enable specimen identification to species. Recent surveys in Dominican Republic, Jamaica, and Martinique have documented and barcoded half of the 58 bat species known from the Caribbean. I analyze all available barcode data of Caribbean bats to investigate biogeography and cryptic species in the Neotropical region. Analysis of the mitochondrial DNA gene cytochrome c oxidase subunit 1 results in a phylogenetic tree with all but one species as well-supported and reciprocally monophyletic. With a broader sampling across the Neotropics, there are also divergent lineages that exhibit biogeographic structuring: (1) phylogenetic split between northern and southern Dominican Republic in 3 species, (2) 2 taxa with cryptic species associated with higher degree of island endemism, (3) populations of 2 widely distributed species with deep divergence between the Caribbean and North and Central America, and (4) 1 species in the Caribbean with affinities to taxa in South America.
\end{abstract}

Keywords: Antilles, Chiroptera, Dominican Republic, Jamaica, Martinique. 


\section{Introduction}

Surprisingly for a well-known and studied group of animals, about 300 new species of mammals have been described in the last decade (Reeder et al. 2007). Furthermore, the rate of discovery is biased to smaller mammals on islands, although most of this has been documented in the Old World tropics. One of the primary factors is the increased use of molecular methods, in particular DNA sequencing, that is uncovering cryptic species in many widely distributed but morphologically similar taxa (Baker and Bradley 2006). DNA barcoding of the cytochrome c oxidase subunit 1 (COI) gene for specimen identification and species discovery (Hebert et al. 2003 ) is at the forefront. For example, a recent study on bats in Southeast Asia estimated a 50\% increase in undiscovered species diversity (Francis et al. 2010). By contrast, the Caribbean was noted as a region of the world that was lacking in DNA barcodes of mammals (Lim 2012), contributing to a bias towards new species descriptions from the Paleotropics (Reeder et al. 2007). The majority (79\%) of the 73 species of extant terrestrial mammals in the Caribbean are bats, of which 69\% are endemic (Dávalos and Turvey 2012). Moreover, $42 \%$ of the bat species endemic to the Caribbean are threatened (www.iucnredlist.org). There is an urgent need to document the species diversity in this conservation hotspot, ranked number three in terms of regions in the world with high endemism coupled with high habitat loss and small area (Myers et al. 2000).

The purpose of this study is to summarize DNA barcodes to ascertain the genetic diversity of bats from the Caribbean and compare barcode clusters to currently recognized species. In addition, a more detailed assessment with a broader taxonomic survey of closely related taxa and a wider geographic sampling of continental populations will examine phylogenetic structuring within species and implications for the biogeography of bats in the 
Neotropics. This study complements earlier work on the phylogeny and biogeography of Caribbean bats by Dávalos (2005, 2006, 2007), but with increased geographic sampling on several islands in the Greater Antilles based on recent fieldwork in Jamaica (Lim and Arcila Hernandez 2016) and Dominican Republic (Lim et al. in press) and in the Lesser Antilles based on recently deposited sequences in Genbank (http://www.ncbi.nlm.nih.gov/genbank) from Martinique.

\section{Materials and methods}

DNA barcodes of 29 of the 37 recognized species from Jamaica, Dominican Republic, and Martinique were analyzed in this study, which represents half of the 58 known extant species of bats in the Caribbean (Dávalos and Turvey 2012). This diversity was represented by 246 samples (Table 1), including 81 samples of 10 species from Jamaica as reported by Lim and Arcila Hernandez (2016), 132 samples of 15 species from Dominican Republic as reported by Lim et al. (in press), and 33 samples of 10 species from Martinique deposited on GenBank. The 8 species not included in the study were Ariteus flavescens, Eumops auripendulus, Eumops glaucinus, Lasiurus degelidus, Natalus jamaicensis, Natalus stramineus, Nyctinomops macrotis, and Phyllonycteris aphylla.

Molecular methods followed the DNA barcoding procedure for bats as outlined by Clare et al. (2007). In summary, DNA was isolated using a Phenol/Chloroform extraction procedure with approximately $1 \mathrm{~mm}^{3}$ of tissue (muscle or liver) and an elution volume of $60 \mathrm{ul}$. Bidirectional primers used for recovering the COI mtDNA gene were VF1 (5'TTCTCAACCAACCACAAAGACATTGG-3') and VR1 (5'- 
TAGACTTCTGGGTGGCCAAAGAATCA-3'). The polymerase chain reaction (PCR) consisted of $8.92 \mathrm{ul} \mathrm{H}_{2} \mathrm{O}, 1.25 \mathrm{ul} \mathrm{EH}$ Buffer, $0.5 \mathrm{ul}$ or $10 \mathrm{uM}$ of each primer, $0.28 \mathrm{ul}$ or $10 \mathrm{mM}$ dNTP's (Invitrogen), 0.05 ul taq (Invitrogen), and 1 ul DNA. Amplification conditions were 94C for $2 \mathrm{~min}$; then 36 cycles of $94 \mathrm{C}$ for $30 \mathrm{sec}, 50 \mathrm{C}$ for $30 \mathrm{sec}$, and $72 \mathrm{C}$ for $1 \mathrm{~min} 30 \mathrm{sec}$; followed by $72 \mathrm{C}$ for $3 \mathrm{~min}$; and then a $4 \mathrm{C}$ hold. The PCR product was separated on a $1 \%$ agarose gel at $100 \mathrm{~V}$ for $20 \mathrm{~min}$. The PCR band was excised and placed in a spin column made by cutting the ends of 200-ul filter tips that were placed in 1.5-ml microcentrifuge tubes. These were spun at full speed for $10 \mathrm{~min}$, and $4 \mathrm{ul}$ of this product was used in a sequencing mix of $3 \mathrm{ul} \mathrm{H} 2 \mathrm{O}, 2 \mathrm{ul} 5 \mathrm{x}$ sequencing buffer, $0.5 \mathrm{ul}$ primer, and 0.5ul BigDye. The sequencing reactions and precipitation followed the protocol of Applied Biosystems and were then resuspended in 10ul HiDi formamide and placed on the sequencer. Resultant chromatograms were edited using BioEdit and a single contig created for each specimen.

Sequence alignment was done with Sequencher version 4.8 (Gene Code Corp. 2007), and no internal stop codons were present to indicate pseudo-genes. Genetic distances were calculated using the Kimura 2-parameter model of nucleotide substitutions with pairwise deletion in MEGA6 (Tamura et al. 2013), which is appropriate for closely related taxa (Nei and Kumar 2000) and enables comparison to the similarly mutating mitochondrial cytochrome b gene used by Baker and Bradley (2006) in the genetic species concept for mammals. Phylogenetic trees were constructed using the neighbour-joining distance method, as a quick algorithm for calculating large phylogenies (Kumar and Gadagkar 2000), and maximum likelihood with pairwise deletion and nearest neighbor interchange based on the best-fit nucleotide substitution model with the lowest Baysian Information Criterion as implemented in MEGA6. Topological support in all trees was tested with 500 bootstrap replicates. Trees were 
constructed first for the 246 samples from the Caribbean and then separately for species groups exhibiting phylogenetic structuring, with comparative sequences of closely related taxa downloaded from GenBank (Table S1).

\section{Results}

DNA barcode variation of 29 Caribbean bat species, as summarized by maximum likelihood (based on a general time reversible substitution model with gamma distribution and some invariable sites) and neighbor-joining trees, recovered well-supported $(\geq 95 \%)$ monophyletic clades for all but 1 species (Fig. 1). The exception was Brachyphylla pumila, which was paraphyletic in relation to $B$. cavernarum. Of the 5 intragenera groupings, all have high bootstrap values $(\geq 90 \%)$ except Pteronotus $(\geq 66 \%)$. Several intergeneric relationships are well-supported ( $\geq 95 \%)$ including (Phyllonycteris, Erophylla) and ((Ardops, Phyllops, (Artibeus)). All other higher-level relationships were poorly supported, and there were no natural groupings at the taxonomic level of family. This is not unexpected because the rate of nucleotide substitution of COI typically has phylogenetic signal at the species level, and its appropriateness decreases at higher levels (Hajibabaei et al., 2007).

Kimura 2-parameter interspecific sequence divergence averaged from $33.6 \%$ between Erophylla sezekorni and Lasiurus minor to $1.2 \%$ between Brachyphylla pumila and $B$. cavernarum (Table 2). Average intraspecific divergence ranged from $4.7 \%$ for Macrotus waterhousii to no variation within 8 species. Two species (Natalus major and Erophylla sezekorni) were represented by only 1 sample. 
For broader studies of taxonomic groups across the Neotropics, maximum likelihood tree based on Tamura 3-parameter substitution model with some invariable sites recovered 2 species with deep phylogenetic splits between populations from the Caribbean and Central and North America (Fig. 2). Samples of Eptesicus fuscus from the Dominican Republic have an average sequence divergence of $8 \%$ from those collected from Canada and the United States. Similarly, populations of Tadarida brasiliensis from Martinique are 6\% divergent from Guatemala, Mexico, and United States.

Based on a maximum likelihood analysis with a Tamura 3-parameter substitution model with gamma distribution and some invariable sites, one species has a closer affinity to South America. Although poorly supported, Myotis martiniquensis from Martinique is embedded within a clade of primarily South American occurring taxa (Fig. 2). It has an average sequence divergence of $6.7 \%$ from its poorly supported sister clade of $M$. riparius.

A maximum likelihood tree based on Hasegawa-Kinoshita-Yano substitution model with gamma distribution for three Caribbean endemic genera with COI sequences from more than one island exhibit contrasting levels of genetic distance and taxonomic rank (Fig. 3). Brachyphylla pumila from Dominican Republic is not reciprocally monophyletic with respect to $B$. cavernarum from Martinique, and there is an average sequence divergence of $1.2 \%$. Erophylla bombifrons from Dominican Republic is the sister species of E. sezekorni from Jamaica, and the average sequence divergence is $4.3 \%$. By contrast, the average sequence divergence between populations of Monophyllus redmani from Dominican Republic and Jamaica is $8.1 \%$. The average sequence divergence between the sister species $M$. redmani and $M$. plethodon from Martinique is $14 \%$. 
Based on a maximum likelihood analysis with a best fit Hasegawa-Kinoshhita-Yano substitution model with some invariable sites, there is one widely distributed bat genus that also has phylogeographic patterns associated with individual Greater Antillean islands. Populations of Molossus molossus from Jamaica and Dominican Republic are divergent from each other and other continental populations by about $4 \%$ (Fig 3) as reported recently by Lim et al. (in press).

Based upon maximum likelihood analyses using the Hasegawa-Kinoshhita-Yano substitution model with gamma distribution for Macrotus and Tamura 3-parameter model with some invariable sites for Pteronotus, there is a phylogenetic break between northern and southern Dominican Republic. Pairwise sequence divergence averaged $7.6 \%$ between populations of Macrotus waterhousii, 3.4\% in Pteronotus pusillus, and $1.3 \%$ in P. quadridens (Fig.4 6) as documented in an earlier study (Lim et al. in press).

\section{Discussion}

Phylogenetic relationships of Caribbean bats based on the mitochondrial gene COI identified 4 general biogeographic patterns when compared to a wider sampling across the Neotropics. However, typical systematic reviews over the years have focused primarily at the taxonomic rank of family or below. In combination with different researchers and classifications, this has resulted in an uneven treatment at the species level. A broader study within bats of the Caribbean gives an opportunity to standardize and update the taxonomy with the benefit of applying molecular methods such as DNA barcoding to the suggestion that cryptic species are contributing to the increasing trend of discovering new species of mammals (Baker and Bradley 2006; Reeder et al., 2007). 
Previous studies on the biogeography of bats in the Caribbean have taken a distributional occurrence (Baker and Genoways 1978) or ecological (Presley and Willig 2008) approach. Although there have been some recent studies on individual families (Dávalos 2005, 2006, 2007), a broad phylogenetic approach has not been done for the order Chiroptera, but Dávalos and Turvey (2012) outlined gaps in our knowledge that should be addressed. I present a preliminary overview of genetic diversity in the Caribbean and compare it to current taxonomy and the implications to biogeography of bats in the Neotropics.

Broad phylogeographic structuring is seen in 2 widely distributed species that occur in the Caribbean and primarily on the continental mainland of North America and into Central America. Although both Eptesicus fuscus and Tadarida brasiliensis are distributed in South America, they are not found east of Venezuela into the Amazon basin (Gardner 2008). Geographic sampling of DNA barcoding is abysmal for these 2 species, but average sequence divergence at $\mathrm{COI}$ is $6 \%$ between Caribbean and mainland populations, which is comparable to cytochrome b values for sister species of bats and suggestive of the cessation of gene flow (Baker and Bradley 2006). There are subspecies names applicable to these Caribbean populations: Eptesicus fuscus hispaniolae and Tadarida brasiliensis antillularum.

At shallower levels of divergence, there is also phylogeographic structuring linking Caribbean and Central American populations of Glossophaga soricina and Artibeus jamaicensis. There is a haplotype shared among individuals of A. jamaicensis from Dominican Republic, Jamaica, and the Yucatan Peninsula of Mexico, suggesting ongoing gene flow (Lim and Arcila Hernandez 2016). This result supports an earlier study of restriction site data with shared mtDNA genotypes between the Caribbean and Yucatan (Phillips et al. 1991). For G. soricina, the Jamaican population comprises a well-supported monophyletic clade that is nested within a 
larger geographic clade that includes Central America and South America west of the Andes to the exclusion of populations from east of the Andes (Hoffman and Baker 2001; Lim and Arcila 2016).

However, there is 1 species occurring in the Caribbean that has affinities to South America. Although the deeper phylogenetic relationships are not well supported in the COI tree, Myotis martiniquensis is allied with species occurring primarily in South America, but $M$. riparius does extend into southern Central America (Gardner 2008). These 2 species have an average sequence divergence of $6.7 \%$. Similar results were found using the cytochrome b gene (Larsen et al 2012), although the taxonomic data sets were not identical.

Three endemic Caribbean genera have varying degrees of sequence divergence associated with different islands. The allopatric sister species Erophylla sezekorni from Jamaica and E. bombifrons from Dominican Republic have an average sequence divergence of $4.3 \%$. By contrast, populations of Monophyllus redmani from Jamaica and Dominican Republic are divergent by $8.1 \%$. They are currently recognized as subspecies $M$. r. redmani and $M . r$. clinedaphus, respectively. The sister species is M. plethodon, which is distributed in the Lesser Antilles including Martinique, and divergent by 14\%. In comparison, Brachyphylla cavernarum from Martinique has an average sequence divergence of $1.2 \%$ from $B$. pumila in the Dominican Republic and exhibits a weakly supported paraphyletic arrangement. However, B. cavernarum is larger with a forearm length averaging $>60 \mathrm{~mm}$, and B. pumila is smaller but within the size range of B. nana from Cuba, which it was previously synonymized under (Swanepoel and Genoways 1978). More recently, B. pumila was recognized as a distinct species based on 23 fixed differences in cytochrome b from B. nana (Dávalos 2004). A systematic review is needed to resolve this taxonomic instability. 
Two genera more broadly found throughout the Neotropics also exhibit deep divergence in DNA barcoding sequences associated with Caribbean islands (Lim et al. in press). Earlier molecular studies (Dávalos 2006), combined with recent analysis of echolocation call frequencies (de Thoisy et al. 2014), have identified several cryptic species within the widely distributed Pteronotus parnellii. The nominate species is now restricted to Jamaica, P. pusillus to Hispaniola, and P. portoricensis to Puerto Rico. Similarly, the broadly occurring Molossus molossus also needs to be separated into several species because of a polyphyletic relationship with respect to $M$. rufus and $M$. coibensisas identified in earlier studies of COI variation in the Caribbean (Lim and Arcila Hernandez, 2016; Lim et al. in press). Available names include $M$. milleri for populations in Jamaica and $M$. verrilli in Hispaniola. Individuals from the Lesser Antilles, including the nominal type locality in Martinique, nest within the mainland population of M. molossus.

There is a phylogenetic split between northern and southern Dominican Republic for 3 species of bats as recently reported by Lim et al. (in press) based on COI diversity. Varying degrees of sequence divergence for Macrotus waterhousii (7.6\%), Pteronotus pusillus (3.4\%), and $P$. quadridens (1.3\%) suggest a persistent barrier to gene flow. This geogeographic separation coincides with the Miocene paleo-islands that eventually formed Hispaniola approximately 10 million years ago by tectonic activity and subsequent fluctuations in sea levels (Iturralde-Vinent and MacPhee 1999; Graham 2003). Recent molecular studies of lizards (Gifford, et al. 2004), birds (Townsend et al. 2007), and rodents (Brace et al. 2012) have also recovered this geographic break.

In summary, molecular phylogenetic analysis of Caribbean bats and closely related continental species identify 4 general patterns of biogeography in the Neotropics and the 
discovery of cryptic species diversity. (1) Phylogeographic structuring is seen within some widely distributed species with populations in the Caribbean and Central and North America. (2) Recent systematic revisions identify Lesser Antillean endemics as closely related to taxa in South America. (3) Both Caribbean endemic taxa and more broadly occurring genera have species allopatrically distributed on islands. (4) There is a phylogenetic separation of northern and southern Hispaniolan populations in 3 species of bats.

DNA barcoding and other molecular methods are discovering an underestimation of biodiversity that will contribute to a better understanding of the evolution of bats and other organisms in the Caribbean and throughout the world. However, comprehensive systematic revisions are needed to document this increase in taxonomic diversity This study also highlights a sampling bias towards new species descriptions on islands, primarily in the Paleotropics (Reeder et al. 2007), but there is still much to be discovered and studied in the insular Neotropics. As the third hottest hotspot (Myers et al. 2000), good estimations of biodiversity are necessary for prioritizing conservation initiatives in the Caribbean.

\section{Acknowledgements}

I thank my field and research collaborators in the Caribbean, including Lina Arcila, Livia Loureiro, Nate Upham, Jorge Brocca, and Francois Catzeflis, for contributing specimens to this review paper. Thanks also to the National Environment and Planning Agency in Jamaica and Ministerio de Medio Ambiente y Recursos Naturales in Dominican Republic for research and export permits (No. VAPB-01095). Appreciation is extended to 2 anonymous reviewers and the Associate Editor, Beth Clare, for constructive comments that improved the paper. Liliana 
Dávalos kindly provided collecting locality information for the Dominican Republic. This project was supported by the Life In Crisis: Schad Gallery of Biodiversity Research Fund and the Collections \& Research Fieldwork Fund at the Royal Ontario Museum.

\section{References}

Baker, R.J, and Bradley, R.D. 2006. Speciation in mammals and the genetic species concept. J. Mammal. 87: 643-62.

Baker, R.J., and Genoways, H.H. 1978. Zoogeography of Antillean bats. In Zoogeography of the Caribbean. Edited by F.B. Gill. Spec. Publ., Acad. Nat. Sci., Philadelphia, pp. 53-98.

Brace, S., Barnes, I., Powell, A., Pearson, R., Woolaver, L.G., Thomas, M.G., and Turvey, S.T. 2012. Population history of the Hispaniolan hutia Plagiodontia aedium (Rodentia: Capromyidae): testing the model of ancient differentiation on a geotectonically complex Caribbean island. Mol. Ecol. 21: 2239-2253.

Clare, E.L., Lim, B.K., Engstrom, M.D., Eger, J.L., and Hebert, P.D.N. 2007. DNA barcoding of Neotropical bats: species identification and discovery within Guyana. Mol. Ecol. Notes 7: 184-90.

Dávalos, L.M. 2004. Historical biogeography of the Antilles: earth history and phylogenetics of endemic chiropteran taxa. Ph.D. dissertation. Columbia University, New York, NY.

Dávalos, L.M. 2005. Molecular phylogeny of Funnel-eared bats (Chiroptera: Natalidae), with notes on biogeography and conservation. Mol. Phylogenet. Evol. 37: 91-103. 
Dávalos, L.M. 2006. The geography of diversification in the mormoopids (Chiroptera: Mormoopidae). Biol. J. Linn. Soc. 88: 101-118.

Dávalos, L.M. 2007. Short-faced bats (Phyllostomidae: Stenodermatina): a Caribbean radiation of strict frugivores. J. Biogeogr. 34: 364-375.

Dávalos, L.M., and Turvey, S.T. 2012. West Indian mammals: the old, the new, and the recently extinct. In Bones, clones, and biomes: the history and geography of Recent Neotropical mammals. Edited by B.D. Patterson and L.P. Costa. University of Chicago Press, Chicago, Ill. pp. 157-202.

De Thoisy, B., Pavan, A.C., Delaval, M., Lavergne, A., Luglia, T., Pineau, K., Ruedi, M., Rufray, V., and Catzeflis, F. 2014. Cryptic diversity in common mustached bat Pteronotus cf. parnellii (Mormoopidae) in French Guiana and Brazilian Amapa. Acta Chiropt. 16: 1-13.

Francis, C.M., Borisenko, A.V., Ivanova, N.V., Eger, J.L., Lim, B.K., Guillén-Servent, A., Kruskop, S.V., Mackie, I., and Hebert, P.D.N. 2010. The role of DNA barcodes in understanding and conservation of mammal diversity in Southeast Asia. PLoS One 5: $\mathrm{e} 12575$.

Gardner, A.L., (Ed.). 2008. Mammals of South America. Vol. 1. Marsupials, xenarthrans, shrews, and bats. University of Chicago Press, Chicago, Ill.

Gene Codes Corporation. 2007. Sequencher version 4.8.Gene Codes Corp., Ann Arbor, Mich. 
Gifford, M.E., Powell, R., Larson, A., and Gutberlet, Jr., R.L. 2004. Population structure and history of a phenotypically variable teiid lizard (Ameiva chrysolaema) from Hispaniola: the influence of a geologically complex island. Mol. Phylogenet. Evol. 32: 735-748.

Graham, A. 2003. Geohistory models and Cenozoic paleoenvironments of the Caribbean region. Syst. Bot. 28: 378-386.

Hajibabaei, M., Singer, G.A.C., Hebert, P.D.N., and Hickey, D.A. 2007. DNA barcoding: how it complements taxonomy, molecular phylogenetics and population genetics. Trends in Genetics 23: 167-172.

Hebert, P.D.N., Cywinska, A., Ball, S.L., and deWaard, J.R. 2003. Biological identifications through DNA barcodes. Proc. R. Soc. Lond. B 270: 313-21.

Hoffman, F.G., and Baker, R.J. 2001. Systematics of bats of the genus Glossophaga (Chiroptera: Phyllostomidae) and phylogeography in G. soricina based on the cytochrome-b gene. J. Mammal. 82: 1092-101.

Iturralde-Vinent, M.A., and MacPhee, R.D.E. 1999. Paleogeography of the Caribbean region: implications for Cenozoic biogeography. Bull. Amer. Mus. Nat. Hist. 238: 1-95.

Kumar, S., and Gadagkar, S. R. 2000. Efficiency of the neighbour-joining method in reconstructing deep and shallow evolutionary relationships in large phylogenies. J. Mol. Evol. 51: 544-553.

Larsen, R.J., Knapp, M.C., Genoways, H.H., Khan, F.A.A., Larsen, P.A., Wilson, D.E., and Baker, R.J. 2012. Genetic diversity of Neotropical Myotis (Chiroptera: Vespertilionidae) 
with an emphasis on South American species. PLoS ONE 7(10): e46578.

doi:10.1371/journal.pone.0046578.

Lim, B.K. 2012. Preliminary assessment of neotropical mammal DNA barcodes: an underestimation of biodiversity. Open Zool. J. 5: 10-17.

Lim, B.K., and Arcila Hernandez, L.M. 2016. DNA barcoding of Jamaican bats: implications to Neotropical biodiversity. Mitochondrial DNA Part A 27: 3013-3019.

Lim, B.K., Loureiro, L.O., Upham, N.S., and Brocca, J.L. In press. Phylogeography of Dominican Republic bats and implications to systematic relationships in the Neotropics. J. Mammal.

Myers, N., Mittermeier, R.A., Mittermeier, C.G., da Fonseca, G.A.B., and Kent J. 2000. Biodiversity hotspots for conservation priorities. Nature 403(6772): 853-858.

Nei, M., and Kumar, S. 2000 Molecular evolution and phylogenetics. Oxford University Press, New York, NY.

Phillips, C.J., Pumo, D.E., Genoways, H.H., Ray, P.E., and Biskey, C.A. 1991. Mitochondrial DNA evolution and phylogeography in two Neotropical fruit bats, Artibeus jamaicensis and Artibeus lituratus. In Latin American mammalogy: history, biodiversity, and conservation. Edited by M.A. Mares and D.J. Schmidly. University of Oklahoma Press, Norman pp. $97-123$.

Presley, S.J., and Willig, M.R. 2008. Composition and structure of Caribbean bat (Chiroptera) assemblages: effects of inter-island distance, area, elevation and hurricane-induced disturbance. Global Ecol. Biogeogr. 17: 747-757. 
Reeder, D.M., Helgen, K.M., and Wilson, D.E. 2007. Global trends and biases in new mammal species discoveries. Occ. Pap. Mus. Texas Tech Univ. 269: 1-34.

Swanepoel, P., and Genoways, H.H. 1978. Revision of the Antillean bats of the genus Brachyphylla (Mammalia: Phyllostomatidae). Bull. Carnegie Mus. Nat. Hist. 12: 1-53.

Tamura, K., Stecher, G., Peterson, D., Filipski, A., and Kumar, S. 2013. MEGA6: molecular evolutionary genetics analysis version 6.0. Mol. Biol. Evol. 30: 2725-9.

Townsend, A.K., Rimmer, C.C., Latta, S.C., and Lovette, I.J. 2007. Ancient differentiation in the single-island avian radiation of endemic Hispaniolan chat-tanagers (Aves: Calyptophilus). Mol. Ecol. 16: 3634-3642. 


\section{Figure Captions}

Fig. 1. Maximum likelihood tree showing DNA barcode variation for 29 Caribbean bat species. Bootstrap support percentages $>50$ are reported from the maximum likelihood analysis first followed by support values from the neighbor-joining analysis.

Fig. 2. Maximum likelihood trees showing DNA barcode variation for 2 bat species (Eptesicus fuscus and Tadarida brasiliensis) with deep divergence between Caribbean (Dominican Republic in green) and Central and North American populations as well as bat species in the genus Myotis, including M. martiniquensis from Martinique (in red) in the Lesser Antilles with affinities to South American Myotis. Bootstrap support percentages $>50 \%$ are reported at the nodes. Average Kimura 2-parameter distances are reported between select pairs.

Fig. 3. Maximum likelihood trees showing DNA barcode variation for 3 bat genera endemic to the Caribbean (Jamaica in blue, Dominican Republic in green, and Martinique in red) as well as the widely distributed Molossus. Bootstrap support percentages $>50 \%$ are reported at the nodes. Average pairwise Kimura 2-parameter distances are reported between select pairs.

Fig. 4. Maximum likelihood trees showing DNA barcode variation of 3 bat species with a phylogeographic break between northern (purple N) and southern (orange S) Hispaniola. Bootstrap support percentages $>50 \%$ are reported at the nodes. Average Kimura 2-parameter distances are reported between select pairs. Darker gray-shading is lowland regions and lighter gray-shading is highland regions. 


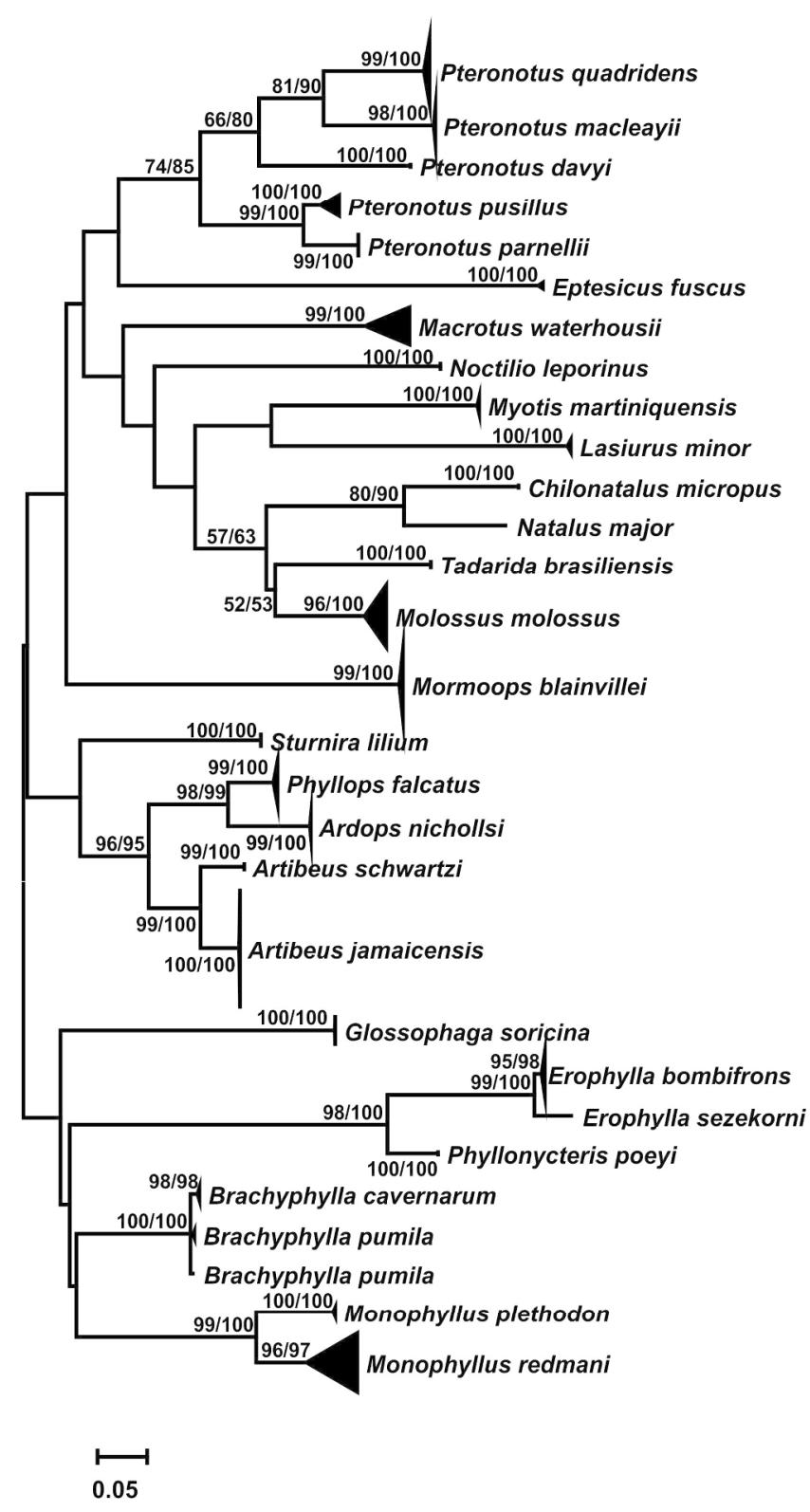

Maximum likelihood tree showing DNA barcode variation for 29 Caribbean bat species. Bootstrap support percentages $>65$ are reported from the maximum likelihood analysis first followed by support values from the neighbor-joining analysis.

Fig. 1

$141 \times 267 \mathrm{~mm}$ (300 x 300 DPI) 


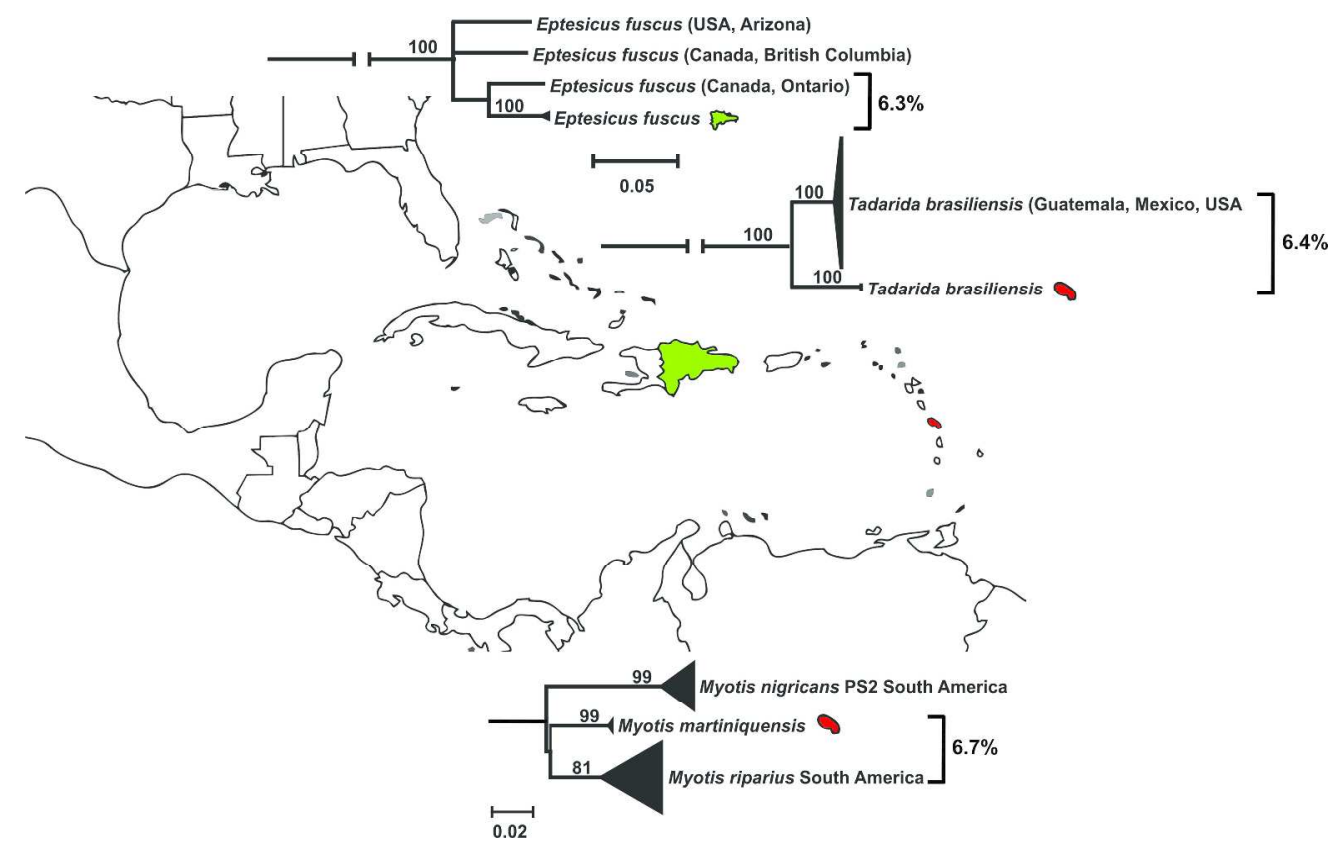

Maximum likelihood trees showing DNA barcode variation for 2 bat species (Eptesicus fuscus and Tadarida brasiliensis) with deep divergence between Caribbean (Dominican Republic in green) and Central and North American populations as well as bat species in the genus Myotis, including M. martiniquensis from Martinique (in red) in the Lesser Antilles with affinities to South American Myotis. Bootstrap support percentages $>50 \%$ are reported at the nodes. Average Kimura 2-parameter distances are reported between select pairs.

Fig. 2

$362 \times 228 \mathrm{~mm}(300 \times 300$ DPI $)$ 


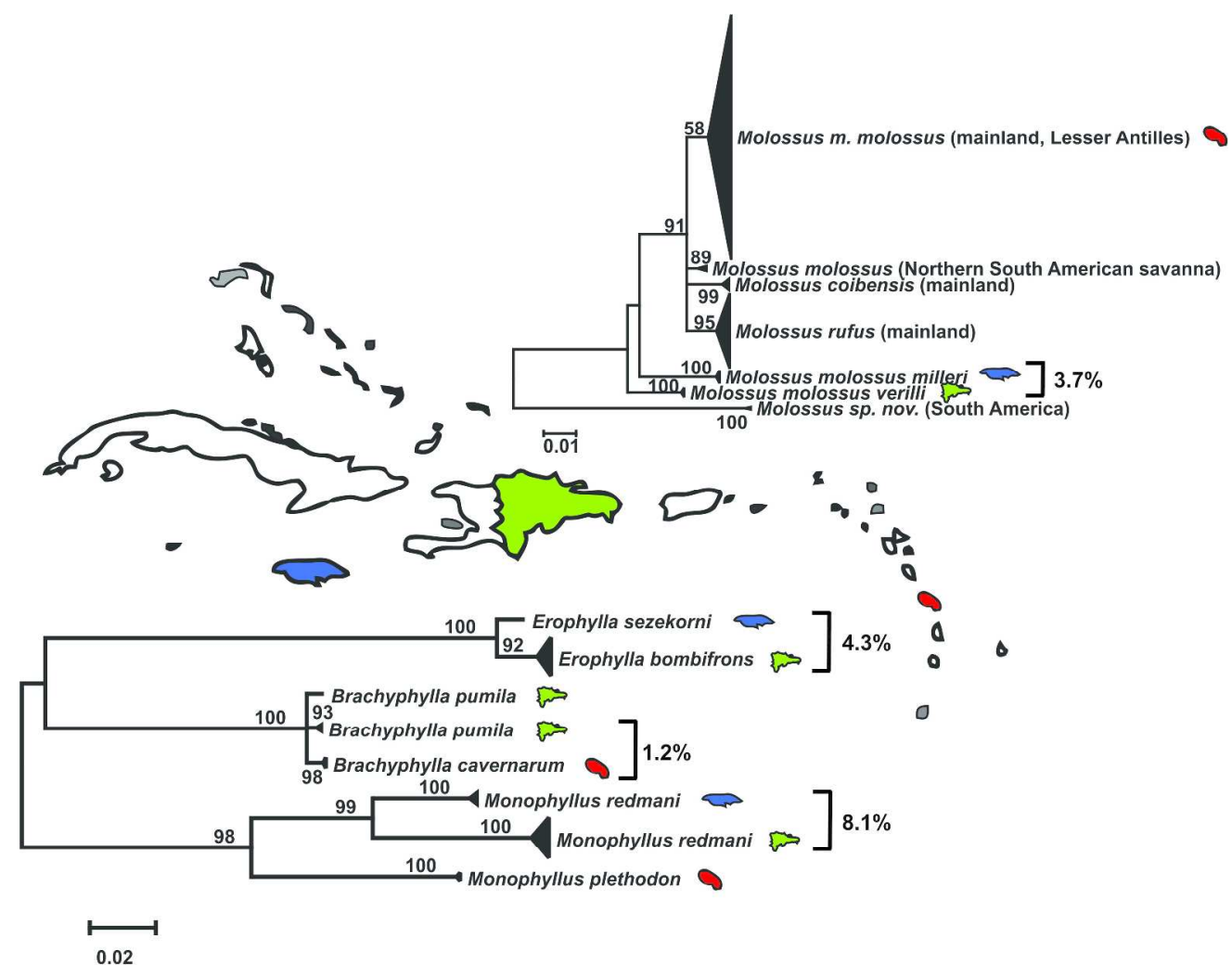

Maximum likelihood trees showing DNA barcode variation for 3 bat genera endemic to the Caribbean (Jamaica in blue, Dominican Republic in green, and Martinique in red) as well as the widely distributed Molossus. Bootstrap support percentages $>50 \%$ are reported at the nodes. Average pairwise Kimura 2parameter distances are reported between select pairs.

Fig. 3

$315 \times 248 \mathrm{~mm}(300 \times 300 \mathrm{DPI})$ 

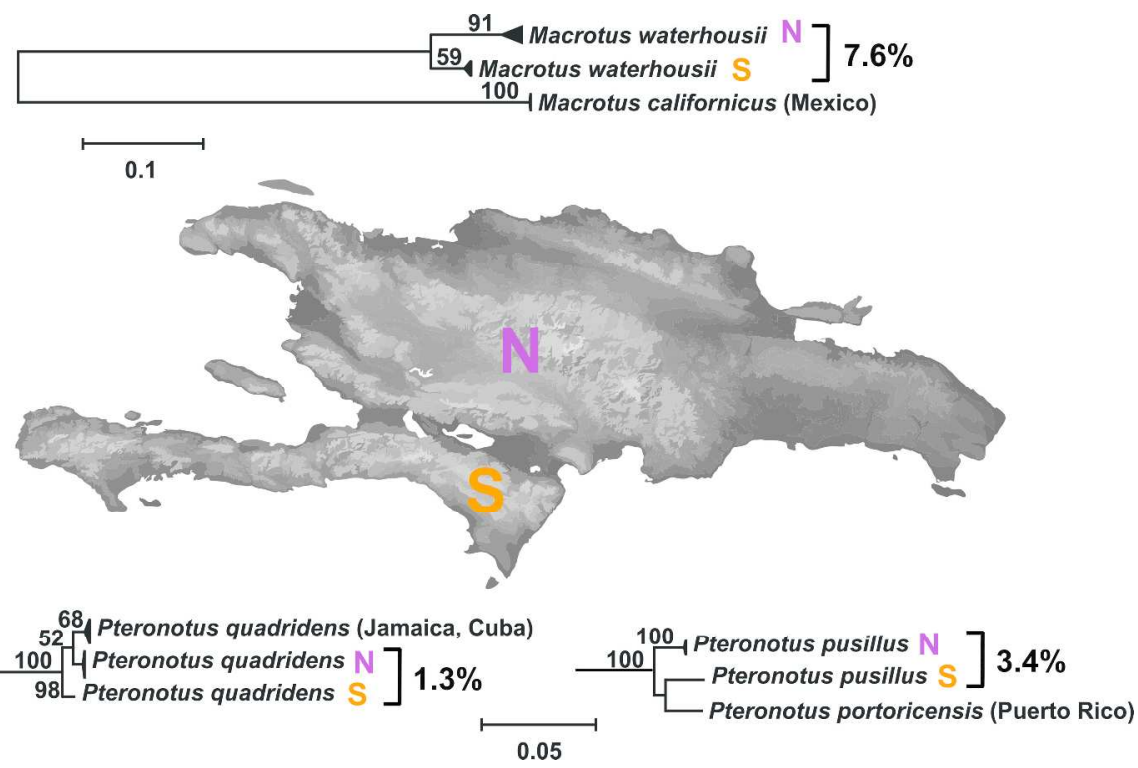

Maximum likelihood trees showing DNA barcode variation of 3 bat species with a phylogeographic break between northern (purple N) and southern (orange S) Hispaniola. Bootstrap support percentages $>50 \%$ are reported at the nodes. Average Kimura 2-parameter distances are reported between select pairs. Darker gray-shading is lowland regions and lighter gray-shading is highland regions.

Fig. 4

$357 \times 205 \mathrm{~mm}(300 \times 300 \mathrm{DPI})$ 
Table 1. Species of bats from Jamaica, Dominican Republic, and Martinique with sample sizes used in the molecular phylogenetic analysis of genetic variation based on DNA barcodes. If "0", the species occurs on the island but no sample was available in this study. An unfilled cell indicates that the species is not found on the island.

\begin{tabular}{|l|r|r|r|}
\hline Species & Jamaica & $\begin{array}{l}\text { Dominican } \\
\text { Republic }\end{array}$ & Martinique \\
\hline Ardops nichollsi & & & 6 \\
\hline Ariteus flavescens & 0 & & \\
\hline Artibeus jamaicensis & 12 & 26 & 3 \\
\hline Artibeus schwartzi & & & 4 \\
\hline Brachyphylla cavernarum & & & \\
\hline Brachyphylla pumila & & 3 & \\
\hline Chilonatalus micropus & 2 & 0 & \\
\hline Eptesicus fuscus & 0 & 2 & \\
\hline Erophylla bombifrons & & 14 & \\
\hline Erophylla sezekorni & 1 & & \\
\hline Eumops auripendulus & 0 & & \\
\hline Eumops glaucinus & 0 & & \\
\hline Glossophaga sorcina & 10 & & \\
\hline Lasiurus borealis & & & \\
\hline Lasiurus degelidus & 0 & & \\
\hline Macrotus waterhousii & 0 & & \\
\hline Molossus molossus & 11 & & \\
\hline Monophyllus redmani & 4 & & \\
\hline Monophyllus plethodon & & & \\
\hline Mormoops blainvillii & 13 & & \\
\hline Myotis martiniquensis & & & \\
\hline Natalus jamaicensis & 0 & & \\
\hline Natalus major & & & \\
\hline Natalus stramineus & & & \\
\hline
\end{tabular}




\begin{tabular}{|l|r|r|r|} 
Noctilio leporinus & 0 & 3 & 0 \\
\hline Nyctinomops macrotis & 0 & 0 & \\
\hline Phyllonycteris aphylla & 0 & & \\
\hline Phyllonycteris poeyi & & 2 & \\
\hline Phyllops falcatus & & 14 & \\
\hline Pteronotus davyi & & & 2 \\
\hline Pteronotus macleayii & 11 & & \\
\hline Pteronotus parnellii & 8 & & \\
\hline Pteronotus pusillus & & 7 & \\
\hline Pteronotus quadridens & 9 & 12 & \\
\hline Sturnira lilium & & & 5 \\
\hline Tadarida brasiliensis & 0 & 0 & 3 \\
\hline
\end{tabular}


Table 2. Sequence divergence based on Kimura 2-paramater model of nucleotide substitutions for bat diversity in the Caribbean. Species abbreviations in order of appearance in table are: Ardops nichollsi, Artibeus schwartzi, Artibeus jamaicensis, Brachyphylla cavernarum, Molossus molossus, Monophyllus plethodon, Myotis martiniquensis, Pteronotus davyii, Sturnira lilium, Tadarida brasiliensis, Brachphylla pumila, Eptesicus fuscus, Erophylla bombifrons, Lasiurus minor, Macrotus waterhousii, Monophyllus redmani, Mormoops blainvillei, Natalus major, Noctilio leporinus, Phyllonycteris poeyi, Phyllops falcatus, Pteronotus pusillus, Pteronotus quadridens, Chilonatalus micropus, Erophylla sezekorni, Glossophaga soricina, Pteronotus macleayii, Pteronotus parnellii, and Brachyphylla pumila.

\begin{tabular}{|c|c|c|c|c|c|c|c|c|c|c|}
\hline Ard_n & $\begin{array}{r}\text { Ard_n } \\
0.001\end{array}$ & Art_s & Art_j & B_c & Mol_m & Mon_p & Myo_m & Pt_d & S_1 & $T_{-} b$ \\
\hline Art_s & 0.149 & 0 & & & & & & & & \\
\hline Art_j & 0.150 & 0.071 & 0.0002 & & & & & & & \\
\hline B_c & 0.219 & 0.188 & 0.196 & 0.002 & & & & & & \\
\hline Mol_m & 0.238 & 0.215 & 0.233 & 0.224 & 0.022 & & & & & \\
\hline Mon_p & 0.250 & 0.238 & 0.242 & 0.206 & 0.257 & 0.002 & & & & \\
\hline Myo_m & 0.250 & 0.237 & 0.231 & 0.231 & 0.193 & 0.252 & 0.003 & & & \\
\hline Pt_d & 0.245 & 0.222 & 0.219 & 0.227 & 0.205 & 0.232 & 0.258 & 0 & & \\
\hline S_1 & 0.196 & 0.202 & 0.192 & 0.223 & 0.198 & 0.225 & 0.239 & 0.225 & 0 & \\
\hline$T_{-} b$ & 0.230 & 0.219 & 0.229 & 0.249 & 0.169 & 0.260 & 0.216 & 0.236 & 0.218 & \\
\hline B_p & 0.222 & 0.192 & 0.191 & 0.012 & 0.225 & 0.209 & 0.225 & 0.224 & 0.214 & 0.248 \\
\hline Ep_f & 0.254 & 0.260 & 0.235 & 0.271 & 0.245 & 0.269 & 0.259 & 0.272 & 0.261 & 0.240 \\
\hline Er_b & 0.275 & 0.264 & 0.254 & 0.255 & 0.278 & 0.249 & 0.245 & 0.273 & 0.262 & 0.283 \\
\hline L_m & 0.263 & 0.262 & 0.267 & 0.274 & 0.227 & 0.283 & 0.225 & 0.272 & 0.276 & 0.238 \\
\hline Ma_w & 0.237 & 0.217 & 0.212 & 0.226 & 0.234 & 0.251 & 0.223 & 0.255 & 0.227 & 0.235 \\
\hline Mon_r & 0.256 & 0.232 & 0.227 & 0.198 & 0.260 & 0.146 & 0.242 & 0.241 & 0.243 & 0.247 \\
\hline Mor_b & 0.264 & 0.234 & 0.226 & 0.238 & 0.257 & 0.268 & 0.252 & 0.236 & 0.228 & 0.260 \\
\hline Na_m & 0.254 & 0.256 & 0.252 & 0.253 & 0.200 & 0.262 & 0.242 & 0.237 & 0.246 & 0.184 \\
\hline No_1 & 0.249 & 0.229 & 0.236 & 0.226 & 0.229 & 0.236 & 0.227 & 0.248 & 0.214 & 0.237 \\
\hline Phn_p & 0.258 & 0.243 & 0.231 & 0.235 & 0.252 & 0.260 & 0.243 & 0.266 & 0.248 & 0.277 \\
\hline Php_f & 0.105 & 0.143 & 0.141 & 0.190 & 0.238 & 0.250 & 0.231 & 0.227 & 0.195 & 0.242 \\
\hline $\mathrm{Pt} \_\mathrm{pu}$ & 0.231 & 0.215 & 0.217 & 0.222 & 0.218 & 0.220 & 0.236 & 0.198 & 0.198 & 0.256 \\
\hline Pt_q & 0.255 & 0.233 & 0.255 & 0.241 & 0.231 & 0.228 & 0.255 & 0.175 & 0.235 & 0.242 \\
\hline C_m & 0.225 & 0.212 & 0.223 & 0.234 & 0.198 & 0.247 & 0.225 & 0.229 & 0.238 & 0.200 \\
\hline Er_s & 0.274 & 0.259 & 0.252 & 0.244 & 0.275 & 0.251 & 0.256 & 0.276 & 0.269 & 0.287 \\
\hline G_s & 0.237 & 0.197 & 0.208 & 0.219 & 0.226 & 0.241 & 0.226 & 0.275 & 0.260 & 0.235 \\
\hline Pt_m & 0.252 & 0.215 & 0.217 & 0.235 & 0.231 & 0.281 & 0.263 & 0.181 & 0.226 & 0.228 \\
\hline $\mathrm{Pt} \_\mathrm{pa}$ & 0.235 & 0.227 & 0.213 & 0.226 & 0.238 & 0.221 & 0.235 & 0.175 & 0.211 & 0.267 \\
\hline
\end{tabular}


Table 2. Continued

$\begin{array}{lccccccccr} & \text { B_p } & \text { Ep_f } & \text { Er_b } & \text { L_m } & \text { Ma_w } & \text { Mon_r } & \text { Mor_b } & \text { Na_m } & \text { No_1 } \\ \text { B_p } & 0.006 & & & & & & & & \\ \text { Ep_f } & 0.271 & 0.006 & & & & & & & \\ \text { Er_b } & 0.247 & 0.304 & 0.005 & & & & & & \\ \text { L_m } & 0.277 & 0.250 & 0.325 & 0.004 & & & & & \\ \text { Ma_w } & 0.220 & 0.245 & 0.283 & 0.237 & 0.047 & & & & \\ \text { Mon_r } & 0.203 & 0.274 & 0.265 & 0.253 & 0.243 & 0.034 & & & \\ \text { Mor_b } & 0.237 & 0.273 & 0.273 & 0.287 & 0.256 & 0.245 & 0.006 & & \\ \text { Na_m } & 0.250 & 0.265 & 0.309 & 0.244 & 0.233 & 0.262 & 0.266 & - & \\ \text { No_1 } & 0.226 & 0.285 & 0.263 & 0.252 & 0.233 & 0.256 & 0.240 & 0.243 & 0 \\ \text { Phn_p } & 0.239 & 0.298 & 0.149 & 0.327 & 0.276 & 0.267 & 0.295 & 0.291 & 0.271 \\ \text { Php_f } & 0.193 & 0.269 & 0.249 & 0.262 & 0.225 & 0.250 & 0.236 & 0.243 & 0.229 \\ \text { Pt_pu } & 0.225 & 0.239 & 0.272 & 0.254 & 0.229 & 0.242 & 0.221 & 0.253 & 0.217 \\ \text { Pt_q } & 0.236 & 0.277 & 0.272 & 0.275 & 0.238 & 0.247 & 0.233 & 0.253 & 0.236 \\ \text { C_m } & 0.221 & 0.262 & 0.281 & 0.248 & 0.236 & 0.266 & 0.261 & 0.151 & 0.222 \\ \text { Er_s } & 0.236 & 0.291 & 0.043 & 0.336 & 0.277 & 0.264 & 0.285 & 0.317 & 0.273 \\ \text { G_s } & 0.226 & 0.244 & 0.269 & 0.254 & 0.246 & 0.230 & 0.260 & 0.264 & 0.244 \\ \text { Pt_m } & 0.238 & 0.248 & 0.254 & 0.285 & 0.245 & 0.265 & 0.264 & 0.230 & 0.233 \\ \text { Pt_pa } & 0.228 & 0.245 & 0.263 & 0.266 & 0.232 & 0.230 & 0.222 & 0.238 & 0.217\end{array}$

Phn $\_$php_f $\quad$ Pt_pu Pt_q $\quad$ C_m $\quad$ Er_s $\quad$ G_s $\quad$ Pt_m $\quad$ Pt_pa Phn $\_$p $\quad 0$

$\begin{array}{lll}\text { Php_f } & 0.241 \quad 0.007\end{array}$

Pt_pu $\quad 0.279 \quad 0.237 \quad 0.010$

$\begin{array}{lllll}\text { Pt_q } & 0.284 & 0.251 & 0.196 & 0.007\end{array}$

C_m $\quad 0.292 \quad 0.225 \quad 0.246 \quad 0.250 \quad 0$

$\begin{array}{lllllll}\text { Er_s } & 0.162 & 0.266 & 0.276 & 0.281 & 0.290 & -\end{array}$

$\begin{array}{llllllll}\text { G_s } & 0.269 & 0.235 & 0.258 & 0.280 & 0.268 & 0.256 & 0.001\end{array}$

$\begin{array}{lllllllll}\text { Pt_m } & 0.262 & 0.229 & 0.201 & 0.156 & 0.228 & 0.252 & 0.259 & 0.003\end{array}$

$\begin{array}{llllllllll}\text { Pt_pa } & 0.290 & 0.223 & 0.078 & 0.203 & 0.243 & 0.269 & 0.270 & 0.208 & 0\end{array}$ 


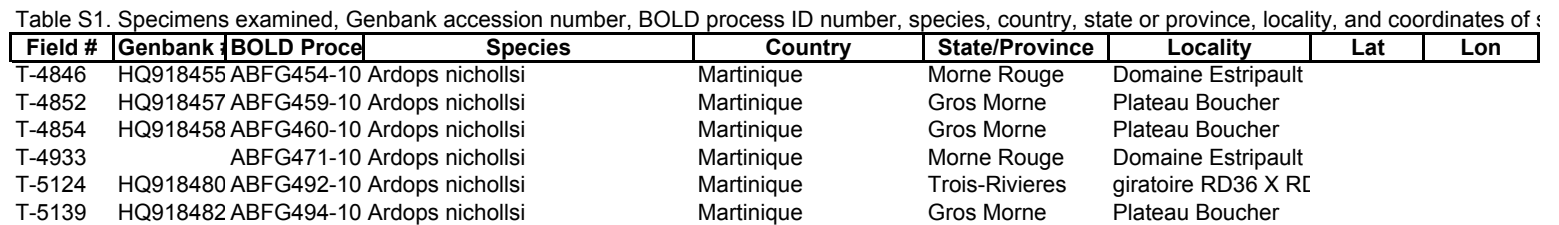

T-5139 HQ918482 ABFG494-10 Ardops nicholls JF447901 Artibeus amplus JF447908 Artibeus amplus EU160991 Artibeus fimbriatus JF446310 Artibeus fimbriatus EU160993 Artibeus fraterculus EU160994 Artibeus fraterculus F44048 JF446569 Artibeus jamaicensis F44061 JF446571 Artibeus jamaicensis F44062 JF446572 F44063 JF446570 F58954 KX354998 F58957 KX354999

F58958 KX355000 F58962 KX355001 F58963 KX355002 F58973 KX355003

F58974 KX355004 F58992 KX355005 F59224 KX355006 F59227 KX355007 F59242 KX355008 F59243 KX355009 F59259 KX355010 F59260 KX355011 F59268 KX355012 F59270 KX355013 F59274 KX355014 F59275 KX355015 F59276 KX355016 F59282 KX355017 F59283 KX355018 F59293 KX355019 F59294 KX355020 F59295 KX355021 F59297 KX355022 F59299 KX355023 F40075 F35412 JF446456

F35473 JF446457

F35475 JF446455

F35476 JF446458

F35500 JF446459

F35501 JF446460

F35502 JF446461

F35503 JF446462

F35504 JF446463

F35523 JF446454 F35524 JF446464 FN31390 JF446623 FN31485 JF446624 FN31486 JF446625 FN31487 JF446626 FN31488 JF446627 FN31810 JF446632 FN31811 JF446629 FN31812 JF446630 FN31813 JF446635

FN31814 JF446636 FN31828 JF446646 FN31829 JF446647 FN31830 JF446648 FN31880 JF446633 FN31935 JF446634 FN32307 JF446637

FN32308 JF446638

FN32309 JF446639

FN32310 JF446640

FN32311 JF446641

FN32312 JF446642

FN32400 JF446643

FN32401 JF446628

FN32402 JF446631

Artibeus jamaicensis Artibeus jamaicensis Artibeus jamaicensis Artibeus jamaicensis Artibeus jamaicensis Artibeus jamaicensis Artibeus jamaicensis Artibeus jamaicensis Artibeus jamaicensis Artibeus jamaicensis Artibeus jamaicensis Artibeus jamaicensis Artibeus jamaicensis Artibeus jamaicensis Artibeus jamaicensis Artibeus jamaicensis Artibeus jamaicensis Artibeus jamaicensis Artibeus jamaicensis Artibeus jamaicensis Artibeus jamaicensis Artibeus jamaicensis Artibeus jamaicensis Artibeus jamaicensis Artibeus jamaicensis Artibeus jamaicensis Artibeus jamaicensis Artibeus jamaicensis BCBN910-05 Artibeus jamaicensis Artibeus jamaicensis Artibeus jamaicensis Artibeus jamaicensis Artibeus jamaicensis Artibeus jamaicensis Artibeus jamaicensis Artibeus jamaicensis Artibeus jamaicensis Artibeus jamaicensis Artibeus jamaicensis Artibeus jamaicensis Artibeus jamaicensis Artibeus jamaicensis Artibeus jamaicensis Artibeus jamaicensis Artibeus jamaicensis Artibeus jamaicensis Artibeus jamaicensis Artibeus jamaicensis Artibeus jamaicensis Artibeus jamaicensis Artibeus jamaicensis Artibeus jamaicensis Artibeus jamaicensis Artibeus jamaicensis Artibeus jamaicensis Artibeus jamaicensis Artibeus jamaicensis Artibeus jamaicensis Artibeus jamaicensis Artibeus jamaicensis Artibeus jamaicensis Artibeus jamaicensis Artibeus jamaicensis Artibeus jamaicensis

\begin{tabular}{|c|c|c|c|}
\hline Costa Rica & Limon & Cano Palma Biologi 10.6 & -83.533 \\
\hline Costa Rica & Limon & Cano Palma Biologi 10.6 & -83.533 \\
\hline Costa Rica & Limon & Cano Palma Biologi 10.6 & -83.533 \\
\hline Costa Rica & Limon & Cano Palma Biologi 10.6 & -83.533 \\
\hline Dominican Republic & National District & Santo Domingo, Jar 18.49399 & -69.9561 \\
\hline Dominican Republic & Pedernales & $9 \mathrm{~km} \mathrm{NE}$ of Pederne 18.08317 & -71.6709 \\
\hline Dominican Republic & Pedernales & $9 \mathrm{~km} \mathrm{NE}$ of Pederne 18.08317 & -71.6709 \\
\hline Dominican Republic & Pedernales & $15 \mathrm{~km} \mathrm{~N}$ of Pederna 18.16949 & -71.7652 \\
\hline Dominican Republic & Pedernales & $15 \mathrm{~km} \mathrm{~N}$ of Pederna 18.16949 & -71.7652 \\
\hline Dominican Republic & Pedernales & $1 \mathrm{~km}$ SE of Aguas $\wedge 18.16282$ & -71.6905 \\
\hline Dominican Republic & Pedernales & $1 \mathrm{~km}$ SE of Aguas $\wedge 18.16282$ & -71.6905 \\
\hline Dominican Republic & Barahona & Cueva de los Patos 17.96002 & -71.1833 \\
\hline Dominican Republic & Sanchez Ramirez & Cueva Honda de Ju 19.13234 & -70.0796 \\
\hline Dominican Republic & Monte Plata & Cueva la Chepa, 1418.86914 & -69.5763 \\
\hline Dominican Republic & El Seibo & Miches, Hotel Coco 18.98384 & -69.0371 \\
\hline Dominican Republic & El Seibo & Miches, Hotel Coco 18.98384 & -69.0371 \\
\hline Dominican Republic & El Seibo & $10 \mathrm{~km} \mathrm{NE}$ of Miches 19.02935 & -68.9488 \\
\hline Dominican Republic & El Seibo & $10 \mathrm{~km}$ NE of Miches 19.02935 & -68.9488 \\
\hline Dominican Republic & La Altagracia & Laguna Bavaro $\quad 18.64782$ & -68.364 \\
\hline Dominican Republic & La Altagracia & Laguna Bavaro & -68.364 \\
\hline Dominican Republic & La Altagracia & Cueva Taina 3km S 18.76605 & -68.5482 \\
\hline Dominican Republic & La Altagracia & Cueva Taina 3km S 18.76605 & -68.5482 \\
\hline Dominican Republic & La Altagracia & Cueva Taina 3km S 18.76605 & -68.5482 \\
\hline Dominican Republic & La Altagracia & Barcelo Bavaro Res 18.65971 & -68.3942 \\
\hline Dominican Republic & La Altagracia & Barcelo Bavaro Res 18.65971 & -68.3942 \\
\hline Dominican Republic & La Altagracia & Barcelo Bavaro Res 18.65277 & -68.4001 \\
\hline Dominican Republic & La Altagracia & Barcelo Bavaro Res 18.65277 & -68.4001 \\
\hline Dominican Republic & La Altagracia & Barcelo Bavaro Res 18.65277 & -68.4001 \\
\hline Dominican Republic & La Altagracia & Laguna Yauya, Res 18.50922 & -68.3742 \\
\hline Dominican Republic & La Altagracia & Laguna Yauya, Res 18.50922 & -68.3742 \\
\hline Ecuador & Esmeraldas & $2 \mathrm{Km}$ S Of Alto Tam 0.9 & -78.55 \\
\hline El Salvador & Ahuachapan & El Imposible, San F/ 13.85 & -90 \\
\hline El Salvador & Ahuachapan & El Imposible, El Ref 13.8 & -90 \\
\hline El Salvador & Ahuachapan & El Imposible, El Ref 13.8 & -90 \\
\hline El Salvador & Ahuachapan & El Imposible, El Ref 13.8 & -90 \\
\hline El Salvador & Ahuachapan & El Imposible, El Ref 13.8 & -90 \\
\hline El Salvador & Ahuachapan & El Imposible, El Ref 13.8 & -90 \\
\hline El Salvador & Ahuachapan & El Imposible, El Ref 13.8 & -90 \\
\hline El Salvador & Ahuachapan & El Imposible, El Ref 13.8 & -90 \\
\hline El Salvador & Ahuachapan & El Imposible, El Ref 13.8 & -90 \\
\hline El Salvador & Ahuachapan & El Imposible, El Ref 13.8 & -90 \\
\hline El Salvador & Ahuachapan & El Imposible, El Ref 13.8 & -90 \\
\hline Guatemala & El Progreso & $10 \mathrm{Km} \mathrm{Nw}$ Of El Ral 14.917 & -90.1 \\
\hline Guatemala & El Progreso & Rio Uyus, 5 Km E C 14.95 & -89.833 \\
\hline Guatemala & El Progreso & Rio Uyus, 5 Km E C 14.95 & -89.833 \\
\hline Guatemala & El Progreso & Rio Uyus, $5 \mathrm{Km}$ E C 14.95 & -89.833 \\
\hline Guatemala & El Progreso & Rio Uyus, $5 \mathrm{Km} \mathrm{E} \mathrm{C} 14.95$ & -89.833 \\
\hline Guatemala & Peten & $1.5 \mathrm{Km} \mathrm{S}, 1 \mathrm{Km} \mathrm{W}(16.3$ & -89.3333 \\
\hline Guatemala & Peten & $1.5 \mathrm{Km} \mathrm{S}, 1 \mathrm{Km} \mathrm{W}(16.3$ & -89.333 \\
\hline Guatemala & Peten & $1.5 \mathrm{Km} \mathrm{S}, 1 \mathrm{Km} \mathrm{W}(16.3$ & -89.333 \\
\hline Guatemala & Peten & $1.5 \mathrm{Km} \mathrm{S}, 1 \mathrm{Km} \mathrm{W}(16.3$ & -89.333 \\
\hline Guatemala & Peten & $1.5 \mathrm{Km} \mathrm{S}, 1 \mathrm{Km} \mathrm{W}(16.3$ & -89.333 \\
\hline Guatemala & Peten & Tikal $\quad 17.2$ & -89.617 \\
\hline Guatemala & Peten & Tikal & -89.617 \\
\hline Guatemala & Peten & Tikal & -89.617 \\
\hline Guatemala & Peten & Tikal & -89.617 \\
\hline Guatemala & Peten & $10 \mathrm{Km} \mathrm{N}$ Of Tikal 17.3 & -89.617 \\
\hline Guatemala & Peten & Campo Los Guacan 17.6 & -90.817 \\
\hline Guatemala & Peten & Campo Los Guacan 17.6 & -90.817 \\
\hline Guatemala & Peten & Campo Los Guacan 17.6 & -90.817 \\
\hline Guatemala & Peten & Campo Los Guacan 17.6 & -90.817 \\
\hline Guatemala & Peten & Campo Los Guacan 17.6 & -90.817 \\
\hline Guatemala & Peten & Campo Los Guacan 17.6 & -90.817 \\
\hline Guatemala & Peten & Biotope Cerro Cahu 17 & -89.733 \\
\hline Guatemala & Peten & Biotope Cerro Cahu 17 & -89.733 \\
\hline Guatemala & Peten & Biotope Cerro Cahu 17 & -89.733 \\
\hline
\end{tabular}




\begin{tabular}{|c|c|c|c|c|c|c|}
\hline FN32403 & JF446644 & Artibeus jamaicensis & Guatemala & Peten & Biotope Cerro Cahu 17 & -89.733 \\
\hline FN32404 & JF446645 & Artibeus jamaicensis & Guatemala & Peten & Biotope Cerro Cahu 17 & -89.733 \\
\hline F53479 & KT023180 & Artibeus jamaicensis & JAMAICA & SAINT CATHEF & IST. CLAIR CAVE, E 18.15056 & -77.0743 \\
\hline F53480 & КT023181 & Artibeus jamaicensis & JAMAICA & SAINT CATHEF & I ST. CLAIR CAVE, E 18.15056 & -77.0743 \\
\hline F53481 & KT023182 & Artibeus jamaicensis & JAMAICA & SAINT ELIZAB & T 1 KM NE OF BALAC 18.18281 & -77.6437 \\
\hline F53482 & KT023183 & Artibeus jamaicensis & JAMAICA & SAINT ELIZAB & T 1 KM NE OF BALA( 18.18281 & -77.6437 \\
\hline F53501 & KT023184 & Artibeus jamaicensis & JAMAICA & WESTMORELA & JIBLUEFIELDS BEAC 18.17472 & -78.0315 \\
\hline F53502 & KT023185 & Artibeus jamaicensis & JAMAICA & WESTMORELA & JIBLUEFIELDS BEAC 18.17472 & -78.0315 \\
\hline F53507 & КT023186 & Artibeus jamaicensis & JAMAICA & WESTMORELA & Jl 13 KM NW OF BLU 18.28515 & -78.0549 \\
\hline F53511 & KT023187 & Artibeus jamaicensis & JAMAICA & WESTMORELA & J 13.5 KM NW OF BL 18.29126 & -78.0438 \\
\hline F53514 & KT023188 & Artibeus jamaicensis & JAMAICA & SAINT ANN & 1 KM N OF QUEEN 18.44363 & -77.4068 \\
\hline F53516 & КT023189 & Artibeus jamaicensis & JAMAICA & SAINT ANN & 4 KM NE OF QUEE 18.46348 & -77.3892 \\
\hline F53518 & КT023190 & Artibeus jamaicensis & JAMAICA & PORTLAND & SHERWOOD FORE 18.14076 & -76.3761 \\
\hline F53524 & КT023191 & Artibeus jamaicensis & JAMAICA & PORTLAND & 2 KM SW OF SHEF 18.13228 & -76.3861 \\
\hline $\mathrm{T}-5123$ & HQ918479 ABFG491-10 & Artibeus jamaicensis & Martinique & Trois-Rivieres & giratoire RD36 X R & \\
\hline V-2015: N & HQ918452 & Artibeus jamaicensis & Martinique & & Habitation Ceron 14.83 & -61.2203 \\
\hline V-2016: N & HQ918453 & Artibeus jamaicensis & Martinique & & Habitation Ceron & -61.2203 \\
\hline V-2021: N & HQ918454 & Artibeus jamaicensis & Martinique & & Case Petit & -61.2064 \\
\hline V-2223 & HQ918479 & Artibeus jamaicensis & Martinique & & giratoire RD36 X R[ & \\
\hline ASK379 & JF447040 & Artibeus jamaicensis & Mexico & Campeche & Ciudad Del Carmen & \\
\hline AVB0803: & HM208645 & Artibeus jamaicensis & Mexico & Campeche & Vicinity of Zoh-Lagu 18.5887 & -89.4179 \\
\hline AVB0803: & HM208646 & Artibeus jamaicensis & Mexico & Campeche & Vicinity of Zoh-Lagu 18.5971 & -89.4222 \\
\hline AVB0803: & HM208647 & Artibeus jamaicensis & Mexico & Campeche & Vicinity of Zoh-Lagu 18.5971 & -89.4222 \\
\hline FN29408 & JF447039 & Artibeus jamaicensis & Mexico & Campeche & $44 \mathrm{Km}$ S Of Constitı 18.25 & -90.067 \\
\hline FN29991 & JF447037 & Artibeus jamaicensis & Mexico & Quintana Roo & $1 \mathrm{Km} \mathrm{S}, 10 \mathrm{Km} \mathrm{W} \mathrm{C} 20.85$ & -86.917 \\
\hline FN29992 & JF447038 & Artibeus jamaicensis & Mexico & Quintana Roo & $1 \mathrm{Km} \mathrm{S}, 10 \mathrm{Km} \mathrm{W} \mathrm{C} 20.85$ & -86.917 \\
\hline FN30115 & JF447060 & Artibeus jamaicensis & Mexico & Campeche & 3.7 Km Se Of Chekı 18.8 & -90.983 \\
\hline FN30168 & JF447061 & Artibeus jamaicensis & Mexico & Campeche & $3.7 \mathrm{Km} \mathrm{Se}$ Of Chekı 18.8 & -90.983 \\
\hline FN30169 & JF447062 & Artibeus jamaicensis & Mexico & Campeche & $3.7 \mathrm{Km}$ Se Of Chekı 18.8 & -90.983 \\
\hline FN30170 & JF447063 & Artibeus jamaicensis & Mexico & Campeche & $3.7 \mathrm{Km}$ Se Of Chekı 18.8 & -90.983 \\
\hline FN30171 & JF447064 & Artibeus jamaicensis & Mexico & Campeche & 3.7 Km Se Of Chekı 18.8 & -90.983 \\
\hline FN30172 & JF447065 & Artibeus jamaicensis & Mexico & Campeche & $3.7 \mathrm{Km} \mathrm{Se}$ Of Chekı 18.8 & -90.983 \\
\hline FN30188 & ABMXC273-C & (Artibeus jamaicensis & Mexico & Campeche & $3.7 \mathrm{Km} \mathrm{Se}$ Of Chekı 18.8 & -90.983 \\
\hline FN30470 & JF447042 & Artibeus jamaicensis & Mexico & Yucatan & $15 \mathrm{Km}$ S Of Tekax, & \\
\hline FN30477 & JF447043 & Artibeus jamaicensis & Mexico & Campeche & $60 \mathrm{Km}$ Se Of Dzibal 19.167 & -89.333 \\
\hline FN30478 & JF447044 & Artibeus jamaicensis & Mexico & Campeche & $60 \mathrm{Km}$ Se Of Dzibal 19.167 & -89.333 \\
\hline FN30481 & JF447045 & Artibeus jamaicensis & Mexico & Campeche & $60 \mathrm{Km}$ Se Of Dzibal 19.167 & -89.333 \\
\hline FN30482 & JF447046 & Artibeus jamaicensis & Mexico & Campeche & $60 \mathrm{Km}$ Se Of Dzibal 19.167 & -89.333 \\
\hline FN30483 & JF447047 & Artibeus jamaicensis & Mexico & Campeche & $60 \mathrm{Km}$ Se Of Dzibal 19.167 & -89.333 \\
\hline FN30484 & JF447048 & Artibeus jamaicensis & Mexico & Campeche & $60 \mathrm{Km}$ Se Of Dzibal 19.167 & -89.333 \\
\hline FN30527 & JF447049 & Artibeus jamaicensis & Mexico & Campeche & $60 \mathrm{Km}$ Se Of Dzibal 19.167 & -89.333 \\
\hline FN30611 & JF447944 & Artibeus jamaicensis & Mexico & Campeche & $18 \mathrm{Km}$ S Of X-Kanh 18.917 & -89.333 \\
\hline FN30615 & JF447050 & Artibeus jamaicensis & Mexico & Campeche & $18 \mathrm{Km}$ S Of X-Kanh 18.917 & -89.333 \\
\hline FN30616 & JF447051 & Artibeus jamaicensis & Mexico & Campeche & $18 \mathrm{Km}$ S Of X-Kanh 18.917 & -89.333 \\
\hline FN30617 & JF447052 & Artibeus jamaicensis & Mexico & Campeche & $18 \mathrm{Km}$ S Of X-Kanh 18.917 & -89.333 \\
\hline FN30618 & JF445312 & Artibeus jamaicensis & Mexico & Campeche & $18 \mathrm{Km}$ S Of X-Kanh 18.917 & -89.333 \\
\hline FN30619 & JF447053 & Artibeus jamaicensis & Mexico & Campeche & $18 \mathrm{Km}$ S Of X-Kanh 18.917 & -89.333 \\
\hline FN30620 & JF447054 & Artibeus jamaicensis & Mexico & Campeche & $18 \mathrm{Km}$ S Of X-Kanh 18.917 & -89.333 \\
\hline FN30621 & JF447055 & Artibeus jamaicensis & Mexico & Campeche & $18 \mathrm{Km} \mathrm{S}$ Of X-Kanh 18.917 & -89.333 \\
\hline FN30622 & JF447056 & Artibeus jamaicensis & Mexico & Campeche & $18 \mathrm{Km} \mathrm{S}$ Of X-Kanh 18.917 & -89.333 \\
\hline FN30623 & JF445313 & Artibeus jamaicensis & Mexico & Campeche & $18 \mathrm{Km}$ S Of X-Kanh 18.917 & -89.333 \\
\hline FN30892 & JF447066 & Artibeus jamaicensis & Mexico & Quintana Roo & $6 \mathrm{Km} \mathrm{S}$ Of Majahua 18.683 & -87.733 \\
\hline FN30895 & JF447067 & Artibeus jamaicensis & Mexico & Quintana Roo & $6 \mathrm{Km} \mathrm{S}$ Of Majahua 18.683 & -87.733 \\
\hline FN30899 & JF447068 & Artibeus jamaicensis & Mexico & Quintana Roo & $6 \mathrm{Km} \mathrm{S}$ Of Majahua 18.683 & -87.733 \\
\hline FN30900 & JF447069 & Artibeus jamaicensis & Mexico & Quintana Roo & $6 \mathrm{Km} \mathrm{S}$ Of Majahua 18.683 & -87.733 \\
\hline FN30940 & JF447070 & Artibeus jamaicensis & Mexico & Quintana Roo & Laguna Noh-Bec, 219.133 & -88.183 \\
\hline FN30941 & JF447071 & Artibeus jamaicensis & Mexico & Quintana Roo & Laguna Noh-Bec, 219.133 & -88.183 \\
\hline FN30942 & JF447072 & Artibeus jamaicensis & Mexico & Quintana Roo & Laguna Noh-Bec, 219.133 & -88.183 \\
\hline FN30943 & JF447073 & Artibeus jamaicensis & Mexico & Quintana Roo & Laguna Noh-Bec, 219.133 & -88.183 \\
\hline FN30944 & JF447074 & Artibeus jamaicensis & Mexico & Quintana Roo & Laguna Noh-Bec, 219.133 & -88.183 \\
\hline FN30956 & JF447945 & Artibeus jamaicensis & Mexico & Quintana Roo & Laguna Noh-Bec, 219.133 & -88.183 \\
\hline FN30957 & JF447059 & Artibeus jamaicensis & Mexico & Quintana Roo & Laguna Noh-Bec, 219.133 & -88.183 \\
\hline FN30958 & JF447057 & Artibeus jamaicensis & Mexico & Quintana Roo & Laguna Noh-Bec, 219.133 & -88.183 \\
\hline FN30961 & JF447058 & Artibeus jamaicensis & Mexico & Quintana Roo & Laguna Noh-Bec, 219.133 & -88.183 \\
\hline FN32775 & JF447041 & Artibeus jamaicensis & Mexico & Campeche & El Remata, $14 \mathrm{Km} \mathrm{V}$ & \\
\hline F48079 & JF459374 & Artibeus jamaicensis & Nicaragua & & & \\
\hline F48080 & JF459375 & Artibeus jamaicensis & Nicaragua & & & \\
\hline F48110 & JF459376 & Artibeus jamaicensis & Nicaragua & & & \\
\hline F48111 & JF459373 & Artibeus jamaicensis & Nicaragua & & & \\
\hline F38036 & JF447342 & Artibeus jamaicensis & Panama & Canal Zone & Gamboa & -79.7 \\
\hline
\end{tabular}




\begin{tabular}{|c|c|c|c|c|c|c|c|}
\hline F38054 & JF447341 & Artibeus jamaicensis & Panama & Canal Zone & Gamboa & 9.1 & -79.7 \\
\hline F38089 & JF447349 & Artibeus jamaicensis & Panama & & Parque Nacional Alt 8 & 8.683 & -79.933 \\
\hline F38090 & JF447348 & Artibeus jamaicensis & Panama & & Parque Nacional Alt 8 & 8.683 & -79.933 \\
\hline F38091 & JF447350 & Artibeus jamaicensis & Panama & & Parque Nacional Alt 8 & 8.683 & -79.933 \\
\hline F38168 & JF447346 & Artibeus jamaicensis & Panama & Chiriqui & Santa Clara & 8.833 & -82.75 \\
\hline F38179 & JF447347 & Artibeus jamaicensis & Panama & Chiriqui & Santa Clara & 8.833 & -82.75 \\
\hline F38188 & \multicolumn{2}{|c|}{ ABSCA104-0 Artibeus jamaicensis } & Panama & Darien & \multicolumn{2}{|c|}{ Estacion Pirre, Parc 8} & -77.717 \\
\hline F38189 & JF447343 & Artibeus jamaicensis & Panama & Darien & \multicolumn{2}{|l|}{ Estacion Pirre, Parc 8} & -77.717 \\
\hline F38221 & JF447344 & Artibeus jamaicensis & Panama & Darien & \multicolumn{2}{|l|}{ Estacion Pirre, Parc 8} & -77.717 \\
\hline F38222 & JF447345 & Artibeus jamaicensis & Panama & Darien & \multicolumn{2}{|l|}{ Estacion Pirre, Parc 8} & -77.717 \\
\hline & AF061340 & Artibeus jamaicensis & & & & & \\
\hline & NC_002009 & Artibeus jamaicensis & & & & & \\
\hline T-4836 & HQ918452 ABFG445-10 & Artibeus jamaicensis/schwartzi & Martinique & Le Precheur & Habitation Ceron & 14.83 & -61.2203 \\
\hline $\mathrm{T}-4837$ & \multicolumn{2}{|c|}{ HQ918453 ABFG446-10 Artibeus jamaicensis/schwartzi } & Martinique & Le Precheur & Habitation Ceron & 14.83 & -61.2203 \\
\hline \multirow[t]{7}{*}{ T-4842 } & HQ918454 ABFG450-10 & Artibeus jamaicensis/schwartzi & Martinique & Le Precheur & Case Petit & 14.8189 & -61.2064 \\
\hline & JQ600004 & Artibeus lituratus & Guyana & & $5 \mathrm{Km}$ Se Of Surame & 4.1 & -59.05 \\
\hline & JQ601182 & Artibeus lituratus & Suriname & & Kutari River Camp & 2.17526 & -56.788 \\
\hline & JQ601298 & Artibeus obscurus & Suriname & & Brownsberg Nature & 4.917 & -55.167 \\
\hline & JQ601324 & Artibeus obscurus & Suriname & & Tafelberg, Base Cal & 3.848 & -56.19 \\
\hline & JQ601270 & Artibeus planirostris & Suriname & & Sipaliwini River Can & 2.289 & -56.607 \\
\hline & JQ601282 & Artibeus planirostris & Suriname & & Brownsberg Nature & 4.933 & -55.2 \\
\hline NC_0224 & NC_02242 CYTC7862-1 & Brachyphylla cavernarum & Martinique & & & & \\
\hline $\mathrm{T}-5 \overline{2} 38$ & HQ918488 ABFG502-10 & Brachyphylla cavernarum & Martinique & Trois llets & village-poterie & & \\
\hline $\mathrm{T}-5240$ & ABFG504-10 & Brachyphylla cavernarum & Martinique & Trois llets & village-poterie & & \\
\hline $\mathrm{T}-5241$ & HQ918489 ABFG505-10 & Brachyphylla cavernarum & Martinique & Trois llets & village-poterie & & \\
\hline F58989 & KX355024 & Brachyphylla pumila & Dominican Republic & Barahona & Cueva de los Patos & 17.96002 & -71.1833 \\
\hline F58990 & KX355025 & Brachyphylla pumila & Dominican Republic & Barahona & Cueva de los Patos & 17.96002 & -71.1833 \\
\hline F58991 & KX355026 & Brachyphylla pumila & Dominican Republic & Barahona & Cueva de los Patos & 17.96002 & -71.1833 \\
\hline F53466 & KT023192 & Chilonatalus micropus & JAMAICA & SAINT CATHERII & ST. CLAIR CAVE, $\leqq$ & 18.15101 & -77.0909 \\
\hline F53467 & KT023193 & Chilonatalus micropus & JAMAICA & SAINT CATHER & ST. CLAIR CAVE, $\subseteq$ & 18.15101 & -77.0909 \\
\hline F58967 & KX355027 & Eptesicus fuscus & Dominican Republic & Pedernales & $1 \mathrm{~km}$ SE of Aguas $\Lambda$ & 18.16282 & -71.6905 \\
\hline F59205 & KX355028 & Eptesicus fuscus & Dominican Republic & La Vega & Parque Nacional Ar & 19.0632 & -70.8642 \\
\hline F58972 & KX355029 & Erophylla bombifrons & Dominican Republic & Pedernales & $1 \mathrm{~km}$ SE of Aguas $\Lambda$ & 18.16282 & -71.6905 \\
\hline F58986 & KX355030 & Erophylla bombifrons & Dominican Republic & Barahona & Cueva de los Patos & 17.96002 & -71.1833 \\
\hline F58987 & KX355031 & Erophylla bombifrons & Dominican Republic & Barahona & Cueva de los Patos & 17.96002 & -71.1833 \\
\hline F58988 & KX355032 & Erophylla bombifrons & Dominican Republic & Barahona & Cueva de los Patos & 17.96002 & -71.1833 \\
\hline F58994 & KX355033 & Erophylla bombifrons & Dominican Republic & & Cueva del Pomier & 18.46698 & -70.1358 \\
\hline F58995 & KX355034 & Erophylla bombifrons & Dominican Republic & & Cueva del Pomier & 18.46698 & -70.1358 \\
\hline F58996 & KX355035 & Erophylla bombifrons & Dominican Republic & & Cueva del Pomier & 18.46698 & -70.1358 \\
\hline F59218 & KX355036 & Erophylla bombifrons & Dominican Republic & Sanchez Ramirez & Cueva Honda de Ju & 19.13234 & -70.0796 \\
\hline F59219 & KX355037 & Erophylla bombifrons & Dominican Republic & Sanchez Ramirez & Cueva Honda de Ju & 19.13234 & -70.0796 \\
\hline F59220 & KX355038 & Erophylla bombifrons & Dominican Republic & Sanchez Ramirez & Cueva Honda de Ju & 19.13234 & -70.0796 \\
\hline F59271 & KX355039 & Erophylla bombifrons & Dominican Republic & La Altagracia & Laguna Bavaro & 18.64782 & -68.364 \\
\hline F59288 & KX355040 & Erophylla bombifrons & Dominican Republic & La Altagracia & Barcelo Bavaro Res & 18.65277 & -68.4001 \\
\hline F59289 & KX355041 & Erophylla bombifrons & Dominican Republic & La Altagracia & Barcelo Bavaro Res & 18.65277 & -68.4001 \\
\hline F59305 & KX355042 & Erophylla bombifrons & Dominican Republic & La Altagracia & Punta Cana Ecologi & 18.52531 & -68.3808 \\
\hline F53494 & KT023194 & Erophylla sezekorni & JAMAICA & SAINT ELIZABET & 4 KM NE OF BALAC & 18.20687 & -77.6258 \\
\hline F44002 & JF446596 & Glossophaga commissarisi & Costa Rica & Limon & Tortuga Lodge, Tort 1 & 10.567 & -83.517 \\
\hline F44003 & JF459387 & Glossophaga commissarisi & Costa Rica & Limon & Tortuga Lodge, Tort 1 & 10.567 & -83.517 \\
\hline F44025 & JF446597 & Glossophaga commissarisi & Costa Rica & Limon & Cano Palma Biologi 1 & 10.6 & -83.533 \\
\hline F44026 & JF448034 & Glossophaga commissarisi & Costa Rica & Limon & Cano Palma Biologi 1 & 10.6 & -83.533 \\
\hline $\mathrm{F} 44045$ & JF446598 & Glossophaga commissarisi & Costa Rica & Limon & Cano Palma Biologi 1 & 10.6 & -83.533 \\
\hline F44059 & JF448036 & Glossophaga commissarisi & Costa Rica & Limon & Cano Palma Biologi 1 & 10.6 & -83.533 \\
\hline F44060 & JF448035 & Glossophaga commissarisi & Costa Rica & Limon & Cano Palma Biologi 1 & 10.6 & -83.533 \\
\hline F35408 & JF446500 & Glossophaga commissarisi & El Salvador & Ahuachapan & El Imposible, San F/ 1 & 13.85 & -90 \\
\hline F35409 & JF446512 & Glossophaga commissarisi & El Salvador & Ahuachapan & El Imposible, San F/ 1 & 13.85 & -90 \\
\hline F35436 & JF446509 & Glossophaga commissarisi & El Salvador & Ahuachapan & El Imposible, San F/1 & 13.85 & -90 \\
\hline F35437 & JF446508 & Glossophaga commissarisi & El Salvador & Ahuachapan & El Imposible, San F/1 & 13.85 & -90 \\
\hline F35438 & JF446507 & Glossophaga commissarisi & El Salvador & Ahuachapan & El Imposible, San F/1 & 13.85 & -90 \\
\hline F35439 & JF446501 & Glossophaga commissarisi & El Salvador & Ahuachapan & El Imposible, San F/1 & 13.85 & -90 \\
\hline F35466 & JF446502 & Glossophaga commissarisi & El Salvador & Ahuachapan & El Imposible, El Ref 1 & 13.8 & -90 \\
\hline F35467 & JF446503 & Glossophaga commissarisi & El Salvador & Ahuachapan & El Imposible, El Ref 1 & 13.8 & -90 \\
\hline F35468 & JF446513 & Glossophaga commissarisi & El Salvador & Ahuachapan & El Imposible, El Ref 1 & 13.8 & -90 \\
\hline F35469 & JF446511 & Glossophaga commissarisi & El Salvador & Ahuachapan & El Imposible, El Ref 1 & 13.8 & -90 \\
\hline F35470 & JF446510 & Glossophaga commissarisi & El Salvador & Ahuachapan & El Imposible, El Ref 1 & 13.8 & -90 \\
\hline F35509 & JF446504 & Glossophaga commissarisi & El Salvador & Ahuachapan & El Imposible, El Ref 1 & 13.8 & -90 \\
\hline F35510 & JF446506 & Glossophaga commissarisi & El Salvador & Ahuachapan & El Imposible, El Ref 1 & 13.8 & -90 \\
\hline F35512 & $\mathrm{JF} 446505$ & Glossophaga commissarisi & El Salvador & Ahuachapan & El Imposible, El Ref 1 & 13.8 & -90 \\
\hline FN31936 & JF446782 & Glossophaga commissarisi & Guatemala & Peten & $10 \mathrm{Km} \mathrm{N}$ Of Tikal & 17.3 & -89.617 \\
\hline FN31937 & JF446785 & Glossophaga commissarisi & Guatemala & Peten & $10 \mathrm{Km} \mathrm{N} \mathrm{Of} \mathrm{Tikal}$ & 17.3 & -89.617 \\
\hline FN31938 & JF446784 & Glossophaga commissarisi & Guatemala & Peten & $10 \mathrm{Km} \mathrm{N}$ Of Tikal & 17.3 & -89.617 \\
\hline FN31972 & JF446783 & Glossophaga commissarisi & Guatemala & Peten & $10 \mathrm{Km} \mathrm{N}$ Of Tikal & 17.3 & -89.617 \\
\hline FN31973 & $\mathrm{JF} 446781$ & Glossophaga commissarisi & Guatemala & Peten & $10 \mathrm{Km} \mathrm{N}$ Of Tikal & 17.3 & -89.617 \\
\hline FN31974 & JF446779 & Glossophaga commissarisi & Guatemala & Peten & $10 \mathrm{Km} N$ Of Tikal & 17.3 & -89.617 \\
\hline FN31975 & $\mathrm{JF} 446780$ & Glossophaga commissarisi & Guatemala & Peten & $10 \mathrm{Km} \mathrm{N}$ Of Tikal & 17.3 & -89.617 \\
\hline FN32320 & JF446778 & Glossophaga commissarisi & Guatemala & Peten & Campo Los Guacan 1 & 17.6 & -90.817 \\
\hline FN33102 & JF447256 & Glossophaga commissarisi & Mexico & Chiapas & $18.5 \mathrm{Km} \mathrm{S}$ Of Front 1 & 15.517 & -92.117 \\
\hline
\end{tabular}


FN33103 JF447257 FN33104 JF447255 FN33110 JF447254 F38066 JF447412 F38099 JF447411 F38126 JF447413 F38183 JF447410 F35513 JF448037 F34024 JF446788 F34025 JF446789 F34026 JF446787 F34027 JF446786 FN31381 JF446792 FN31382 JF446793 FN31383 JF446790 FN31384 JF446791

F51452 JF454668 F51482 JF454664 F51483 JF454660 F51492 JF454666

F51535 JF454663 F51536 JF459162 F51551 JF454659 F51552 JF454669

F51574 JF454662 F51755 JF454661 F51805 JF454665 F51830 JF454667 F51853 JF454670 FN33257 JF454671 FN33258 JF454673

FN33259 JF454674 FN33260 JF454675 FN33261 JF454676 FN33262 JF454658 FN33265 JF454672 FN33266 EF080359 F42873 JF447819 F42875 JF447807

F42876 JF448039 F42934 JF447813 F42982 JF447808 F42983 JF447818 F42984 JF447817 F42985 JF447805 F42988 JF447816 F42989 JF447815 F43040 JF447814 F43084 JF448038 F43127 JF447812

F43131 JF447804 F43133 JF447811 F43134 JF447806 F43138 JF447810

F43139 JF447809 HQ625002

F44467 JF435476

F37140 JF435484

F37324 JF448043 F40343 JF448042 F40548 JF435483

F54204 JF448847 FN31468 JF435374 FN31469 JF448044 FN31762 JF435359 FN31763 JF435360 FN31764 JF435361 FN31765 JF435362 FN31766 JF435363 FN31767 JF435364 FN31768 JF435365 FN31786 JF446796 FN31787 JF435366 FN31788 JF435471 FN31840 JF435372 FN31976 JF435469 FN32249 JF435470 FN32250 JF435468 FN32251 JF435373
Glossophaga commissarisi Glossophaga commissarisi Glossophaga commissarisi Glossophaga commissarisi Glossophaga commissaris Glossophaga commissarisi Glossophaga commissarisi Glossophaga leachii Glossophaga leachii Glossophaga leachii Glossophaga leachii Glossophaga leachii Glossophaga leachii Glossophaga leachii Glossophaga leachii Glossophaga leachii Glossophaga longirostris Glossophaga longirostris Glossophaga longirostris Glossophaga longirostris Glossophaga longirostris Glossophaga longirostris Glossophaga longirostris Glossophaga longirostris Glossophaga longirostris Glossophaga longirostris Glossophaga longirostris Glossophaga longirostris Glossophaga longirostris Glossophaga longirostris Glossophaga longirostris Glossophaga longirostris Glossophaga longirostris Glossophaga longirostris Glossophaga longirostris Glossophaga longirostris Glossophaga longirostris Glossophaga longirostris Glossophaga longirostris Glossophaga longirostris Glossophaga longirostris Glossophaga longirostris Glossophaga longirostris Glossophaga longirostris Glossophaga longirostris Glossophaga longirostris Glossophaga longirostris Glossophaga longirostris Glossophaga longirostris Glossophaga longirostris Glossophaga longirostris Glossophaga longirostris Glossophaga longirostris Glossophaga longirostris Glossophaga longirostris Glossophaga soricina Glossophaga soricina Glossophaga soricina Glossophaga soricina Glossophaga soricina Glossophaga soricina Glossophaga soricina Glossophaga soricina Glossophaga soricina Glossophaga soricina Glossophaga soricina Glossophaga soricina Glossophaga soricina Glossophaga soricina Glossophaga soricina Glossophaga soricina Glossophaga soricina Glossophaga soricina Glossophaga soricina Glossophaga soricina Glossophaga soricina Glossophaga soricina Glossophaga soricina Glossophaga soricina Glossophaga soricina

\begin{tabular}{|c|c|c|c|}
\hline Mexico & Chiapas & $18.5 \mathrm{Km}$ S Of Frontı 15.517 & -92.117 \\
\hline Mexico & Chiapas & $18.5 \mathrm{Km} \mathrm{S}$ Of Front 15.517 & -92.117 \\
\hline Mexico & Chiapas & $18.5 \mathrm{Km} \mathrm{S}$ Of Front 15.517 & -92.117 \\
\hline Panama & & Parque Nacional Alt 8.683 & -79.933 \\
\hline Panama & & Parque Nacional Alt 8.683 & -79.933 \\
\hline Panama & & Parque Nacional Alt 8.683 & -79.933 \\
\hline Panama & Chiriqui & Santa Clara $\quad 8.833$ & -82.75 \\
\hline El Salvador & Ahuachapan & El Imposible, El Ref 13.8 & -90 \\
\hline Guatemala & El Progreso & Rio Uyus, $5 \mathrm{Km} \mathrm{E} \mathrm{C} 14.95$ & -89.833 \\
\hline Guatemala & El Progreso & Rio Uyus, $5 \mathrm{Km} \mathrm{E} \mathrm{C} 14.95$ & -89.833 \\
\hline Guatemala & El Progreso & Rio Uyus, $5 \mathrm{Km} \mathrm{E} \mathrm{C} 14.95$ & -89.833 \\
\hline Guatemala & El Progreso & Rio Uyus, $5 \mathrm{Km} \mathrm{E} \mathrm{C} 14.95$ & -89.833 \\
\hline Guatemala & El Progreso & $10 \mathrm{Km} \mathrm{Nw}$ Of El Ral 14.9167 & -90.1 \\
\hline Guatemala & El Progreso & $10 \mathrm{Km} \mathrm{Nw}$ Of El Ral 14.917 & -90.1 \\
\hline Guatemala & El Progreso & $10 \mathrm{Km} \mathrm{Nw}$ Of El Ral 14.9167 & -90.1 \\
\hline Guatemala & El Progreso & $10 \mathrm{Km} \mathrm{Nw}$ Of El Ral 14.9167 & -90.1 \\
\hline Guyana & Upper Takut & Dadanawa Ranch 2.824 & -59.526 \\
\hline Guyana & Upper Takut & Dadanawa Ranch & -59.526 \\
\hline Guyana & Upper Takut & Dadanawa Ranch & -59.526 \\
\hline Guyana & Upper Takut & Dadanawa Ranch & -59.526 \\
\hline Guyana & Upper Takut & Dadanawa Ranch & -59.526 \\
\hline Guyana & Upper Takut & Dadanawa Ranch & -59.526 \\
\hline Guyana & Upper Takut & Dadanawa Ranch & -59.526 \\
\hline Guyana & Upper Takut & Dadanawa Ranch & -59.526 \\
\hline Guyana & Upper Takut & Dadanawa Ranch & -59.526 \\
\hline Guyana & Upper Takut & $1 \mathrm{Km}$ Upstream Fro 2.486 & -59.476 \\
\hline Guyana & Upper Takut & $1 \mathrm{Km}$ Upstream Fro 2.486 & -59.476 \\
\hline Guyana & Upper Takut & $1 \mathrm{Km}$ Upstream Fro 2.486 & -59.476 \\
\hline Guyana & Upper Takut & $1 \mathrm{Km}$ Upstream Fro 2.486 & -59.476 \\
\hline Guyana & Upper Takut & Lethem $\quad 3.383$ & -59.8 \\
\hline Guyana & Upper Takut & Lethem & -59.8 \\
\hline Guyana & Upper Takut & Lethem & -59.8 \\
\hline Guyana & Upper Takut & Lethem & -59.8 \\
\hline Guyana & Upper Takut & Lethem & -59.8 \\
\hline Guyana & Upper Takut & Lethem & -59.8 \\
\hline Guyana & Upper Takut & Lethem & -59.8 \\
\hline Guyana & Upper Takut & Lethem $\quad 3.38333$ & -59.8 \\
\hline Venezuela & & Pozon, 50 Km Ne C 6.05 & -67.417 \\
\hline Venezuela & & Pozon, $50 \mathrm{Km} \mathrm{Ne} \mathrm{C} 6.05$ & -67.417 \\
\hline Venezuela & Amazonas & Pozon, $50 \mathrm{Km} \mathrm{Ne} \mathrm{C} 6.05$ & -67.417 \\
\hline Venezuela & & Pozon, $50 \mathrm{Km} \mathrm{Ne} \mathrm{C} 6.05$ & -67.417 \\
\hline Venezuela & & Pozon, $50 \mathrm{Km} \mathrm{Ne} \mathrm{C} 6.05$ & -67.417 \\
\hline Venezuela & & Pozon, $50 \mathrm{Km} \mathrm{Ne} \mathrm{C} 6.05$ & -67.417 \\
\hline Venezuela & & Pozon, 50 Km Ne C 6.05 & -67.417 \\
\hline Venezuela & Amazonas & Pozon, $50 \mathrm{Km} \mathrm{Ne} \mathrm{C} 6.05$ & -67.417 \\
\hline Venezuela & & Pozon, $50 \mathrm{Km} \mathrm{Ne} \mathrm{C} 6.05$ & -67.417 \\
\hline Venezuela & & Pozon, $50 \mathrm{Km} \mathrm{Ne} \mathrm{C} 6.05$ & -67.417 \\
\hline Venezuela & & Pozon, $50 \mathrm{Km} \mathrm{Ne} \mathrm{C} 6.05$ & -67.417 \\
\hline Venezuela & Bolivar & Hato La Florida, 357.567 & -65.867 \\
\hline Venezuela & Guarico & Rio Orituco, $10 \mathrm{Km} 9.4$ & -66.467 \\
\hline Venezuela & Guarico & Rio Orituco, $10 \mathrm{Km} 9.4$ & -66.467 \\
\hline Venezuela & Guarico & Rio Orituco, $10 \mathrm{Km} 9.4$ & -66.467 \\
\hline Venezuela & Guarico & Rio Orituco, $10 \mathrm{Km} 9.4$ & -66.467 \\
\hline Venezuela & Guarico & Rio Orituco, $10 \mathrm{Km} 9.4$ & -66.467 \\
\hline Venezuela & Guarico & Rio Orituco, $10 \mathrm{Km} 9.4$ & -66.467 \\
\hline Brazil & Sao Paulo & Caetetus Ecological-22.383 & -49.667 \\
\hline Ecuador & Napo & Parque Nacional Ya-0.683 & -76.433 \\
\hline Ecuador & Napo & Parque Nacional Ya -0.783 & -76.433 \\
\hline Ecuador & Napo & Parque Nacional Ya & \\
\hline Ecuador & Napo & Parque Nacional Ya -0.65 & -76.45 \\
\hline Ecuador & & Onkone Gare, $38 \mathrm{KI}-0.657$ & -76.454 \\
\hline Guatemala & El Progreso & Rio Uyus, $5 \mathrm{Km} \mathrm{E} \mathrm{C} 14.95$ & -89.833 \\
\hline Guatemala & El Progreso & Rio Uyus, $5 \mathrm{Km} \mathrm{E} \mathrm{C} 14.95$ & -89.8333 \\
\hline Guatemala & Peten & $1.5 \mathrm{Km} \mathrm{S}, 1 \mathrm{Km} \mathrm{W}(16.3$ & -89.333 \\
\hline Guatemala & Peten & $1.5 \mathrm{Km} \mathrm{S}, 1 \mathrm{Km} \mathrm{W}(16.3$ & -89.333 \\
\hline Guatemala & Peten & $1.5 \mathrm{Km} \mathrm{S}, 1 \mathrm{Km} \mathrm{W}(16.3$ & -89.333 \\
\hline Guatemala & Peten & $1.5 \mathrm{Km} \mathrm{S}, 1 \mathrm{Km} \mathrm{W}(16.3$ & -89.333 \\
\hline Guatemala & Peten & $1.5 \mathrm{Km} \mathrm{S}, 1 \mathrm{Km} \mathrm{W}(16.3$ & -89.333 \\
\hline Guatemala & Peten & $1.5 \mathrm{Km} \mathrm{S}, 1 \mathrm{Km} \mathrm{W}(16.3$ & -89.333 \\
\hline Guatemala & Peten & $1.5 \mathrm{Km} \mathrm{S}, 1 \mathrm{Km} \mathrm{W}(16.3$ & -89.333 \\
\hline Guatemala & Peten & $1.5 \mathrm{Km} \mathrm{S}, 1 \mathrm{Km} \mathrm{W}(16.3$ & -89.3333 \\
\hline Guatemala & Peten & $1.5 \mathrm{Km} \mathrm{S}, 1 \mathrm{Km} \mathrm{W}(16.3$ & -89.333 \\
\hline Guatemala & Peten & $1.5 \mathrm{Km} \mathrm{S}, 1 \mathrm{Km} \mathrm{W}(16.3$ & -89.3333 \\
\hline Guatemala & Peten & $\begin{array}{ll}\text { Tikal } & 17.2\end{array}$ & -89.617 \\
\hline Guatemala & Peten & $10 \mathrm{Km} N$ Of Tikal $\quad 17.3$ & -89.617 \\
\hline Guatemala & Peten & Biotope Cerro Cahu 17 & -89.733 \\
\hline Guatemala & Peten & Biotope Cerro Cahu 17 & -89.733 \\
\hline Guatemala & Peten & Biotope Cerro Cahu 17 & -89.733 \\
\hline Guatemala & Peten & El Remate $\quad 17$ & -89.717 \\
\hline
\end{tabular}




\begin{tabular}{|c|c|c|c|c|c|c|}
\hline FN32282 & JF446794 & Glossophaga soricina & Guatemala & Peten & El Remate $\quad 17$ & -89.717 \\
\hline FN32313 & JF435368 & Glossophaga soricina & Guatemala & Peten & Campo Los Guacan 17.6 & -90.817 \\
\hline FN32319 & JF435369 & Glossophaga soricina & Guatemala & Peten & Campo Los Guacan 17.6 & -90.817 \\
\hline FN32321 & JF435370 & Glossophaga soricina & Guatemala & Peten & Campo Los Guacan 17.6 & -90.817 \\
\hline FN32322 & JF435371 & Glossophaga soricina & Guatemala & Peten & Campo Los Guacan 17.6 & -90.817 \\
\hline FN32323 & $J F 446795$ & Glossophaga soricina & Guatemala & Peten & Campo Los Guacan 17.6 & -90.817 \\
\hline F34419 & JF435457 & Glossophaga soricina & Guyana & Demerara-Mahaic & c Georgetown $\quad 6.817$ & -58.167 \\
\hline F34420 & JF435458 & Glossophaga soricina & Guyana & Demerara-Mahaic & c Georgetown & -58.167 \\
\hline F34421 & JF435459 & Glossophaga soricina & Guyana & Demerara-Mahaic & c Georgetown & -58.167 \\
\hline F34422 & JF435460 & Glossophaga soricina & Guyana & Demerara-Mahaic & c Georgetown & -58.167 \\
\hline F34423 & JF435461 & Glossophaga soricina & Guyana & Demerara-Mahaic & c Georgetown & -58.167 \\
\hline F34424 & JF435462 & Glossophaga soricina & Guyana & Demerara-Mahaic & c Georgetown & -58.167 \\
\hline F34425 & JF435463 & Glossophaga soricina & Guyana & Demerara-Mahaic & c Georgetown $\quad 6.817$ & -58.167 \\
\hline F34465 & JF435464 & Glossophaga soricina & Guyana & East Berbice-Core & $€$ Orealla Creek, Cor $€ 5.333$ & -57.333 \\
\hline F34808 & JF435465 & Glossophaga soricina & Guyana & Barima-Waini & Baramita, Old Worlc 7.367 & -60.483 \\
\hline F34914 & JF435466 & Glossophaga soricina & Guyana & Barima-Waini & Baramita, Old Worlc 7.367 & -60.483 \\
\hline F35107 & JF435354 & Glossophaga soricina & Guyana & Barima-Waini & Backdam, Reserve 7.35 & -60.45 \\
\hline F36370 & JF435355 & Glossophaga soricina & Guyana & Upper Takutu-Upr & $5 \mathrm{Km} \mathrm{Se}$ Of Suram 4.1 & -59.05 \\
\hline F36721 & JF435340 & Glossophaga soricina & Guyana & Upper Takutu-Upr & $5 \mathrm{Km}$ Se Of Suram 4.1 & -59.05 \\
\hline F36722 & JF435341 & Glossophaga soricina & Guyana & Upper Takutu-Upr & $5 \mathrm{Km}$ Se Of Surame 4.1 & -59.05 \\
\hline F36828 & JF435342 & Glossophaga soricina & Guyana & Upper Demerara-I & Tropenbos, $20 \mathrm{Km} \leqslant 5.15$ & -58.7 \\
\hline F36920 & JF435343 & Glossophaga soricina & Guyana & Upper Demerara-I & Tropenbos, $20 \mathrm{Km} \leqslant 5.15$ & -58.7 \\
\hline F36921 & JF435344 & Glossophaga soricina & Guyana & Upper Demerara-I & Tropenbos, $20 \mathrm{Km} \leqslant 5.15$ & -58.7 \\
\hline F36922 & JF435345 & Glossophaga soricina & Guyana & Upper Demerara-I & Tropenbos, $20 \mathrm{Km} \leqslant 5.15$ & -58.7 \\
\hline F36923 & JF435346 & Glossophaga soricina & Guyana & Upper Demerara-I & Tropenbos, $20 \mathrm{Km} \leqslant 5.15$ & -58.7 \\
\hline F36924 & JF435347 & Glossophaga soricina & Guyana & Upper Demerara-I & Tropenbos, $20 \mathrm{Km} \leqslant 5.15$ & -58.7 \\
\hline F36947 & JF435348 & Glossophaga soricina & Guyana & Upper Demerara-I & Tropenbos, $20 \mathrm{Km} \leqslant 5.15$ & -58.7 \\
\hline F36970 & JF435349 & Glossophaga soricina & Guyana & Upper Demerara-I & Tropenbos, $20 \mathrm{Km} \leqslant 5.15$ & -58.7 \\
\hline F36971 & JF435350 & Glossophaga soricina & Guyana & Upper Demerara-I & Tropenbos, $20 \mathrm{Km} \leqslant 5.15$ & -58.7 \\
\hline F36986 & JF435351 & Glossophaga soricina & Guyana & Upper Demerara-I & Tropenbos, $20 \mathrm{Km} \leqslant 5.15$ & -58.7 \\
\hline F36987 & JF435352 & Glossophaga soricina & Guyana & Upper Demerara-I & Tropenbos, $20 \mathrm{Km} \leqslant 5.15$ & -58.7 \\
\hline F36991 & JF435353 & Glossophaga soricina & Guyana & Upper Demerara-I & Tropenbos, $20 \mathrm{Km} \leqslant 5.15$ & -58.7 \\
\hline F38639 & JF435435 & Glossophaga soricina & Guyana & Upper Takutu-Upr & Chodikar River, 55 |1.367 & -58.767 \\
\hline F38939 & JF435436 & Glossophaga soricina & Guyana & Potaro-Siparuni & Iwokrama Reserve, 4.75 & -59.017 \\
\hline F38969 & JF435437 & Glossophaga soricina & Guyana & Potaro-Siparuni & Iwokrama Reserve, 4.75 & -59.017 \\
\hline F39146 & JF435375 & Glossophaga soricina & Guyana & Potaro-Siparuni & Iwokrama Reserve, 4.633 & -58.717 \\
\hline F39281 & JF435438 & Glossophaga soricina & Guyana & Potaro-Siparuni & Iwokrama Reserve, 4.733 & -58.85 \\
\hline F39433 & JF435439 & Glossophaga soricina & Guyana & Potaro-Siparuni & Iwokrama Reserve, 4.733 & -58.85 \\
\hline F39436 & JF435433 & Glossophaga soricina & Guyana & Potaro-Siparuni & Iwokrama Reserve, 4.733 & -58.85 \\
\hline F39437 & JF435434 & Glossophaga soricina & Guyana & Potaro-Siparuni & Iwokrama Reserve, 4.733 & -58.85 \\
\hline F39580 & JF435440 & Glossophaga soricina & Guyana & Potaro-Siparuni & Iwokrama Reserve, 4.733 & -58.85 \\
\hline F39581 & JF435441 & Glossophaga soricina & Guyana & Potaro-Siparuni & Iwokrama Reserve, 4.733 & -58.85 \\
\hline F39582 & JF435442 & Glossophaga soricina & Guyana & Potaro-Siparuni & Iwokrama Reserve, 4.733 & -58.85 \\
\hline F39668 & JF435443 & Glossophaga soricina & Guyana & Potaro-Siparuni & Iwokrama Reserve, 4.667 & -58.85 \\
\hline F39750 & JF435444 & Glossophaga soricina & Guyana & Cuyuni-Mazaruni & Kuwaima Fall, Mazc 6.05 & -60.65 \\
\hline F39777 & EF080360 & Glossophaga soricina & Guyana & Cuyuni-Mazaruni & Kuwaima Fall, Mazє 6.05 & -60.65 \\
\hline F40647 & & ABGYD688-C Glossophaga soricina & Guyana & Potaro-Siparuni & 'S' Falls, Siparuni Ri 4.533 & -59.083 \\
\hline F40677 & & ABGYD716-C Glossophaga soricina & Guyana & Potaro-Siparuni & 'S' Falls, Siparuni Ri 4.533 & -59.083 \\
\hline F40678 & JF459171 & Glossophaga soricina & Guyana & Potaro-Siparuni & 'S' Falls, Siparuni Ri 4.533 & -59.083 \\
\hline F40709 & JF459170 & Glossophaga soricina & Guyana & Potaro-Siparuni & 'S' Falls, Siparuni Ri 4.533 & -59.083 \\
\hline F40732 & JF459169 & Glossophaga soricina & Guyana & Potaro-Siparuni & 'S' Falls, Siparuni Ri 4.533 & -59.083 \\
\hline F40863 & JF459168 & Glossophaga soricina & Guyana & Potaro-Siparuni & Pakatau Falls, Sipaı 4.75 & -59.017 \\
\hline F43229 & EF080362 & Glossophaga soricina & Guyana & Cuyuni-Mazaruni & Namai Creek, $5 \mathrm{Km} 5.8$ & -61.1 \\
\hline F43326 & EF080363 & Glossophaga soricina & Guyana & Cuyuni-Mazaruni & $\begin{array}{ll}\text { Paruima } & 5.817\end{array}$ & -61.067 \\
\hline F43339 & JF435427 & Glossophaga soricina & Guyana & Potaro-Siparuni & 38 Mile Camp, 35 K 4.367 & -58.85 \\
\hline $\mathrm{F} 43643$ & JF435429 & Glossophaga soricina & Guyana & Potaro-Siparuni & Cow Fly Camp, 40 r 4.333 & -58.817 \\
\hline F43662 & JF435430 & Glossophaga soricina & Guyana & Potaro-Siparuni & Cow Fly Camp, 40 + 4.333 & -58.817 \\
\hline F43706 & JF435431 & Glossophaga soricina & Guyana & Potaro-Siparuni & Cow Fly Camp, 40 + 4.333 & -58.817 \\
\hline F43764 & JF435432 & Glossophaga soricina & Guyana & Potaro-Siparuni & Gorge Camp, $40 \mathrm{Kn} 4.333$ & -58.8 \\
\hline F43894 & JF435428 & Glossophaga soricina & Guyana & Potaro-Siparuni & Turtle Mountain, 104.733 & -58.717 \\
\hline F44684 & JF435411 & Glossophaga soricina & Guyana & Potaro-Siparuni & Kabukalli Landing, | 4.283 & -58.517 \\
\hline F44695 & JF435412 & Glossophaga soricina & Guyana & Potaro-Siparuni & Kabukalli Landing, | 4.283 & -58.517 \\
\hline F44723 & JF435414 & Glossophaga soricina & Guyana & Potaro-Siparuni & Kabukalli Landing, | 4.283 & -58.517 \\
\hline F44766 & JF435415 & Glossophaga soricina & Guyana & Potaro-Siparuni & Kabukalli Landing, I 4.283 & -58.517 \\
\hline F44821 & JF435416 & Glossophaga soricina & Guyana & Potaro-Siparuni & Kabukalli Landing, I 4.283 & -58.517 \\
\hline F45110 & JF435417 & Glossophaga soricina & Guyana & Potaro-Siparuni & Iwokrama Field Stat 4.667 & -58.683 \\
\hline F45172 & JF435413 & Glossophaga soricina & Guyana & Potaro-Siparuni & Iwokrama Field Stat 4.667 & -58.683 \\
\hline F45215 & JF459167 & Glossophaga soricina & Guyana & Potaro-Siparuni & Three Mile Camp, I 4.633 & -58.717 \\
\hline F45217 & JF435418 & Glossophaga soricina & Guyana & Potaro-Siparuni & Three Mile Camp, I 4.633 & -58.717 \\
\hline F45218 & JF435419 & Glossophaga soricina & Guyana & Potaro-Siparuni & Three Mile Camp, I 4.633 & -58.717 \\
\hline F45225 & JF435420 & Glossophaga soricina & Guyana & Potaro-Siparuni & Three Mile Camp, I 4.633 & -58.717 \\
\hline F45226 & JF435421 & Glossophaga soricina & Guyana & Potaro-Siparuni & Three Mile Camp, I 4.633 & -58.717 \\
\hline F45334 & JF435422 & Glossophaga soricina & Guyana & Demerara-Mahaic & c Ceiba Biological Stz 6.5 & -58.217 \\
\hline F45392 & JF435423 & Glossophaga soricina & Guyana & Demerara-Mahaic & c Georgetown, Botani 6.817 & -58.117 \\
\hline F50355 & JF435424 & Glossophaga soricina & Guyana & Upper Demerara-I & West Pibiri, Mabura 5.033 & -58.633 \\
\hline F50356 & JF435425 & Glossophaga soricina & Guyana & Upper Demerara-I & West Pibiri, Mabura 5.033 & -58.633 \\
\hline F50424 & JF435426 & Glossophaga soricina & Guyana & Upper Demerara-I & West Pibiri, Mabura 5.033 & -58.633 \\
\hline F50636 & EF080361 & Glossophaga soricina & Guyana & Demerara-Mahaic & c Ceiba Biological Ce 6.5 & -58.217 \\
\hline F50676 & JF435398 & Glossophaga soricina & Guyana & Demerara-Mahaic & c Ceiba Biological Ce 6.5 & -58.217 \\
\hline F50682 & JF435399 & Glossophaga soricina & Guyana & Demerara-Mahaic & c Ceiba Biological Ce 6.5 & -58.217 \\
\hline
\end{tabular}




\begin{tabular}{|c|c|c|c|c|c|c|}
\hline F50694 & JF435400 & Glossophaga soricina & Guyana & \multicolumn{2}{|c|}{ Demerara-Mahaic Ceiba Biological Ce 6.5} & -58.217 \\
\hline F50732 & JF435401 & Glossophaga soricina & Guyana & \multicolumn{2}{|c|}{ Demerara-Mahaic Ceiba Biological Ce 6.5} & -58.217 \\
\hline F50735 & JF435402 & Glossophaga soricina & Guyana & \multicolumn{2}{|c|}{ Demerara-Mahaic Ceiba Biological Ce 6.5} & -58.217 \\
\hline F50748 & JF435403 & Glossophaga soricina & Guyana & \multicolumn{2}{|c|}{ Demerara-Mahaic Ceiba Biological Ce 6.5} & -58.217 \\
\hline F50757 & JF435404 & Glossophaga soricina & Guyana & \multicolumn{2}{|c|}{ Demerara-Mahaic Ceiba Biological Ce 6.5} & -58.217 \\
\hline F50796 & JF435405 & Glossophaga soricina & Guyana & \multicolumn{2}{|c|}{ Demerara-Mahaic Ceiba Biological Ce 6.5} & -58.217 \\
\hline F50801 & JF435406 & Glossophaga soricina & Guyana & \multicolumn{2}{|c|}{ Demerara-Mahaic Ceiba Biological Ce 6.5} & -58.217 \\
\hline F50806 & JF435407 & Glossophaga soricina & Guyana & \multicolumn{2}{|c|}{ Demerara-Mahaic Ceiba Biological Ce 6.5} & -58.217 \\
\hline F50807 & JF435408 & Glossophaga soricina & Guyana & \multicolumn{2}{|c|}{ Demerara-Mahaic Ceiba Biological Ce 6.5} & -58.217 \\
\hline F50808 & JF435409 & Glossophaga soricina & Guyana & Demerara-Mahai & Ceiba Biological Ce 6.5 & -58.217 \\
\hline F50809 & JF435410 & Glossophaga soricina & Guyana & Demerara-Mahai & Ceiba Biological Ce 6.5 & -58.217 \\
\hline F51168 & JF435391 & Glossophaga soricina & Guyana & Potaro-Siparuni & Kaieteur National P: 5.175 & -59.481 \\
\hline F51216 & JF435392 & Glossophaga soricina & Guyana & Potaro-Siparuni & Kaieteur National P: 5.175 & -59.481 \\
\hline F51221 & JF435393 & Glossophaga soricina & Guyana & Potaro-Siparuni & Kaieteur National P: 5.175 & -59.481 \\
\hline F51228 & JF435394 & Glossophaga soricina & Guyana & Potaro-Siparuni & Kaieteur National P: 5.175 & -59.481 \\
\hline F51233 & JF435395 & Glossophaga soricina & Guyana & Potaro-Siparuni & Kaieteur National P: 5.175 & -59.481 \\
\hline F51234 & JF435396 & Glossophaga soricina & Guyana & Potaro-Siparuni & Kaieteur National P: 5.175 & -59.481 \\
\hline F51235 & JF435397 & Glossophaga soricina & Guyana & Potaro-Siparuni & Kaieteur National P: 5.175 & -59.481 \\
\hline F51252 & JF435388 & Glossophaga soricina & Guyana & Potaro-Siparuni & Kaieteur National Pi 5.175 & -59.481 \\
\hline F51304 & JF435389 & Glossophaga soricina & Guyana & Potaro-Siparuni & Kaieteur National P: 5.175 & -59.481 \\
\hline F51316 & JF435390 & Glossophaga soricina & Guyana & Potaro-Siparuni & Kaieteur National P: 5.175 & -59.481 \\
\hline F51480 & JF454694 & Glossophaga soricina & Guyana & Upper Takutu-Up & Dadanawa Ranch 2.824 & -59.526 \\
\hline F51481 & JF454678 & Glossophaga soricina & Guyana & Upper Takutu-Up & Dadanawa Ranch & -59.526 \\
\hline F51493 & JF454690 & Glossophaga soricina & Guyana & Upper Takutu-Up & Dadanawa Ranch & -59.526 \\
\hline F51494 & JF454689 & Glossophaga soricina & Guyana & Upper Takutu-Up & Dadanawa Ranch & -59.526 \\
\hline F51495 & JF454688 & Glossophaga soricina & Guyana & Upper Takutu-Up & Dadanawa Ranch & -59.526 \\
\hline F51496 & JF454686 & Glossophaga soricina & Guyana & Upper Takutu-Up & Dadanawa Ranch & -59.526 \\
\hline F51497 & JF454683 & Glossophaga soricina & Guyana & Upper Takutu-Up & Dadanawa Ranch & -59.526 \\
\hline F51498 & JF454680 & Glossophaga soricina & Guyana & Upper Takutu-Up & Dadanawa Ranch & -59.526 \\
\hline F51537 & JF454704 & Glossophaga soricina & Guyana & Upper Takutu-Up & Dadanawa Ranch & -59.526 \\
\hline F51538 & JF454703 & Glossophaga soricina & Guyana & Upper Takutu-Up & Dadanawa Ranch & -59.526 \\
\hline F51539 & JF454702 & Glossophaga soricina & Guyana & Upper Takutu-Up & Dadanawa Ranch & -59.526 \\
\hline F51556 & JF454695 & Glossophaga soricina & Guyana & Upper Takutu-Up & Dadanawa Ranch & -59.526 \\
\hline F51557 & JF454692 & Glossophaga soricina & Guyana & Upper Takutu-Up & Dadanawa Ranch & -59.526 \\
\hline F51558 & JF454691 & Glossophaga soricina & Guyana & Upper Takutu-Up & Dadanawa Ranch & -59.526 \\
\hline F51587 & JF459166 & Glossophaga soricina & Guyana & Upper Takutu-Up & Dadanawa Ranch & -59.526 \\
\hline F51632 & JF454685 & Glossophaga soricina & Guyana & Upper Takutu-Up & $2 \mathrm{Km}$ W Of Unabaitı 2.279 & -59.435 \\
\hline F51678 & JF454701 & Glossophaga soricina & Guyana & Upper Takutu-Up & $2 \mathrm{Km}$ W Of Unabaitı 2.279 & -59.435 \\
\hline F51733 & JF454687 & Glossophaga soricina & Guyana & Upper Takutu-Up & $1 \mathrm{Km}$ Upstream Fro 2.486 & -59.476 \\
\hline F51752 & JF454698 & Glossophaga soricina & Guyana & Upper Takutu-Up & $1 \mathrm{Km}$ Upstream Fro 2.486 & -59.476 \\
\hline F51753 & JF454681 & Glossophaga soricina & Guyana & Upper Takutu-Up & $1 \mathrm{Km}$ Upstream Fro 2.486 & -59.476 \\
\hline F51754 & JF454700 & Glossophaga soricina & Guyana & Upper Takutu-Up & $1 \mathrm{Km}$ Upstream Fro 2.486 & -59.476 \\
\hline F51774 & JF454677 & Glossophaga soricina & Guyana & Upper Takutu-Up & $1 \mathrm{Km}$ Upstream Fro 2.486 & -59.476 \\
\hline F51802 & JF454684 & Glossophaga soricina & Guyana & Upper Takutu-Up & $1 \mathrm{Km}$ Upstream Fro 2.486 & -59.476 \\
\hline F51803 & JF454682 & Glossophaga soricina & Guyana & Upper Takutu-Up & $1 \mathrm{Km}$ Upstream Fro 2.486 & -59.476 \\
\hline F51804 & JF454679 & Glossophaga soricina & Guyana & Upper Takutu-Up & $1 \mathrm{Km}$ Upstream Fro 2.486 & -59.476 \\
\hline F51827 & JF454697 & Glossophaga soricina & Guyana & Upper Takutu-Up & $1 \mathrm{Km}$ Upstream Fro 2.486 & -59.476 \\
\hline F51828 & JF454696 & Glossophaga soricina & Guyana & Upper Takutu-Up & $1 \mathrm{Km}$ Upstream Fro 2.486 & -59.476 \\
\hline F51829 & JF454693 & Glossophaga soricina & Guyana & Upper Takutu-Up & $1 \mathrm{Km}$ Upstream Fro 2.486 & -59.476 \\
\hline F51848 & & Glossophaga soricina & Guyana & Upper Takutu-Up & $1 \mathrm{Km}$ Upstream Fro 2.486 & -59.476 \\
\hline F51850 & JF454699 & Glossophaga soricina & Guyana & Upper Takutu-Up & $1 \mathrm{Km}$ Upstream Fro 2.486 & -59.476 \\
\hline F52661 & JF459165 & Glossophaga soricina & Guyana & Potaro-Siparuni & Iwokrama Forest, C 4.25 & -58.909 \\
\hline F52675 & JF459164 & Glossophaga soricina & Guyana & Potaro-Siparuni & Iwokrama Forest, C 4.25 & -58.909 \\
\hline F52718 & JF459163 & Glossophaga soricina & Guyana & Potaro-Siparuni & Iwokrama Forest, C 4.25 & -58.909 \\
\hline FN31131 & JF435451 & Glossophaga soricina & Guyana & Upper Demerara & I Kurupukari, East Ba 4.667 & -58.683 \\
\hline FN31132 & JF435452 & Glossophaga soricina & Guyana & Upper Demerara & I Kurupukari, East Ba 4.667 & -58.683 \\
\hline FN31156 & JF435453 & Glossophaga soricina & Guyana & Upper Demerara & I Kurupukari, East $\mathrm{Ba} 4.667$ & -58.683 \\
\hline FN31157 & JF435454 & Glossophaga soricina & Guyana & Upper Demerara & I Kurupukari, East $\mathrm{Ba} 4.667$ & -58.683 \\
\hline FN31520 & JF435455 & Glossophaga soricina & Guyana & Barima-Waini & Kumaka $\quad 7.65$ & -58.95 \\
\hline FN31580 & JF435456 & Glossophaga soricina & Guyana & Barima-Waini & Kwabanna $\quad 7.567$ & -59.15 \\
\hline FN33323 & JF435445 & Glossophaga soricina & Guyana & Upper Takutu-Up & Kuma River, 5 Mi E 3.267 & -59.717 \\
\hline FN33340 & JF435446 & Glossophaga soricina & Guyana & Upper Takutu-Up & Kuma River, 5 Mi E 3.267 & -59.717 \\
\hline FN33341 & JF435447 & Glossophaga soricina & Guyana & Upper Takutu-Up & Kuma River, 5 Mi E 3.267 & -59.717 \\
\hline FN33368 & JF435448 & Glossophaga soricina & Guyana & Upper Takutu-Up & Kuma River, 5 Mi E 3.267 & -59.717 \\
\hline FN33418 & JF435449 & Glossophaga soricina & Guyana & Upper Takutu-Up & Karanambo $\quad 3.75$ & -59.3 \\
\hline FN33419 & JF435450 & Glossophaga soricina & Guyana & Upper Takutu-Up & Karanambo & -59.3 \\
\hline F53477 & KT023195 & Glossophaga soricina & JAMAICA & SAINT CATHER & ST. CLAIR CAVE, E 18.15056 & -77.0743 \\
\hline F53478 & KT023196 & Glossophaga soricina & JAMAICA & SAINT CATHER & ST. CLAIR CAVE, E 18.15056 & -77.0743 \\
\hline F53488 & КT023197 & Glossophaga soricina & JAMAICA & SAINT ELIZABE & 14 KM NW OF BAL 1823441 & -777643 \\
\hline F53496 & КT023198 & Glossonhaga soricina & JAMAICA & SAINT ELIZABE & $14 \mathrm{KM} N W \mathrm{OF}$ BAL 1823441 & -777643 \\
\hline & & & & & & \\
\hline F53505 & KT023199 & Glossophaga soricina & JAMAICA & WESTMORELAI & I 13 KM NW OF BLU 18.28515 & -78.0549 \\
\hline F53506 & KT023200 & Glossophaga soricina & JAMAICA & WESTMORELAI & | 13 KM NW OF BLU 18.28515 & -78.0549 \\
\hline F53513 & KT023201 & Glossophaga soricina & JAMAICA & SAINT ANN & 1 KM N OF QUEEN 18.44363 & -77.4068 \\
\hline
\end{tabular}




\begin{tabular}{|c|c|c|c|c|c|c|}
\hline F53515 & KT023202 & Glossophaga soricina & JAMAICA & SAINT ANN & 4 KM NE OF QUEE 18.46348 & -77.3892 \\
\hline F53517 & KT023203 & Glossophaga soricina & JAMAICA & PORTLAND & SHERWOOD FORE 18.14076 & -76.3761 \\
\hline F53523 & KT023204 & Glossophaga soricina & JAMAICA & PORTLAND & SHERWOOD FORE 18.14076 & -76.3761 \\
\hline FN29997 & JF435387 & Glossophaga soricina & Mexico & Quintana Roo & $1 \mathrm{Km} \mathrm{S}, 10 \mathrm{Km} \mathrm{WC} 20.85$ & -89 \\
\hline FN30015 & JF435473 & Glossophaga soricina & Mexico & Campeche & $5.5 \mathrm{Km} \mathrm{N}, 0.5 \mathrm{Km} \mathrm{E} 18.15$ & -92.1 \\
\hline FN30016 & JF435474 & Glossophaga soricina & Mexico & Campeche & $5.5 \mathrm{Km} \mathrm{N}, 0.5 \mathrm{Km} \mathrm{E} 18.15$ & -92.1 \\
\hline FN30017 & JF435475 & Glossophaga soricina & Mexico & Campeche & $5.5 \mathrm{Km} \mathrm{N}, 0.5 \mathrm{Km} \mathrm{E} 18.15$ & -92.1 \\
\hline FN30018 & JF435472 & Glossophaga soricina & Mexico & Campeche & $5.5 \mathrm{Km} \mathrm{N}, 0.5 \mathrm{Km} \mathrm{E} 18.15$ & -92.1 \\
\hline FN30019 & JF435376 & Glossophaga soricina & Mexico & Campeche & Jonuta, Tabasco, 5. 18.15 & -92.1 \\
\hline FN30020 & JF435377 & Glossophaga soricina & Mexico & Campeche & $5.5 \mathrm{Km} \mathrm{N}, 0.5 \mathrm{Km} \mathrm{E} 18.15$ & -92.1 \\
\hline FN30021 & JF435378 & Glossophaga soricina & Mexico & Campeche & $5.5 \mathrm{Km} \mathrm{N}, 0.5 \mathrm{Km} \mathrm{E} 18.15$ & -92.1 \\
\hline FN30022 & JF435379 & Glossophaga soricina & Mexico & Campeche & $5.5 \mathrm{Km} \mathrm{N}, 0.5 \mathrm{Km} \mathrm{E} 18.15$ & -92.1 \\
\hline FN30023 & JF435380 & Glossophaga soricina & Mexico & Campeche & $5.5 \mathrm{Km} \mathrm{N}, 0.5 \mathrm{Km} \mathrm{E} 18.15$ & -92.1 \\
\hline FN30024 & JF435381 & Glossophaga soricina & Mexico & Campeche & $5.5 \mathrm{Km} \mathrm{N}, 0.5 \mathrm{Km} \mathrm{E} 18.15$ & -92.1 \\
\hline FN30025 & JF435382 & Glossophaga soricina & Mexico & Campeche & $5.5 \mathrm{Km} \mathrm{N}, 0.5 \mathrm{Km} \mathrm{E} 18.15$ & -92.1 \\
\hline FN30890 & JF435383 & Glossophaga soricina & Mexico & Quintana Roo & $6 \mathrm{Km} \mathrm{S}$ Of Majahua 18.683 & -87.733 \\
\hline FN30921 & JF435384 & Glossophaga soricina & Mexico & Quintana Roo & Laguna Noh-Bec, 219.133 & -88.183 \\
\hline FN30922 & JF435385 & Glossophaga soricina & Mexico & Quintana Roo & Laguna Noh-Bec, 219.133 & -88.183 \\
\hline FN30923 & JF435485 & Glossophaga soricina & Mexico & Quintana Roo & Laguna Noh-Bec, 219.133 & -88.183 \\
\hline FN33162 & JF435386 & Glossophaga soricina & Mexico & Chiapas & Puerto Arista $\quad 15.917$ & -93.8 \\
\hline F48056 & JF448040 & Glossophaga soricina & Nicaragua & Rivas & & \\
\hline F48057 & JF448041 & Glossophaga soricina & Nicaragua & Rivas & & \\
\hline F38055 & JF435358 & Glossophaga soricina & Panama & Canal Zone & Gamboa & -79.7 \\
\hline F38056 & JF435467 & Glossophaga soricina & Panama & Canal Zone & Gamboa & -79.7 \\
\hline F40961 & JF435477 & Glossophaga soricina & Suriname & Brokopondo & Brownsberg Nature 4.95 & -55.183 \\
\hline F40962 & JF435478 & Glossophaga soricina & Suriname & Brokopondo & Brownsberg Nature 4.95 & -55.183 \\
\hline F40973 & JF448045 & Glossophaga soricina & Suriname & Brokopondo & Brownsberg Nature 4.95 & -55.183 \\
\hline F41299 & JF435479 & Glossophaga soricina & Suriname & Brokopondo & Brownsberg Nature 4.917 & -55.167 \\
\hline F52431 & $J Q 601353$ & Glossophaga soricina & Suriname & Sipaliwini & Tafelberg, Augustus 3.93 & -56.19 \\
\hline F52443 & JQ601361 & Glossophaga soricina & Suriname & Sipaliwini & Tafelberg, Augustus 3.93 & -56.19 \\
\hline F52454 & JQ601366 & Glossophaga soricina & Suriname & Sipaliwini & Tafelberg, Augustus 3.93 & -56.19 \\
\hline F52458 & JQ601369 & Glossophaga soricina & Suriname & Sipaliwini & Tafelberg, Augustus 3.93 & -56.19 \\
\hline F52502 & JQ601377 & Glossophaga soricina & Suriname & Sipaliwini & Tafelberg, Augustus 3.93 & -56.19 \\
\hline F52514 & \multicolumn{2}{|c|}{ ABSRA664-0 Glossophaga soricina } & Suriname & Sipaliwini & Tafelberg, Augustus 3.93 & -56.19 \\
\hline F52582 & & Glossophaga soricina & Suriname & Sipaliwini & Tafelberg, Caiman ( 3.899 & -56.181 \\
\hline F52604 & JQ601425 & Glossophaga soricina & Suriname & Sipaliwini & Tafelberg, Caiman ( 3.899 & -56.181 \\
\hline F52870 & HQ545556 & Glossophaga soricina & Suriname & Sipaliwini & Kushere Landing, S 1.944 & -56.063 \\
\hline F52890 & HQ545567 & Glossophaga soricina & Suriname & Sipaliwini & Kushere Landing, S 1.944 & -56.063 \\
\hline F52913 & HQ545588 & Glossophaga soricina & Suriname & Sipaliwini & Kushere Landing, S 1.944 & -56.063 \\
\hline F52914 & HQ545589 & Glossophaga soricina & Suriname & Sipaliwini & Kushere Landing, S 1.944 & -56.063 \\
\hline F52926 & HQ545601 & Glossophaga soricina & Suriname & Sipaliwini & Kushere Landing, S 1.944 & -56.063 \\
\hline F52927 & HQ545602 & Glossophaga soricina & Suriname & Sipaliwini & Kushere Landing, S 1.944 & -56.063 \\
\hline F52940 & HQ545615 & Glossophaga soricina & Suriname & Sipaliwini & Kushere Landing, S 1.944 & -56.063 \\
\hline F52941 & HQ545616 & Glossophaga soricina & Suriname & Sipaliwini & Kushere Landing, S 1.944 & -56.063 \\
\hline F52944 & HQ545618 & Glossophaga soricina & Suriname & Sipaliwini & Kushere Landing, S 1.944 & -56.063 \\
\hline F52945 & HQ545619 & Glossophaga soricina & Suriname & Sipaliwini & Kushere Landing, S 1.944 & -56.063 \\
\hline F52959 & HQ545632 & Glossophaga soricina & Suriname & Sipaliwini & Kushere Landing, S 1.944 & -56.063 \\
\hline F52964 & HQ545635 & Glossophaga soricina & Suriname & Sipaliwini & Kushere Landing, S 1.944 & -56.063 \\
\hline F52980 & HQ545648 & Glossophaga soricina & Suriname & Sipaliwini & Kushere Landing, S 1.944 & -56.063 \\
\hline F52996 & HQ545663 & Glossophaga soricina & Suriname & Sipaliwini & Kushere Landing, S 1.944 & -56.063 \\
\hline F53017 & HQ545682 & Glossophaga soricina & Suriname & Sipaliwini & Kushere Landing, S 1.944 & -56.063 \\
\hline F53030 & HQ545448 & Glossophaga soricina & Suriname & Sipaliwini & Kushere Landing, S 1.944 & -56.063 \\
\hline F53056 & HQ545470 & Glossophaga soricina & Suriname & Sipaliwini & Iconja Landing, Sipc 1.994 & -56.092 \\
\hline F53096 & HQ919669 & Glossophaga soricina & Suriname & Sipaliwini & Iconja Landing, Sipc 1.994 & -56.092 \\
\hline F53098 & HQ919671 & Glossophaga soricina & Suriname & Sipaliwini & Iconja Landing, Sipc 1.994 & -56.092 \\
\hline F53134 & HQ919704 & Glossophaga soricina & Suriname & Sipaliwini & Iconja Landing, Sipc 1.994 & -56.092 \\
\hline F53195 & HQ919757 & Glossophaga soricina & Suriname & Sipaliwini & $\begin{array}{ll}\text { Sipaliwini Village } & 2.026\end{array}$ & -56.124 \\
\hline F54389 & JF435357 & Glossophaga soricina & Suriname & Nickerie & Bakhuis, Transect 94.478 & -57.042 \\
\hline F54449 & JF435356 & Glossophaga soricina & Suriname & Nickerie & Bakhuis, Transect 74.529 & -56.904 \\
\hline F54498 & EU096746 & Glossophaga soricina & Suriname & Sipaliwini & Bakhuis, Transect 14.661 & -57.178 \\
\hline F54517 & EU096750 & Glossophaga soricina & Suriname & Sipaliwini & Bakhuis, Transect 14.661 & -57.178 \\
\hline F54893 & EU096748 & Glossophaga soricina & Suriname & Sipaliwini & Blanche Marie Falls 4.811 & -56.994 \\
\hline F54945 & EU096744 & Glossophaga soricina & Suriname & Sipaliwini & Blanche Marie Falls 4.811 & -56.994 \\
\hline F54950 & EU096749 & Glossophaga soricina & Suriname & Sipaliwini & Blanche Marie Falls 4.811 & -56.994 \\
\hline F54984 & EU096747 & Glossophaga soricina & Suriname & Sipaliwini & Blanche Marie Falls 4.811 & -56.994 \\
\hline F54985 & EU096743 & Glossophaga soricina & Suriname & Sipaliwini & Blanche Marie Falls 4.811 & -56.994 \\
\hline F54986 & EU096745 & Glossophaga soricina & Suriname & Sipaliwini & Blanche Marie Falls 4.811 & -56.994 \\
\hline F42935 & JF435480 & Glossophaga soricina & Venezuela & Amazonas & Pozon, 50 Km Ne $\mathrm{C} 6.05$ & -67.417 \\
\hline F43051 & JF435482 & Glossophaga soricina & Venezuela & Bolivar & Hato La Florida, 357.567 & -65.867 \\
\hline F43132 & JF435481 & Glossophaga soricina & Venezuela & Guarico & Rio Orituco, $10 \mathrm{Km} 9.4$ & -66.467 \\
\hline F58968 & KX355043 & Lasiurus borealis & Dominican Republic & Pedernales & $1 \mathrm{~km}$ SE of Aguas $\wedge 18.16282$ & -71.6905 \\
\hline F58969 & KX355044 & Lasiurus borealis & Dominican Republic & Pedernales & $1 \mathrm{~km}$ SE of Aguas $\wedge 18.16282$ & -71.6905 \\
\hline F58970 & KX355045 & Lasiurus borealis & Dominican Republic & Pedernales & $1 \mathrm{~km} \mathrm{SE}$ of Aguas $\wedge 18.16282$ & -71.6905 \\
\hline F59203 & KX355046 & Lasiurus borealis & Dominican Republic & La Vega & Parque Nacional Ar 19.06727 & -70.8632 \\
\hline F58960 & KX355047 & Macrotus waterhousii & Dominican Republic & Pedernales & $9 \mathrm{~km} \mathrm{NE}$ of Pederna 18.08317 & -71.6709 \\
\hline F58975 & KX355048 & Macrotus waterhousii & Dominican Republic & Pedernales & Cueva de Marazate 17.96624 & -71.6355 \\
\hline F58976 & & Macrotus waterhousii & & Pedernales & & \\
\hline
\end{tabular}




\begin{tabular}{|c|c|c|c|c|c|c|}
\hline F58983 & KX355050 & Macrotus waterhousii & Dominican Republic & Barahona & Cueva de los Patos 17.96002 & -71.1833 \\
\hline F58984 & KX355051 & Macrotus waterhousii & Dominican Republic & Barahona & Cueva de los Patos 17.96002 & -71.1833 \\
\hline F58985 & KX355052 & Macrotus waterhousii & Dominican Republic & Barahona & Cueva de los Patos 17.96002 & -71.1833 \\
\hline F59237 & KX355053 & Macrotus waterhousii & Dominican Republic & Monte Plata & Cueva la Chepa, 1418.86914 & -69.5763 \\
\hline F59238 & KX355054 & Macrotus waterhousii & Dominican Republic & Monte Plata & Cueva la Chepa, 1418.86914 & -69.5763 \\
\hline F59239 & KX355055 & Macrotus waterhousii & Dominican Republic & Monte Plata & Cueva la Chepa, 1418.86914 & -69.5763 \\
\hline F59278 & KX355056 & Macrotus waterhousii & Dominican Republic & La Altagracia & Cueva Taina 3km S 18.76605 & -68.5482 \\
\hline F59279 & KX355057 & Macrotus waterhousii & Dominican Republic & La Altagracia & Cueva Taina 3km S 18.76605 & -68.5482 \\
\hline F59280 & KX355058 & Macrotus waterhousii & Dominican Republic & La Altagracia & Cueva Taina 3km S 18.76605 & -68.5482 \\
\hline & JF442236 & Molossus & Ecuador & & & \\
\hline & JF442237 & Molossus & Ecuador & & & \\
\hline & JF442238 & Molossus & Ecuador & & & \\
\hline & JF442239 & Molossus & Ecuador & & & \\
\hline & JF442240 & Molossus & Ecuador & & & \\
\hline & JF442241 & Molossus & Ecuador & & & \\
\hline & JF442242 & Molossus & Ecuador & & & \\
\hline & JF442243 & Molossus & Ecuador & & & \\
\hline & JF442244 & Molossus & Ecuador & & & \\
\hline & JF442245 & Molossus & Ecuador & & & \\
\hline F37630 & JF448947 & Molossus coibensis & Ecuador & Napo & Parque Nacional Ya -0.833 & -76.35 \\
\hline F37882 & JF448950 & Molossus coibensis & Ecuador & Napo & Parque Nacional Ya -0.65 & -76.45 \\
\hline F37883 & JF448088 & Molossus coibensis & Ecuador & Napo & Parque Nacional Ya - 0.65 & -76.45 \\
\hline F37933 & JF448948 & Molossus coibensis & Ecuador & Napo & Parque Nacional Ya -0.683 & -76.4 \\
\hline F37934 & JF448946 & Molossus coibensis & Ecuador & Napo & Parque Nacional Ya -0.683 & -76.4 \\
\hline F37936 & JF448949 & Molossus coibensis & Ecuador & Napo & Parque Nacional Ya -0.683 & -76.4 \\
\hline F41830 & JF448951 & Molossus coibensis & Ecuador & Orellana & $42 \mathrm{Km} \mathrm{S}, 9.5 \mathrm{Km} \mathrm{E}-0.68$ & -76.382 \\
\hline F41831 & \multicolumn{2}{|c|}{ ABRMM099-ו Molossus coibensis } & Ecuador & Orellana & $42 \mathrm{Km} \mathrm{S}, 9.5 \mathrm{Km} \mathrm{E}-0.68$ & -76.382 \\
\hline F41832 & \multicolumn{2}{|r|}{ ABRMM100-ו Molossus coibensis } & Ecuador & Orellana & $42 \mathrm{Km} \mathrm{S}, 9.5 \mathrm{Km} \mathrm{E}-0.68$ & -76.382 \\
\hline V-1919 & \multicolumn{2}{|r|}{ ABFG423-10 Molossus coibensis } & French Guiana & Cayenne & Kaw $\quad 4.56528$ & -52.1894 \\
\hline V-1997 & \multicolumn{2}{|r|}{ ABFG434-10 Molossus coibensis } & French Guiana & Saint-Laurent-du- & I Angouleme & -53.65 \\
\hline V-2550 & \multicolumn{2}{|r|}{ ABFG554-10 Molossus coibensis } & French Guiana & Cayenne & \multicolumn{2}{|l|}{ pK-14 route des $\mathrm{Pla}$} \\
\hline F35095 & & BCBN360-05 Molossus coibensis & Guyana & Barima-Waini & Baramita, Old Worlc 7.367 & -60.483 \\
\hline F51451 & & ABGYG584-C Molossus coibensis & Guyana & Upper Takutu-Upr & Dadanawa Ranch 2.824 & -59.526 \\
\hline F58948 & KX355059 & Molossus molossus & Dominican Republic & National District & Santo Domingo, Jar 18.49399 & -69.9561 \\
\hline F58949 & KX355060 & Molossus molossus & Dominican Republic & National District & Santo Domingo, Jar 18.49399 & -69.9561 \\
\hline F58950 & KX355061 & Molossus molossus & Dominican Republic & National District & Santo Domingo, Jar 18.49399 & -69.9561 \\
\hline F58951 & KX355062 & Molossus molossus & Dominican Republic & National District & Santo Domingo, Jar 18.49399 & -69.9561 \\
\hline F59247 & KX355063 & Molossus molossus & Dominican Republic & El Seibo & $7 \mathrm{~km}$ SE of Miches $\quad 18.9254$ & -69.0078 \\
\hline F59248 & KX355064 & Molossus molossus & Dominican Republic & El Seibo & $7 \mathrm{~km}$ SE of Miches & -69.0078 \\
\hline F59249 & KX355065 & Molossus molossus & Dominican Republic & El Seibo & $7 \mathrm{~km}$ SE of Miches & -69.0078 \\
\hline F59250 & KX355066 & Molossus molossus & Dominican Republic & El Seibo & $7 \mathrm{~km}$ SE of Miches & -69.0078 \\
\hline & JF442198 & Molossus molossus & Ecuador & & & \\
\hline & JF442199 & Molossus molossus & Ecuador & & & \\
\hline & JF442200 & Molossus molossus & Ecuador & & & \\
\hline & JF442201 & Molossus molossus & Ecuador & & & \\
\hline & JF442202 & Molossus molossus & Ecuador & & & \\
\hline & JF442203 & Molossus molossus & Ecuador & & & \\
\hline & JF442204 & Molossus molossus & Ecuador & & & \\
\hline & JF442205 & Molossus molossus & Ecuador & & & \\
\hline & JF442206 & Molossus molossus & Ecuador & & & \\
\hline & JF442207 & Molossus molossus & Ecuador & & & \\
\hline & JF442208 & Molossus molossus & Ecuador & & & \\
\hline & JF442209 & Molossus molossus & Ecuador & & & \\
\hline & JF442210 & Molossus molossus & Ecuador & & & \\
\hline & $\mathrm{JF} 442211$ & Molossus molossus & Ecuador & & & \\
\hline & JF442212 & Molossus molossus & Ecuador & & & \\
\hline & JF442213 & Molossus molossus & Ecuador & & & \\
\hline & JF442214 & Molossus molossus & Ecuador & & & \\
\hline & JF442215 & Molossus molossus & Ecuador & & & \\
\hline & JF442216 & Molossus molossus & Ecuador & & & \\
\hline & JF442217 & Molossus molossus & Ecuador & & & \\
\hline & JF442218 & Molossus molossus & Ecuador & & & \\
\hline & JF442220 & Molossus molossus & Ecuador & & & \\
\hline & JF442221 & Molossus molossus & Ecuador & & & \\
\hline & JF442222 & Molossus molossus & Ecuador & & & \\
\hline & JF442224 & Molossus molossus & Ecuador & & & \\
\hline & JF442227 & Molossus molossus & Ecuador & & & \\
\hline & JF442228 & Molossus molossus & Ecuador & & & \\
\hline & JF442229 & Molossus molossus & Ecuador & & & \\
\hline & JF442230 & Molossus molossus & Ecuador & & & \\
\hline & JF442231 & Molossus molossus & Ecuador & & & \\
\hline & JF442232 & Molossus molossus & Ecuador & & & \\
\hline & JF442233 & Molossus molossus & Ecuador & & & \\
\hline & JF442234 & Molossus molossus & Ecuador & & & \\
\hline & JF442235 & Molossus molossus & Ecuador & & & \\
\hline F37112 & JF448961 & Molossus molossus & Ecuador & Napo & Parque Nacional Ya - 0.65 & -76.45 \\
\hline F37117 & JF448963 & Molossus molossus & Ecuador & Napo & Parque Nacional Ya -0.65 & -76.45 \\
\hline F37273 & JF448960 & Molossus molossus & Ecuador & Napo & Parque Nacional Ya -0.667 & -76.467 \\
\hline F37685 & JF448968 & Molossus molossus & Ecuador & Napo & Parque Nacional Ya -0.65 & -76.45 \\
\hline F37689 & JF448964 & Molossus molossus & Ecuador & Napo & Parque Nacional Ya-0.65 & -76.45 \\
\hline
\end{tabular}




\begin{tabular}{|c|c|c|c|c|c|c|}
\hline F37696 & JF448962 & Molossus molossus & Ecuador & Napo & Parque Nacional Ya -0.65 & -76.45 \\
\hline F37747 & JF448965 & Molossus molossus & Ecuador & Napo & Parque Nacional Ya - 0.65 & -76.45 \\
\hline F37776 & JF448955 & Molossus molossus & Ecuador & Napo & Parque Nacional Ya -0.833 & -76.35 \\
\hline F37777 & JF448957 & Molossus molossus & Ecuador & Napo & Parque Nacional Ya - 0.833 & -76.35 \\
\hline F37778 & JF448958 & Molossus molossus & Ecuador & Napo & Parque Nacional Ya - 0.833 & -76.35 \\
\hline F37779 & JF448959 & Molossus molossus & Ecuador & Napo & Parque Nacional Ya - 0.833 & -76.35 \\
\hline F37810 & JF448954 & Molossus molossus & Ecuador & Napo & Parque Nacional Ya -0.65 & -76.45 \\
\hline F37811 & JF448956 & Molossus molossus & Ecuador & Napo & Parque Nacional Ya - 0.65 & -76.45 \\
\hline F37833 & JF448952 & Molossus molossus & Ecuador & Napo & Parque Nacional Ya -0.833 & -76.35 \\
\hline F37935 & JF448953 & Molossus molossus & Ecuador & Napo & Parque Nacional Ya-0.683 & -76.4 \\
\hline F40129 & JF448966 & Molossus molossus & Ecuador & Napo & Parque Nacional Ya - 0.65 & -76.45 \\
\hline F40177 & JF448967 & Molossus molossus & Ecuador & Napo & Parque Nacional Ya - 0.65 & -76.45 \\
\hline F40307 & JF448970 & Molossus molossus & Ecuador & Napo & Parque Nacional Ya -0.65 & -76.45 \\
\hline F40308 & JF448969 & Molossus molossus & Ecuador & Napo & Parque Nacional Ya -0.65 & -76.45 \\
\hline F40478 & JF448974 & Molossus molossus & Ecuador & Napo & Parque Nacional Ya & \\
\hline F40479 & JF448971 & Molossus molossus & Ecuador & Napo & Parque Nacional Ya & \\
\hline F40480 & JF448973 & Molossus molossus & Ecuador & Napo & Parque Nacional Ya & \\
\hline F41836 & JF448977 & Molossus molossus & Ecuador & Orellana & $42 \mathrm{Km} \mathrm{S}, 9.5 \mathrm{Km} \mathrm{E}-0.68$ & -76.382 \\
\hline F41837 & JF448976 & Molossus molossus & Ecuador & Orellana & $42 \mathrm{Km} \mathrm{S}, 9.5 \mathrm{Km} \mathrm{E}-0.68$ & -76.382 \\
\hline F41838 & JF448975 & Molossus molossus & Ecuador & Orellana & $32 \mathrm{Km} \mathrm{S}$ Of Pompe! -0.615 & -76.469 \\
\hline F52327 & JF448972 & Molossus molossus & Ecuador & Orellana & $32 \mathrm{Km} \mathrm{S}$ Of Pompe: -0.615 & -76.469 \\
\hline F37019 & JF455081 & Molossus molossus & Guyana & Upper Demerara-I & I Mabura Hill $\quad 5.3$ & -58.7 \\
\hline F37020 & JF455089 & Molossus molossus & Guyana & Upper Demerara-I & -I Mabura Hill & -58.7 \\
\hline F37021 & JF455091 & Molossus molossus & Guyana & Upper Demerara-I & -I Mabura Hill & -58.7 \\
\hline F37022 & JF455070 & Molossus molossus & Guyana & Upper Demerara-I & -I Mabura Hill & -58.7 \\
\hline F37023 & JF455071 & Molossus molossus & Guyana & Upper Demerara-I & -I Mabura Hill & -58.7 \\
\hline F37024 & JF455072 & Molossus molossus & Guyana & Upper Demerara-I & -I Mabura Hill & -58.7 \\
\hline F37025 & JF455073 & Molossus molossus & Guyana & Upper Demerara-I & -I Mabura Hill & -58.7 \\
\hline F37026 & JF455074 & Molossus molossus & Guyana & Upper Demerara-I & -I Mabura Hill & -58.7 \\
\hline F37027 & JF455075 & Molossus molossus & Guyana & Upper Demerara-I & I Mabura Hill & -58.7 \\
\hline F37028 & JF455076 & Molossus molossus & Guyana & Upper Demerara-I & -I Mabura Hill & -58.7 \\
\hline F37029 & JF455077 & Molossus molossus & Guyana & Upper Demerara-I & -I Mabura Hill & -58.7 \\
\hline F37030 & JF455078 & Molossus molossus & Guyana & Upper Demerara-I & -I Mabura Hill & -58.7 \\
\hline F37031 & JF455079 & Molossus molossus & Guyana & Upper Demerara-I & -I Mabura Hill & -58.7 \\
\hline F37032 & JF455080 & Molossus molossus & Guyana & Upper Demerara-I & II Mabura Hill & -58.7 \\
\hline F37033 & JF455082 & Molossus molossus & Guyana & Upper Demerara-I & -I Mabura Hill & -58.7 \\
\hline F37034 & JF455083 & Molossus molossus & Guyana & Upper Demerara-I & -I Mabura Hill & -58.7 \\
\hline F37035 & JF455084 & Molossus molossus & Guyana & Upper Demerara-I & -I Mabura Hill & -58.7 \\
\hline F37036 & JF455085 & Molossus molossus & Guyana & Upper Demerara-I & -I Mabura Hill & -58.7 \\
\hline F37037 & JF455086 & Molossus molossus & Guyana & Upper Demerara-I & I Mabura Hill & -58.7 \\
\hline F37038 & JF455087 & Molossus molossus & Guyana & Upper Demerara-I & - I Mabura Hill & -58.7 \\
\hline F37039 & JF455088 & Molossus molossus & Guyana & Upper Demerara- & -I Mabura Hill & -58.7 \\
\hline F37040 & JF455090 & Molossus molossus & Guyana & Upper Demerara-I & -I Mabura Hill & -58.7 \\
\hline F38551 & JF455049 & Molossus molossus & Guyana & Upper Takutu-Upr & F Gunn'S Strip 1.65 & -58.633 \\
\hline F39270 & & C Molossus molossus & Guyana & Potaro-Siparuni & Iwokrama Reserve, 4.667 & -58.683 \\
\hline F39783 & JF455050 & Molossus molossus & Guyana & Cuyuni-Mazaruni & Kamarang $\quad 5.867$ & -60.617 \\
\hline F39784 & JF455051 & Molossus molossus & Guyana & Cuyuni-Mazaruni & Kamarang & -60.617 \\
\hline F39785 & & C Molossus molossus & Guyana & Cuyuni-Mazaruni & Kamarang & -60.617 \\
\hline F39786 & JF455052 & Molossus molossus & Guyana & Cuyuni-Mazaruni & Kamarang & -60.617 \\
\hline F39787 & JF455023 & Molossus molossus & Guyana & Cuyuni-Mazaruni & Kamarang $\quad 5.867$ & -60.617 \\
\hline F40587 & EF080474 & Molossus molossus & Guyana & Potaro-Siparuni & Field Station, Kurup 4.856 & -58.689 \\
\hline F40588 & EF080475 & Molossus molossus & Guyana & Potaro-Siparuni & Field Station, Kurup 4.856 & -58.689 \\
\hline F40589 & EF080476 & Molossus molossus & Guyana & Potaro-Siparuni & Field Station, Kurup 4.856 & -58.689 \\
\hline F40590 & EF080473 & Molossus molossus & Guyana & Potaro-Siparuni & Field Station, Kurup 4.856 & -58.689 \\
\hline F40591 & EF080477 & Molossus molossus & Guyana & Potaro-Siparuni & Field Station, Kurup 4.856 & -58.689 \\
\hline F40592 & JF455013 & Molossus molossus & Guyana & Potaro-Siparuni & Field Station, Kurup 4.746 & -58.684 \\
\hline F40593 & JF455047 & Molossus molossus & Guyana & Potaro-Siparuni & Field Station, Kurup 4.746 & -58.684 \\
\hline F40594 & JF455036 & Molossus molossus & Guyana & Potaro-Siparuni & Field Station, Kurup 4.746 & -58.684 \\
\hline F40595 & JF455035 & Molossus molossus & Guyana & Potaro-Siparuni & Field Station, Kurup 4.746 & -58.684 \\
\hline F40596 & JF455048 & Molossus molossus & Guyana & Potaro-Siparuni & Field Station, Kurup 4.746 & -58.684 \\
\hline F40597 & JF455046 & Molossus molossus & Guyana & Potaro-Siparuni & Field Station, Kurup 4.746 & -58.684 \\
\hline F40598 & JF455045 & Molossus molossus & Guyana & Potaro-Siparuni & Field Station, Kurup 4.746 & -58.684 \\
\hline F40599 & JF455044 & Molossus molossus & Guyana & Potaro-Siparuni & Field Station, Kurup 4.746 & -58.684 \\
\hline F40600 & JF455043 & Molossus molossus & Guyana & Potaro-Siparuni & Field Station, Kurup 4.746 & -58.684 \\
\hline F40601 & JF455042 & Molossus molossus & Guyana & Potaro-Siparuni & Field Station, Kurup 4.746 & -58.684 \\
\hline F40602 & JF455041 & Molossus molossus & Guyana & Potaro-Siparuni & Field Station, Kurup 4.746 & -58.684 \\
\hline F40603 & JF455040 & Molossus molossus & Guyana & Potaro-Siparuni & Field Station, Kurup 4.746 & -58.684 \\
\hline F40604 & JF455039 & Molossus molossus & Guyana & Potaro-Siparuni & Field Station, Kurup 4.746 & -58.684 \\
\hline F40605 & JF455038 & Molossus molossus & Guyana & Potaro-Siparuni & Field Station, Kurup 4.746 & -58.684 \\
\hline F40606 & JF455037 & Molossus molossus & Guyana & Potaro-Siparuni & Field Station, Kurup 4.746 & -58.684 \\
\hline F45109 & JF455019 & Molossus molossus & Guyana & Potaro-Siparuni & Iwokrama Field Stat 4.667 & -58.683 \\
\hline F45114 & JF455032 & Molossus molossus & Guyana & Potaro-Siparuni & Iwokrama Field Stat 4.667 & -58.683 \\
\hline F45115 & JF455031 & Molossus molossus & Guyana & Potaro-Siparuni & Iwokrama Field Stat 4.667 & -58.683 \\
\hline F45116 & JF455030 & Molossus molossus & Guyana & Potaro-Siparuni & Iwokrama Field Stat 4.667 & -58.683 \\
\hline F45117 & JF455029 & Molossus molossus & Guyana & Potaro-Siparuni & Iwokrama Field Stat 4.667 & -58.683 \\
\hline F45118 & JF455027 & Molossus molossus & Guyana & Potaro-Siparuni & Iwokrama Field Stat 4.667 & -58.683 \\
\hline F45119 & JF455026 & Molossus molossus & Guyana & Potaro-Siparuni & Iwokrama Field Stat 4.667 & -58.683 \\
\hline F45120 & JF455025 & Molossus molossus & Guyana & Potaro-Siparuni & Iwokrama Field Stat 4.667 & -58.683 \\
\hline F45121 & JF455012 & Molossus molossus & Guyana & Potaro-Siparuni & Iwokrama Field Stat 4.667 & -58.683 \\
\hline F45122 & JF455018 & Molossus molossus & Guyana & Potaro-Siparuni & Iwokrama Field Stat 4.667 & -58.683 \\
\hline
\end{tabular}




\begin{tabular}{|c|c|c|c|c|c|c|}
\hline F45164 & JF455034 & Molossus molossus & Guyana & Potaro-Siparuni & Iwokrama Field Stat 4.667 & -58.683 \\
\hline F45248 & EF080471 & Molossus molossus & Guyana & Demerara-Mahaic & c Ceiba Biological Stc 6.5 & -58.217 \\
\hline F45390 & JF455028 & Molossus molossus & Guyana & Demerara-Mahaic & c Georgetown, Botani 6.817 & -58.117 \\
\hline F45391 & JF455033 & Molossus molossus & Guyana & Demerara-Mahaic & c Georgetown, Botani 6.817 & -58.117 \\
\hline F45402 & JF455024 & Molossus molossus & Guyana & Demerara-Mahaic & C Georgetown, Botani 6.817 & -58.117 \\
\hline F50730 & JF455016 & Molossus molossus & Guyana & Demerara-Mahaic & c Ceiba Biological Ce 6.5 & -58.217 \\
\hline F50793 & JF455015 & Molossus molossus & Guyana & Demerara-Mahaic & c Ceiba Biological Ce 6.5 & -58.217 \\
\hline F50794 & JF455017 & Molossus molossus & Guyana & Demerara-Mahaic & c Ceiba Biological Ce 6.5 & -58.217 \\
\hline F51008 & EF080462 & Molossus molossus & Guyana & Potaro-Siparuni & Iwokrama Field Stat 4.683 & -58.683 \\
\hline F51037 & EF080472 & Molossus molossus & Guyana & Potaro-Siparuni & Iwokrama Field Stat 4.683 & -58.683 \\
\hline F51038 & EF080463 & Molossus molossus & Guyana & Potaro-Siparuni & Iwokrama Field Stat 4.683 & -58.683 \\
\hline F51053 & EF080464 & Molossus molossus & Guyana & Potaro-Siparuni & Iwokrama Field Stat 4.683 & -58.683 \\
\hline F51054 & EF080465 & Molossus molossus & Guyana & Potaro-Siparuni & Iwokrama Field Stat 4.683 & -58.683 \\
\hline F51088 & EF080466 & Molossus molossus & Guyana & Potaro-Siparuni & Iwokrama Field Stat 4.683 & -58.683 \\
\hline F51089 & EF080467 & Molossus molossus & Guyana & Potaro-Siparuni & Iwokrama Field Stat 4.683 & -58.683 \\
\hline F51090 & EF080468 & Molossus molossus & Guyana & Potaro-Siparuni & Iwokrama Field Stat 4.683 & -58.683 \\
\hline F51091 & EF080469 & Molossus molossus & Guyana & Potaro-Siparuni & Iwokrama Field Stat 4.683 & -58.683 \\
\hline F51092 & EF080470 & Molossus molossus & Guyana & Potaro-Siparuni & Iwokrama Field Stat 4.683 & -58.683 \\
\hline F51138 & JF455014 & Molossus molossus & Guyana & Potaro-Siparuni & Kaieteur National Pi 5.166 & -59.493 \\
\hline F51247 & JF455020 & Molossus molossus & Guyana & Potaro-Siparuni & Kaieteur National Pi 5.166 & -59.493 \\
\hline F51265 & JF455022 & Molossus molossus & Guyana & Potaro-Siparuni & Kaieteur National P: 5.166 & -59.493 \\
\hline F51567 & $\mathrm{JF} 455021$ & Molossus molossus & Guyana & Upper Takutu-Up & Dadanawa Ranch 2.824 & -59.526 \\
\hline F52686 & JF459213 & Molossus molossus & Guyana & Potaro-Siparuni & Iwokrama Forest, C 4.25 & -58.909 \\
\hline F52687 & JF459212 & Molossus molossus & Guyana & Potaro-Siparuni & Iwokrama Forest, C 4.25 & -58.909 \\
\hline F52689 & JF459211 & Molossus molossus & Guyana & Potaro-Siparuni & Iwokrama Forest, C 4.25 & -58.909 \\
\hline F52690 & JF459210 & Molossus molossus & Guyana & Potaro-Siparuni & Iwokrama Forest, C 4.25 & -58.909 \\
\hline F52691 & JF459209 & Molossus molossus & Guyana & Potaro-Siparuni & Iwokrama Forest, C 4.25 & -58.909 \\
\hline F52692 & JF459208 & Molossus molossus & Guyana & Potaro-Siparuni & Iwokrama Forest, C 4.25 & -58.909 \\
\hline F52724 & JF459207 & Molossus molossus & Guyana & Potaro-Siparuni & Iwokrama Forest, C 4.25 & -58.909 \\
\hline F52725 & JF459206 & Molossus molossus & Guyana & Potaro-Siparuni & Iwokrama Forest, C 4.25 & -58.909 \\
\hline F52760 & JF459205 & Molossus molossus & Guyana & Potaro-Siparuni & Iwokrama Forest, C 4.25 & -58.909 \\
\hline FN31525 & JF455069 & Molossus molossus & Guyana & Barima-Waini & Kwabanna $\quad 7.567$ & -59.15 \\
\hline FN31538 & JF455054 & Molossus molossus & Guyana & Barima-Waini & Kwabanna & -59.15 \\
\hline FN31539 & JF455056 & Molossus molossus & Guyana & Barima-Waini & Kwabanna & -59.15 \\
\hline FN31540 & JF455057 & Molossus molossus & Guyana & Barima-Waini & Kwabanna & -59.15 \\
\hline FN31542 & JF455058 & Molossus molossus & Guyana & Barima-Waini & Kwabanna & -59.15 \\
\hline FN31543 & JF455059 & Molossus molossus & Guyana & Barima-Waini & Kwabanna & -59.15 \\
\hline FN31544 & JF455060 & Molossus molossus & Guyana & Barima-Waini & Kwabanna & -59.15 \\
\hline FN33254 & JF455053 & Molossus molossus & Guyana & Upper Takutu-Up & Lethem, Tabatinga | 3.383 & -59.8 \\
\hline FN33256 & JF455055 & Molossus molossus & Guyana & Upper Takutu-Up & Lethem $\quad 3.383$ & -59.8 \\
\hline FN33283 & JF455061 & Molossus molossus & Guyana & Upper Takutu-Up & Lethem & -59.8 \\
\hline FN33284 & JF455062 & Molossus molossus & Guyana & Upper Takutu-Up & Lethem & -59.8 \\
\hline FN33285 & JF455063 & Molossus molossus & Guyana & Upper Takutu-Up & Lethem & -59.8 \\
\hline FN33286 & JF455064 & Molossus molossus & Guyana & Upper Takutu-Up & Lethem & -59.8 \\
\hline FN33287 & JF455065 & Molossus molossus & Guyana & Upper Takutu-Up & Lethem & -59.8 \\
\hline FN33288 & JF455066 & Molossus molossus & Guyana & Upper Takutu-Up & Lethem & -59.8 \\
\hline FN33289 & JF455067 & Molossus molossus & Guyana & Upper Takutu-Up & Lethem & -59.8 \\
\hline FN33290 & JF455068 & Molossus molossus & Guyana & Upper Takutu-Up & Lethem & -59.8 \\
\hline F53497 & KT023205 & Molossus molossus & JAMAICA & SAINT ELIZABET & $13 \mathrm{KM}$ NW OF BAL، 18.22700 & -77.7545 \\
\hline F53498 & КT023206 & Molossus molossus & JAMAICA & SAINT ELIZABET & 13 KM NW OF BAL, 18.22700 & -77.7545 \\
\hline F53499 & КT023207 & Molossus molossus & JAMAICA & SAINT ELIZABET & 13 KM NW OF BAL, 18.22700 & -77.7545 \\
\hline F53500 & КT023208 & Molossus molossus & JAMAICA & SAINT ELIZABET & 13 KM NW OF BAL 18.22700 & -77.7545 \\
\hline F53520 & КT023209 & Molossus molossus & JAMAICA & PORTLAND & SHERWOOD FORE 18.14347 & -76.373 \\
\hline F53521 & КT023210 & Molossus molossus & JAMAICA & PORTLAND & SHERWOOD FORE 18.14347 & -76.373 \\
\hline F53522 & КT023211 & Molossus molossus & JAMAICA & PORTLAND & SHERWOOD FORE 18.14347 & -76.373 \\
\hline F53548 & КT023212 & Molossus molossus & JAMAICA & PORTLAND & SHERWOOD FORE 18.14347 & -76.373 \\
\hline F53549 & КT023213 & Molossus molossus & JAMAICA & PORTLAND & SHERWOOD FORE 18.14347 & -76.373 \\
\hline F53550 & КT023214 & Molossus molossus & JAMAICA & PORTLAND & SHERWOOD FORE 18.14347 & -76.373 \\
\hline F53551 & KT023215 & Molossus molossus & JAMAICA & PORTLAND & SHERWOOD FORE 18.14347 & -76.373 \\
\hline $\mathrm{T}-4932$ & HQ918468 ABFG470-10 & Molossus molossus & Martinique & Morne Rouge & Domaine Estripault & \\
\hline V-2091 & HQ918468 & Molossus molossus & Martinique & Morne Rouge & Domaine Estripault & \\
\hline F38021 & JF447423 & Molossus molossus & Panama & Canal Zone & Gamboa $\quad 9.1$ & -79.7 \\
\hline F40974 & JF447683 & Molossus molossus & Suriname & Brokopondo & Brownsberg Nature 4.95 & -55.183 \\
\hline F40975 & JF447681 & Molossus molossus & Suriname & Brokopondo & Brownsberg Nature 4.95 & -55.183 \\
\hline F40976 & JF447684 & Molossus molossus & Suriname & Brokopondo & Brownsberg Nature 4.95 & -55.183 \\
\hline F40977 & JF447680 & Molossus molossus & Suriname & Brokopondo & Brownsberg Nature 4.95 & -55.183 \\
\hline F41025 & $\mathrm{JF} 447682$ & Molossus molossus & Suriname & Brokopondo & Brownsberg Nature 4.95 & -55.183 \\
\hline F52609 & ABGYH117-1 & 1 Molossus molossus & Suriname & Sipaliwini & Tafelberg, Rudi Kap 3.791 & -56.149 \\
\hline F52610 & JQ601428 & Molossus molossus & Suriname & Sipaliwini & Tafelberg, Rudi Kap 3.791 & -56.149 \\
\hline F52611 & JQ601429 & Molossus molossus & Suriname & Sipaliwini & Tafelberg, Rudi Kap 3.791 & -56.149 \\
\hline
\end{tabular}




\begin{tabular}{|c|c|c|c|c|c|c|c|}
\hline F52612 & ABGYH118-1 & 1 Molossus molossus & Suriname & Sipaliwini & Tafelberg, Rudi Kar & 3.791 & -56.149 \\
\hline F53186 & HQ919749 & Molossus molossus & Suriname & Sipaliwini & Sipaliwini Village & 2.026 & -56.124 \\
\hline F53197 & HQ919759 & Molossus molossus & Suriname & Sipaliwini & Sipaliwini Village & 2.026 & -56.124 \\
\hline F53198 & HQ919760 & Molossus molossus & Suriname & Sipaliwini & Sipaliwini Village & 2.026 & -56.124 \\
\hline F53199 & HQ919761 & Molossus molossus & Suriname & Sipaliwini & Sipaliwini Village & 2.026 & -56.124 \\
\hline F53200 & HQ919762 & Molossus molossus & Suriname & Sipaliwini & Sipaliwini Village & 2.026 & -56.124 \\
\hline F53201 & HQ919763 & Molossus molossus & Suriname & Sipaliwini & Sipaliwini Village & 2.026 & -56.124 \\
\hline F53202 & ABSRA1180- & Molossus molossus & Suriname & Sipaliwini & Sipaliwini Village & 2.026 & -56.124 \\
\hline F54977 & EU096793 & Molossus molossus & Suriname & Sipaliwini & Blanche Marie Falls & 4.811 & -56.994 \\
\hline F54978 & EU096791 & Molossus molossus & Suriname & Sipaliwini & Blanche Marie Falls & 4.811 & -56.994 \\
\hline F54979 & EU096794 & Molossus molossus & Suriname & Sipaliwini & Blanche Marie Falls & 4.811 & -56.994 \\
\hline F54980 & EU096789 & Molossus molossus & Suriname & Sipaliwini & Blanche Marie Falls & 4.811 & -56.994 \\
\hline F54981 & EU096792 & Molossus molossus & Suriname & Sipaliwini & Blanche Marie Falls & 4.811 & -56.994 \\
\hline F54982 & EU096790 & Molossus molossus & Suriname & Sipaliwini & Blanche Marie Falls & 4.811 & -56.994 \\
\hline F55003 & EU096788 & Molossus molossus & Suriname & Sipaliwini & Bakhuis Concessior & 4.853 & -56.781 \\
\hline F42969 & JF447834 & Molossus molossus & Venezuela & Amazonas & Pozon, $50 \mathrm{Km} \mathrm{Ne} \mathrm{C}$ & 6.05 & -67.417 \\
\hline F43037 & JF447833 & Molossus molossus & Venezuela & Amazonas & Pozon, $50 \mathrm{Km} \mathrm{Ne} \mathrm{C}$ & 6.05 & -67.417 \\
\hline F43038 & JF447832 & Molossus molossus & Venezuela & Amazonas & Pozon, $50 \mathrm{Km} \mathrm{Ne} \mathrm{C}$ & 6.05 & -67.417 \\
\hline T-4935 & ABFG473-10 & Monophyllus plethodon & Martinique & Gros Morne & Plateau Boucher & & \\
\hline T-4939 & ABFG476-10 & Monophyllus plethodon & Martinique & Fonds Saint Denis & is Piste Forestiere Riv & 14.7172 & -61.0944 \\
\hline T-5126 & HQ918481 ABFG493-10 & Monophyllus plethodon & Martinique & Fonds Saint Denis & ؟Piste ONF Riviere E & 14.7172 & -61.0944 \\
\hline F58977 & KX355067 & Monophyllus redmani & Dominican Republic & Pedernales & Cueva de Marazate & 17.96624 & -71.6355 \\
\hline F58978 & KX355068 & Monophyllus redmani & Dominican Republic & Pedernales & Cueva de Marazate & 17.96624 & -71.6355 \\
\hline F58979 & KX355069 & Monophyllus redmani & Dominican Republic & Pedernales & Cueva de Marazate & 17.96624 & -71.6355 \\
\hline F58998 & KX355070 & Monophyllus redmani & Dominican Republic & & Cueva del Pomier & 18.46698 & -70.1358 \\
\hline F58999 & KX355071 & Monophyllus redmani & Dominican Republic & & Cueva del Pomier & 18.46698 & -70.1358 \\
\hline F59000 & KX355072 & Monophyllus redmani & Dominican Republic & La Vega & Parque Nacional Ar & 19.06727 & -70.8632 \\
\hline F59201 & KX355073 & Monophyllus redmani & Dominican Republic & La Vega & Parque Nacional Ar & 19.06727 & -70.8632 \\
\hline F59221 & KX355074 & Monophyllus redmani & Dominican Republic & Sanchez Ramirez & Cueva Honda de Ju & 19.13234 & -70.0796 \\
\hline F59222 & KX355075 & Monophyllus redmani & Dominican Republic & Sanchez Ramirez & Cueva Honda de Ju & 19.13234 & -70.0796 \\
\hline F59223 & KX355076 & Monophyllus redmani & Dominican Republic & Sanchez Ramirez & Cueva Honda de Ju & 19.13234 & -70.0796 \\
\hline F59228 & KX355077 & Monophyllus redmani & Dominican Republic & Monte Plata & Cueva la Chepa, 14 & 18.86914 & -69.5763 \\
\hline F59229 & KX355078 & Monophyllus redmani & Dominican Republic & Monte Plata & Cueva la Chepa, 14 & 18.86914 & -69.5763 \\
\hline F59230 & KX355079 & Monophyllus redmani & Dominican Republic & Monte Plata & Cueva la Chepa, 14 & 18.86914 & -69.5763 \\
\hline F59254 & KX355080 & Monophyllus redmani & Dominican Republic & El Seibo & $7 \mathrm{~km}$ SE of Miches & 18.9254 & -69.0078 \\
\hline F59306 & KX355081 & Monophyllus redmani & Dominican Republic & La Altagracia & Punta Cana Ecologi & i 18.52531 & -68.3808 \\
\hline F53473 & KT023216 & Monophyllus redmani & JAMAICA & SAINT CATHERII & I ST. CLAIR CAVE, $\varsigma$ & 18.15101 & -77.0909 \\
\hline F53476 & KT023217 & Monophyllus redmani & JAMAICA & SAINT CATHERII & I ST. CLAIR CAVE, ؟ & 18.15101 & -77.0909 \\
\hline F53529 & KT023218 & Monophyllus redmani & JAMAICA & SAINT CATHERII & I ST. CLAIR CAVE, ؟ & 18.15101 & -77.0909 \\
\hline F53530 & KT023219 & Monophyllus redmani & JAMAICA & SAINT CATHERII & I ST. CLAIR CAVE, $\varsigma$ & 18.15101 & -77.0909 \\
\hline F58980 & KX355082 & Mormoops blainvillii & Dominican Republic & Pedernales & Cueva de Marazate & 17.96624 & -71.6355 \\
\hline F58981 & KX355083 & Mormoops blainvillii & Dominican Republic & Pedernales & Cueva de Marazate & 17.96624 & -71.6355 \\
\hline F58982 & KX355084 & Mormoops blainvillii & Dominican Republic & Pedernales & Cueva de Marazate & 17.96624 & -71.6355 \\
\hline F59215 & KX355085 & Mormoops blainvillii & Dominican Republic & Sanchez Ramirez & Cueva Honda de Ju & 19.13234 & -70.0796 \\
\hline F59216 & KX355086 & Mormoops blainvillii & Dominican Republic & Sanchez Ramirez & Cueva Honda de Ju & 19.13234 & -70.0796 \\
\hline F59217 & KX355087 & Mormoops blainvillii & Dominican Republic & Sanchez Ramirez & Cueva Honda de Ju & 19.13234 & -70.0796 \\
\hline F59234 & KX355088 & Mormoops blainvillii & Dominican Republic & Monte Plata & Cueva la Chepa, 14 & 18.86914 & -69.5763 \\
\hline F59235 & KX355089 & Mormoops blainvillii & Dominican Republic & Monte Plata & Cueva la Chepa, 14 & 18.86914 & -69.5763 \\
\hline F59236 & KX355090 & Mormoops blainvillii & Dominican Republic & Monte Plata & Cueva la Chepa, 14 & 18.86914 & -69.5763 \\
\hline F53465 & KT023220 & Mormoops blainvillii & JAMAICA & SAINT CATHERII & I ST. CLAIR CAVE, $\subseteq$ & 18.15101 & -77.0909 \\
\hline F53475 & KT023221 & Mormoops blainvillii & JAMAICA & SAINT CATHERII & I ST. CLAIR CAVE, $\subseteq$ & 18.15101 & -77.0909 \\
\hline F53483 & KT023222 & Mormoops blainvillii & JAMAICA & SAINT ELIZABET & I 1 KM NE OF BALAC & ( 18.18281 & -77.6437 \\
\hline F53484 & KT023223 & Mormoops blainvillii & JAMAICA & SAINT ELIZABET & 1 KM NE OF BALAC & ( 18.18281 & -77.6437 \\
\hline F53487 & KT023224 & Mormoops blainvillii & JAMAICA & SAINT ELIZABET & 4 KM NE OF BALA & ( 18.20687 & -77.6258 \\
\hline F53503 & KT023225 & Mormoops blainvillii & JAMAICA & WESTMORELAN & | 13 KM NW OF BLU & 18.28515 & -78.0549 \\
\hline F53504 & KT023226 & Mormoops blainvillii & JAMAICA & WESTMORELAN & I 13 KM NW OF BLU & 18.28515 & -78.0549 \\
\hline F53510 & KT023227 & Mormoops blainvillii & JAMAICA & WESTMORELAN & | 13.5 KM NW OF BL & 18.29126 & -78.0438 \\
\hline F53543 & KT023228 & Mormoops blainvillii & JAMAICA & SAINT CATHERII & ST. CLAIR CAVE, $\subseteq$ & 18.15101 & -77.0909 \\
\hline F53544 & KT023229 & Mormoops blainvillii & JAMAICA & SAINT CATHERIN & ST. CLAIR CAVE, $\subseteq$ & 18.15101 & -77.0909 \\
\hline F53545 & KT023230 & Mormoops blainvillii & JAMAICA & SAINT CATHERIN & ST. CLAIR CAVE, $\subseteq$ & 18.15101 & -77.0909 \\
\hline F53546 & KT023231 & Mormoops blainvillii & JAMAICA & SAINT CATHERIN & ST. CLAIR CAVE, $\subseteq$ & 18.15101 & -77.0909 \\
\hline F53547 & KT023232 & Mormoops blainvillii & JAMAICA & SAINT CATHERIN & ST. CLAIR CAVE, $\subseteq$ & 18.15101 & -77.0909 \\
\hline
\end{tabular}


F34469 EF080489 BCBN286-05 Myotis albescens F38652 EF080490 BCBNT036-0 Myotis albescens F38792 JF448095 ABGYC242-C Myotis albescens F38840 JF455112 ABGYC261-C Myotis albescens F38957 EF080491 BCBNT103-0 Myotis albescens F44787 JF455110 ABGYE211-C Myotis albescens F44788 JF455111 ABGYE212-C Myotis albescens F45126 EF080484 BCBNT537-0 Myotis albescens F45170 EF080486 BCBNT539-0 Myotis albescens F45177 EF080485 BCBNT540-0 Myotis albescens F45327 EF080492 BCBNT571-0 Myotis albescens F51462 JF455103 ABGYG595-( Myotis albescens F51463 JF455102 ABGYG596-( Myotis albescens F51486 JF455109 ABGYG619-( Myotis albescens F51487 JF455108 ABGYG620-( Myotis albescens F51488 JF455107 ABGYG621-C Myotis albescens F51489 JF455106 ABGYG622-( Myotis albescens F51490 JF455105 ABGYG623-( Myotis albescens F51491 JF455104 ABGYG624-( Myotis albescens F51550 JF455101 ABGYG683-( Myotis albescens FN31019 EF080488 BCBN136-05 Myotis albescens FN31021 JF455113 ABGYA161-C Myotis albescens FN33423 EF080487 BCBN131-05 Myotis albescens F53019 HQ545684 ABSRA998-1 Myotis albescens F53026 HQ545444 ABSRA1005- Myotis albescens F54344 JF448094 ABSRA392-0 Myotis albescens F54364 JF447689 ABSRA412-0 Myotis albescens F54520 EU096807 ABSMS037-C Myotis albescens GU723114 GBMA4445-1 Myotis californicus GU723115 GBMA4444-1 Myotis californicus GU723116 GBMA4443-1 Myotis californicus GU723117 GBMA4442-1 Myotis californicus GU723118 GBMA4441-1 Myotis californicus GU723119 GBMA4440-1 Myotis californicus GU723120 GBMA4439-1 Myotis californicus GU723121 GBMA4438-1 Myotis californicus GU723207 GBMA4352-1 Myotis californicus GU723208 GBMA4351-1 Myotis californicus GU723209 GBMA4350-1 Myotis californicus MyCa03-( GU686085 ABMGH004-( Myotis californicus MyCa05-( GU686086 ABMGH001-( Myotis californicus MyCa05-25 ABMGH002-( Myotis californicus MyCa05-؛ GU686087 ABMGH003-( Myotis californicus MyCa070 HQ919213 ABMGH037-( Myotis californicus 23_MTA MXTOP898-1 Myotis californicus 3120_MD MXBRO039- Myotis californicus 3216 MD MXBRO159-' Myotis californicus 36_MTA MXTOP899-1 Myotis californicus 47-MTA MXTOP897-1 Myotis californicus $70 \overline{7} \_\mathrm{MDL}$ MXTOP896- 1 Myotis californicus

F35471 JF446520 BCBN402-05 Myotis elegans F35497 JF446521 BCBN414-05 Myotis elegans F35527 JF446522 BCBN430-05 Myotis elegans FN31983 JF446807 BCBN209-05 Myotis elegans FN32364 JF448096 BCBN231-05 Myotis elegans GU723122 GBMA4437-1 Myotis evotis GU723123 GBMA4436-1 Myotis evotis GU723124 GBMA4435-1 Myotis evotis GU723126 GBMA4433-1 Myotis evotis GU723213 GBMA4346-1 Myotis evotis GU723214 GBMA4345-1 Myotis evotis GU723215 GBMA4344-1 Myotis evotis 350_ERM HM431990 MXTOP326-( Myotis evotis F35451 JF448097 BCBN394-05 Myotis keays F35530 JF446523 BCBN431-05 Myotis keaysi F35531 JF446524 BCBN432-05 Myotis keaysi F35532 JF446536 ABCSA753-0 Myotis keaysi F35533 JF446535 ABCSA754-0 Myotis keays F35534 JF446533 ABCSA755-0 Myotis keaysi F35535 JF446532 ABCSA756-0 Myotis keaysi F35608 JF446525 BCBN450-05 Myotis keaysi F35609 JF446529 ABCSA812-0 Myotis keaysi F35610 JF446528 ABCSA813-0 Myotis keaysi F35611 JF446527 ABCSA814-0 Myotis keaysi F35643 JF446531 ABCSA839-0 Myotis keaysi F35644 JF446530 ABCSA840-0 Myotis keaysi F35645 JF446537 ABCSA841-0 Myotis keaysi F35702 JF446526 BCBN464-05 Myotis keaysi F35703 JF446534 ABCSA891-0 Myotis keays F34192 JF446810 BCBN276-05 Myotis keays
Guyana

Guyana

Guyana

Guyana

Guyana

Guyana

Guyana

Guyana

Guyana

Guyana

Guyana

Guyana

Guyana

Guyana

Guyana

Guyana

Guyana

Guyana

Guyana

Guyana

Guyana

Guyana

Guyana

Suriname

Suriname

Suriname

Suriname

Suriname
East Berbice-Cor€ Orealla; Orealla Villi 5.317 Upper Takutu-Upr Chodikar River, 55 |1.367 Upper Takutu-Upr Gunn'S Strip $\quad 1.65$ Potaro-Siparuni Iwokrama Reserve, 4.75 Potaro-Siparuni Iwokrama Reserve, 4.75 Potaro-Siparuni Kabukalli Landing, | 4.283 Potaro-Siparuni Kabukalli Landing, I 4.283 Potaro-Siparuni Iwokrama Field Stat 4.667 Potaro-Siparuni Iwokrama Field Stat 4.667 Potaro-Siparuni Iwokrama Field Stat 4.667 Demerara-Mahaic Madewini Creek Bri 6.5 Upper Takutu-Upr Dadanawa Ranch 2.824 Upper Takutu-Upr Dadanawa Ranch 2.824 Upper Takutu-Upr Dadanawa Ranch 2.824 Upper Takutu-Upr Dadanawa Ranch 2.824 Upper Takutu-Upr Dadanawa Ranch 2.824 Upper Takutu-Upr Dadanawa Ranch 2.824 Upper Takutu-Upr Dadanawa Ranch 2.824 Upper Takutu-Upr Dadanawa Ranch 2.824 Upper Takutu-Upr Dadanawa Ranch 2.824

Potaro-Siparuni $40 \mathrm{Km} \mathrm{Ne}$ Of Suram 4.38333 Potaro-Siparuni $40 \mathrm{Km} \mathrm{Ne}$ Of Surar 4.383 Upper Takutu-Upr Karanambo $\quad 3.75$ Sipaliwini Kushere Landing, S 1.944 Sipaliwini Kushere Landing, S 1.944 Nickerie Bakhuis, Transect 94.478 Nickerie Bakhuis, Transect 94.478 Sipaliwini
$-57.333$

$-58.767$

$-58.633$

$-59.017$

$-59.017$

$-58.517$

$-58.517$

$-58.683$

$-58.683$

$-58.683$

$-58.217$

$-59.526$

$-59.526$

$-59.526$

$-59.526$

$-59.526$

$-59.526$

$-59.526$

$-59.526$ $-59.526$

- 8.8667

$-58.867$

$-59.3$

$-56.063$

$-56.063$

$-57.042$

$-57.042$

$-57.178$

\begin{tabular}{|c|c|c|c|}
\hline British Columbia & Moresby Is. & 53.25 & -131.82 \\
\hline British Columbia & Moresby Is. & 53.25 & -131.82 \\
\hline British Columbia & Graham Is. & 53.68 & -132.18 \\
\hline British Columbia & Graham Is. & 53.68 & -132.18 \\
\hline British Columbia & Huxley Is. & 52.44 & -131.36 \\
\hline Baja California Su & & 27.677 & -113.017 \\
\hline Baja California Su & km 9 carretera San & 24.1639 & -110.501 \\
\hline Sonora & Rancho EI Suareno & 30.0639 & -111.019 \\
\hline Baja California Su & & 27.677 & -113.017 \\
\hline Baja California Su & & 27.602 & -113.071 \\
\hline Baja California Su & & 23.633 & -109.683 \\
\hline Ahuachapan & El Imposible, El Ref & $f 13.8$ & -90 \\
\hline Ahuachapan & sible, El Ref & $f 13.8$ & -90 \\
\hline Ahuachapan & El Imposible, El Ref & $f 13.8$ & -90 \\
\hline Peten & 10 Km N Of Tikal & 17.3 & -89.617 \\
\hline Peten & Campo Los Guacan & 17.6 & -90.817 \\
\hline
\end{tabular}

\begin{tabular}{|c|c|c|c|}
\hline Mexico & Baja California S & 26.031 & -111.833 \\
\hline El Salvador & Ahuachapan & El Imposible, San F/13.85 & -90 \\
\hline El Salvador & Santa Ana & Parque Nacional Mc 14.417 & -89.367 \\
\hline El Salvador & Santa Ana & Parque Nacional Mc 14.417 & -89.367 \\
\hline El Salvador & Santa Ana & Parque Nacional Mc 14.417 & -89.367 \\
\hline El Salvador & Santa Ana & Parque Nacional Mc 14.417 & -89.367 \\
\hline EI Salvador & Santa Ana & Parque Nacional Mc 14.417 & -89.367 \\
\hline EI Salvador & Santa Ana & Parque Nacional Mc 14.417 & -89.367 \\
\hline El Salvador & Santa Ana & Parque Nacional Mc 14.417 & -89.367 \\
\hline El Salvador & Santa Ana & Parque Nacional Mc 14.417 & -89.367 \\
\hline El Salvador & Santa Ana & Parque Nacional Mc 14.417 & -89.367 \\
\hline El Salvador & Santa Ana & Parque Nacional Mc 14.417 & -89.367 \\
\hline El Salvador & Santa Ana & Parque Nacional Mc 14.4 & -89.367 \\
\hline El Salvador & Santa Ana & Parque Nacional Mc 14.4 & -89.367 \\
\hline El Salvador & Santa Ana & Parque Nacional Mc 14.4 & -89.367 \\
\hline El Salvador & Santa Ana & Parque Nacional Mc 14.4 & -89.367 \\
\hline El Salvador & Santa Ana & Parque Nacional Mc 14.4 & -89.367 \\
\hline Guatemala & Zacapa & $2 \mathrm{Km} \mathrm{N}$ Of San Lore 15.1 & -90.667 \\
\hline
\end{tabular}


FN31782 JF446808 BCBN177-05 Myotis keaysi FN31783 JF446809 BCBN178-05 Myotis keaysi AVB0803: HM208665 ABVSC022-0 Myotis keaysi AVB0803: HM208666 ABVSC029-0 Myotis keays FN30280 JF447270 BCBN043-05 Myotis keaysi FN30281 JF447271 BCBN044-05 Myotis keaysi FN30435 JF447276 ABMXA760-C Myotis keays FN33841 JF447275 ABMXA932-C Myotis keaysi FN33842 JF447274 ABMXA933-C Myotis keaysi MDE6008 JF447273 ABMXA950-C Myotis keays MDE600气 JF447272 ABMXA951-C Myotis keays F38153 JF447424 BCBN677-05 Myotis keaysi T-4845 ABFG453-10 Myotis martiniquensis T-4848 HQ918456 ABFG456-10 Myotis martiniquensis T-5144 HQ918483 ABFG495-10 Myotis martiniquensis T-5242 ABFG506-10 Myotis martiniquensis T-5243 HQ918490 ABFG507-10 Myotis martiniquensis V-2024: N ABFG453-10 Myotis martiniquensis V-2027: N HQ918456 ABFG456-10 Myotis martiniquensis V-2246 HQ918483 ABFG495-10 Myotis martiniquensis V-2352 ABFG506-10 Myotis martiniquensis V-2353 HQ918490 ABFG507-10 Myotis martiniquensis JN847708 GBMA4859-1Myotis nigricans

F37100 JQ601621 BCBN570-05 Myotis nigricans

F37290 JQ601624 BCBN744-05 Myotis nigricans F37460 JQ601611 ABECA279-0 Myotis nigricans F37461 JQ601612 ABECA280-0 Myotis nigricans F37708 BCBN833-05 Myotis nigricans F40422 JQ601630 BCBN989-05 Myotis nigricans F40423 JQ601631 BCBN990-05 Myotis nigricans F41851 JQ601620 ABECB162-0 Myotis nigricans

F34589 EF080494 BCBN307-05 Myotis nigricans PS1

F38924 JQ601575 ABGYC293-C Myotis nigricans PS1

F46427 JQ601582 ABGYF302-0 Myotis nigricans PS1

FN31020 EF080493 BCBN137-05 Myotis nigricans PS1

F54752 EU096808 ABSMS269-C Myotis nigricans PS1

F36942 JQ601569 ABGYB714-C Myotis nigricans PS2

F36943 JQ601570 ABGYB715-C Myotis nigricans PS2

F36946 JQ601571 ABGYB717-C Myotis nigricans PS2

F38734 JQ601572 ABGYC198-C Myotis nigricans PS2

F38735 JQ601573 ABGYC199-C Myotis nigricans PS2

F38769 JQ601574 ABGYC227-C Myotis nigricans PS2

F43327 JQ601600 BCBNT269-0 Myotis nigricans PS2

F43329 JQ601579 ABGYD053-C Myotis nigricans PS2

F43330 JQ601580 ABGYD054-C Myotis nigricans PS2

F51485 JQ601586 ABGYG618-( Myotis nigricans PS2

F51670 JQ601587 ABGYG803-( Myotis nigricans PS2

F51671 JQ601588 ABGYG804-( Myotis nigricans PS2

F51814 JQ601589 ABGYG947-C Myotis nigricans PS2

F51837 ABGYH035-1 Myotis nigricans PS2

F51838 JQ601590 ABGYG971-( Myotis nigricans PS2

FN33328 JQ601557 ABGYA036-C Myotis nigricans PS2

FN33329 JQ601558 ABGYA037-C Myotis nigricans PS2

FN33330 JQ601559 ABGYA038-C Myotis nigricans PS2

FN33380 JQ601560 ABGYA062-C Myotis nigricans PS2

FN33424 JQ601561 ABGYA089-C Myotis nigricans PS2

FN33432 JQ601562 ABGYA095-C Myotis nigricans PS2

1120_ERI HQ575916 MXTOP895-1 Myotis peninsularis

1375_ERI HQ575866 MXTOP791-1 Myotis peninsularis 1376 ERI HQ575867 MXTOP792-1 Myotis peninsularis 1377 ERI HQ575868 MXTOP793-1 Myotis peninsularis 1378_ERI HQ575869 MXTOP794-1 Myotis peninsularis 1379_ERI HQ575870 MXTOP795-1 Myotis peninsularis 1380 ERI MXTOP796-1 Myotis peninsularis 1381 ERI HQ575871 MXTOP797-1 Myotis peninsularis 1382_ERI HQ575872 MXTOP798-1 Myotis peninsularis 1383_ERI HQ575873 MXTOP799-1 Myotis peninsularis 1384 ER HQ575874 MXTOP800-1 Myotis peninsularis JN847709 GBMA4858-1 Myotis riparius JF442219 GBMA4805-1Myotis riparius JF442223 GBMA4801-1Myotis riparius JF442225 GBMA4799-1Myotis riparius

F37070 JQ601604 ABECA006-0 Myotis riparius

F37085 JQ601605 ABECA018-0 Myotis riparius

F37137 JQ601606 ABECA051-0 Myotis riparius

F37176 JQ601607 ABECA072-0 Myotis riparius

F37191 JQ601608 ABECA083-0 Myotis riparius

F37202 JQ601622 BCBN712-05 Myotis riparius

F37203 JQ601623 BCBN713-05 Myotis riparius

\begin{tabular}{|c|c|c|c|}
\hline Guatemala & Peten & $1.5 \mathrm{Km} \mathrm{S}, 1 \mathrm{Km} \mathrm{W}(16.3$ & -89.3333 \\
\hline Guatemala & Peten & $1.5 \mathrm{Km} \mathrm{S}, 1 \mathrm{Km} \mathrm{W}(16.3$ & -89.3333 \\
\hline Mexico & Campeche & Vicinity of Zoh-Lagu 18.5971 & -89.4222 \\
\hline Mexico & Campeche & Vicinity of Zoh-Lagu 18.5971 & -89.4222 \\
\hline Mexico & Yucatan & Loltun $\quad 20.25$ & -89.4833 \\
\hline Mexico & Yucatan & Loltun $\quad 20.25$ & -89.4833 \\
\hline Mexico & Yucatan & $15 \mathrm{Km}$ S Of Tekax, & \\
\hline Mexico & Quintana Roo & Tulum & \\
\hline Mexico & Quintana Roo & Tulum & \\
\hline Mexico & Quintana Roo & Tulum & \\
\hline Mexico & Quintana Roo & Tulum & \\
\hline Panama & Chiriqui & Ojo De Agua, 2 Km 8.867 & -82.75 \\
\hline Martinique & Morne Rouge & Domaine Estripault & \\
\hline Martinique & Morne Rouge & Domaine Estripault & \\
\hline Martinique & Grand Riviere & Habitation Beausejc & \\
\hline Martinique & Grand Riviere & Habitation Beausejc & \\
\hline Martinique & Grand Riviere & Habitation Beausejc & \\
\hline Martinique & & Domaine Estripault & \\
\hline Martinique & & Domaine Estripault & \\
\hline Martinique & & Habitation Beausejc & \\
\hline Martinique & & Habitation Beausejc & \\
\hline Martinique & & Habitation Beausejc & \\
\hline Ecuador & Napo & Parque Nacional Ya -0.65 & -76.45 \\
\hline Ecuador & Napo & Parque Nacional Ya -0.633 & -76.45 \\
\hline Ecuador & Napo & Parque Nacional Ya -0.633 & -76.45 \\
\hline Ecuador & Napo & Parque Nacional Ya -0.633 & -76.45 \\
\hline Ecuador & Napo & Parque Nacional Ya -0.833 & -76.317 \\
\hline Ecuador & Napo & Parque Nacional Ya -0.65 & -76.45 \\
\hline Ecuador & Napo & Parque Nacional Ya-0.65 & -76.45 \\
\hline Ecuador & & $42 \mathrm{Km} \mathrm{S}, 1 \mathrm{Km} \mathrm{E} \mathrm{O}-0.686$ & -76.432 \\
\hline Panama & Chiriqui & Ojo De Agua, 2 Km 8.867 & -82.75 \\
\hline Guyana & East Berbice-Cor€ & Mapenna River; Ma 5.383 & -57.367 \\
\hline Guyana & Potaro-Siparuni & Iwokrama Reserve, 4.75 & -59.017 \\
\hline Guyana & Potaro-Siparuni & Mount Ayanganna, 5.333 & -59.917 \\
\hline Guyana & Potaro-Siparuni & $40 \mathrm{Km} \mathrm{Ne}$ Of Suran 4.38333 & -58.8667 \\
\hline Suriname & Sipaliwini & Bakhuis, Area 8 Cal 4.542 & -56.933 \\
\hline Guyana & Upper Demerara-I & Tropenbos, $20 \mathrm{Km} \leqslant 5.15$ & -58.7 \\
\hline Guyana & Upper Demerara-I & Tropenbos, $20 \mathrm{Km} \leqslant 5.15$ & -58.7 \\
\hline Guyana & Upper Demerara-I & Tropenbos, $20 \mathrm{Km} \leqslant 5.15$ & -58.7 \\
\hline Guyana & Upper Takutu-Upr & Essequibo River, $7 \mid 1.583$ & -58.633 \\
\hline Guyana & Upper Takutu-Upr & Essequibo River, $7 \mid 1.583$ & -58.633 \\
\hline Guyana & Upper Takutu-Upr & Gunn'S Strip $\quad 1.65$ & -58.633 \\
\hline Guyana & Cuyuni-Mazaruni & Paruima & -61.067 \\
\hline Guyana & Cuyuni-Mazaruni & Paruima & -61.067 \\
\hline Guyana & Cuyuni-Mazaruni & Paruima & -61.067 \\
\hline Guyana & Upper Takutu-Upr & Dadanawa Ranch 2.824 & -59.526 \\
\hline Guyana & Upper Takutu-Upr & $2 \mathrm{Km}$ W Of Unabaitı 2.279 & -59.435 \\
\hline Guyana & Upper Takutu-Upr & $2 \mathrm{Km}$ W Of Unabaitı 2.279 & -59.435 \\
\hline Guyana & Upper Takutu-Upr & $1 \mathrm{Km}$ Upstream Fro 2.486 & -59.476 \\
\hline Guyana & Upper Takutu-Upr & $1 \mathrm{Km}$ Upstream Fro 2.486 & -59.476 \\
\hline Guyana & Upper Takutu-Upr & $1 \mathrm{Km}$ Upstream Fro 2.486 & -59.476 \\
\hline Guyana & Upper Takutu-Upr & Kuma River, 5 Mi E 3.267 & -59.717 \\
\hline Guyana & Upper Takutu-Upr & Kuma River, 5 Mi E 3.267 & -59.717 \\
\hline Guyana & Upper Takutu-Upr & Kuma River, 5 Mi E, 3.267 & -59.717 \\
\hline Guyana & Upper Takutu-Upr & Kuma River, 5 Mi E 3.267 & -59.717 \\
\hline Guyana & Upper Takutu-Upr & Karanambo $\quad 3.75$ & -59.3 \\
\hline Guyana & Upper Takutu-Upr & Karanambo & -59.3 \\
\hline Mexico & Baja California Su & 23.516 & -109.929 \\
\hline Mexico & Baja California Su & 23.174 & -109.611 \\
\hline Mexico & Baja California Su & 23.174 & -109.611 \\
\hline Mexico & Baja California Su & 23.174 & -109.611 \\
\hline Mexico & Baja California Su & 23.174 & -109.611 \\
\hline Mexico & Baja California Su & 23.174 & -109.611 \\
\hline Mexico & Baja California Su & 23.174 & -109.611 \\
\hline Mexico & Baja California Su & 23.174 & -109.611 \\
\hline Mexico & Baja California Su & 23.174 & -109.611 \\
\hline Mexico & Baja California Su & 23.174 & -109.611 \\
\hline Mexico & Baja California Su & 23.174 & -109.611 \\
\hline \multicolumn{4}{|l|}{ Ecuador } \\
\hline \multicolumn{4}{|l|}{ Ecuador } \\
\hline \multicolumn{4}{|l|}{ Ecuador } \\
\hline Ecuador & Napo & Parque Nacional Ya -0.65 & -76.45 \\
\hline Ecuador & Napo & Parque Nacional Ya-0.65 & -76.45 \\
\hline Ecuador & Napo & Parque Nacional Ya -0.683 & -76.433 \\
\hline Ecuador & Napo & Parque Nacional Ya -0.617 & -76.467 \\
\hline Ecuador & Napo & Parque Nacional Ya -0.617 & -76.467 \\
\hline Ecuador & Napo & Parque Nacional Ya -0.683 & -76.433 \\
\hline Ecuador & Napo & Parque Nacional Ya -0.683 & -76.433 \\
\hline
\end{tabular}


F37393 JQ601609 ABECA219-0 Myotis riparius F37452 JQ601610 ABECA273-0 Myotis riparius F37465 JQ601613 ABECA283-0 Myotis riparius F37481 JQ601614 ABECA295-0 Myotis riparius F37524 JQ601625 BCBN796-05 Myotis riparius F37540 JQ601626 BCBN801-05 Myotis riparius F37628 JQ601627 BCBN817-05 Myotis riparius F37629 JQ601628 BCBN818-05 Myotis riparius F37892 JQ601629 BCBN865-05 Myotis riparius F40120 JQ601615 ABECA728-0 Myotis riparius F40162 JQ601616 ABECA760-0 Myotis riparius F40368 JQ601617 ABECA928-0 Myotis riparius F41898 JF444332 ABRMM152-I Myotis riparius F41899 JF444333 ABRMM153-I Myotis riparius F52358 JQ601619 ABECB154-0 Myotis riparius F54195 JQ601618 ABECB081-0 Myotis riparius F38151 JQ601645 BCBN675-05 Myotis riparius F52527 ABGYH082-1 Myotis riparius F53130 HQ919701 ABSRA1108- Myotis riparius F53176 HQ919740 ABSRA1154- Myotis riparius F53307 JQ601201 ABSRA1284- Myotis riparius F53347 JQ601230 ABSRA1323- Myotis riparius F53445 ABSRA1419-Myotis riparius F44007 JQ601554 BCBNT405-0 Myotis riparius PS1 F44024 JQ601555 BCBNT418-0 Myotis riparius PS1 F46545 EF080495 BCBNC043-C Myotis riparius PS1 F51103a JQ601583 ABGYG369-( Myotis riparius PS1 F51173 EF080496 BCBNC187-C Myotis riparius PS1 F51226 EF080497 BCBNC192-C Myotis riparius PS1 F51302 JQ601596 BCBNC198-C Myotis riparius PS1 F51317 JQ601597 BCBNC201-C Myotis riparius PS1 F51330 JQ601598 BCBNC207-C Myotis riparius PS1 F51342 JQ601584 ABGYG570-C Myotis riparius PS1 F51350 JQ601585 ABGYG575-( Myotis riparius PS1 F38029 JQ601635 ABSCA034-0 Myotis riparius PS1 F38030 JQ601639 BCBN606-05 Myotis riparius PS1 F38031 JQ601640 BCBN607-05 Myotis riparius PS1 F39782 JQ601577 ABGYC725-C Myotis riparius PS2 F45234 EF080498 BCBNT552-0 Myotis riparius PS2 F45273 EF080499 BCBNT559-0 Myotis riparius PS2 F45274 EF080500 BCBNT560-0 Myotis riparius PS2 F45307 EF080501 BCBNT569-0 Myotis riparius PS2 F45367 EF080502 BCBNT580-0 Myotis riparius PS2 F50605 JQ601601 BCBNT651-0 Myotis riparius PS2 F50722 BCBNT676-0 Myotis riparius PS2 F50723 EF080503 BCBNT677-0 Myotis riparius PS2

F50744 JQ601602 BCBNT682-0 Myotis riparius PS2 F50790 EF080504 BCBNT693-0 Myotis riparius PS2 F50805 JQ601603 BCBNT697-0 Myotis riparius PS2 F51136 EF080505 BCBNC181-C Myotis riparius PS2 F44411 JQ601550 BCBNT491-0 Myotis riparius PS3 F44430 JQ601551 BCBNT495-0 Myotis riparius PS3 F44438 JQ601546 ABSA100-06 Myotis riparius PS3 F44439 JQ601547 ABSA101-06 Myotis riparius PS3 F44441 JQ601548 ABSA102-06 Myotis riparius PS3 F44449 JQ601647 BCBNT503-0 Myotis riparius PS3 F44453 JQ601552 BCBNT505-0 Myotis riparius PS3 F44461 JQ601549 ABSA115-06 Myotis riparius PS3 F44008 JQ601553 ABSCA130-0 Myotis riparius PS3 F44056 JQ601556 BCBNT446-0 Myotis riparius PS3 F36596 JQ601591 BCBN527-05 Myotis riparius PS3

F36598 JQ601592 BCBN529-05 Myotis riparius PS3 F36599 EF080508 BCBN530-05 Myotis riparius PS3 F36600 JQ601593 BCBN531-05 Myotis riparius PS3 F36601 JQ601564 ABGYB424-C Myotis riparius PS3 F36602 JQ601565 ABGYB425-C Myotis riparius PS3 F36603 JQ601566 ABGYB426-C Myotis riparius PS3 F36612 JQ601567 ABGYB435-C Myotis riparius PS3

F36613 JQ601568 ABGYB436-C Myotis riparius PS3 F38577 JQ601599 BCBNT022-0 Myotis riparius PS3 F38748 EF080509 BCBNT063-0 Myotis riparius PS3 F38770 EF080510 BCBNT068-0 Myotis riparius PS3

F39309 JQ601576 ABGYC453-C Myotis riparius PS3 F43300 JQ601578 ABGYD027-C Myotis riparius PS3 F45178 EF080506 BCBNT541-0 Myotis riparius PS3 F46376 EF080512 BCBNT971-0 Myotis riparius PS3 F46444 EF080513 BCBNC002-C Myotis riparius PS3 F46445 JQ601594 BCBNC003-C Myotis riparius PS3 F46697 EF080514 BCBNC056-C Myotis riparius PS3 F46723 EF080515 BCBNC060-C Myotis riparius PS3

\begin{tabular}{|c|c|c|c|}
\hline Ecuador & Napo & Parque Nacional Ya -0.833 & -76.333 \\
\hline Ecuador & Napo & Parque Nacional Ya -0.65 & -76.45 \\
\hline Ecuador & Napo & Parque Nacional Ya -0.633 & -76.45 \\
\hline Ecuador & Napo & Parque Nacional Ya -0.65 & -76.45 \\
\hline Ecuador & Napo & Parque Nacional Ya -0.683 & -76.433 \\
\hline Ecuador & Napo & Parque Nacional Ya -0.683 & -76.433 \\
\hline Ecuador & Napo & Parque Nacional Ya -0.783 & -76.433 \\
\hline Ecuador & Napo & Parque Nacional Ya -0.783 & -76.433 \\
\hline Ecuador & Napo & Parque Nacional Ya -0.683 & -76.433 \\
\hline Ecuador & Napo & Parque Nacional Ya -0.65 & -76.45 \\
\hline Ecuador & Napo & Parque Nacional Ya -0.65 & -76.45 \\
\hline Ecuador & Napo & Parque Nacional Ya & \\
\hline Ecuador & & Rio Capiron, 46 Km -0.688 & -76.476 \\
\hline Ecuador & & Rio Capiron, 46 Km -0.688 & -76.476 \\
\hline Ecuador & & Yasuni Research St -0.837 & -76.334 \\
\hline Ecuador & & Onkone Gare, $38 \mathrm{KI}-0.657$ & -76.454 \\
\hline Panama & Chiriqui & Ojo De Agua, 2 Km 8.867 & -82.75 \\
\hline Suriname & Paramaribo & Tafelberg, Augustus 3.93 & -56.19 \\
\hline Suriname & Sipaliwini & Iconja Landing, Sip c 1.994 & -56.092 \\
\hline Suriname & Sipaliwini & Iconja Landing, Sip̌ 1.994 & -56.092 \\
\hline Suriname & Sipaliwini & Kutari River Camp 2.17526 & -56.788 \\
\hline Suriname & Sipaliwini & Sipaliwini River Can 2.289 & -56.607 \\
\hline Suriname & Sipaliwini & Werehpai Camp 2.363 & -56.698 \\
\hline Costa Rica & Limon & Tortuga Lodge, Tort 10.567 & -83.517 \\
\hline Costa Rica & Limon & Cano Palma Biologi 10.6 & -83.533 \\
\hline Guyana & Potaro-Siparuni & Mount Ayanganna, 5.383 & -59.967 \\
\hline Guyana & Potaro-Siparuni & Kaieteur National P: 5.166 & -59.493 \\
\hline Guyana & Potaro-Siparuni & Kaieteur National P: 5.25 & -59.614 \\
\hline Guyana & Potaro-Siparuni & Kaieteur National P:5.25 & -59.614 \\
\hline Guyana & Potaro-Siparuni & Kaieteur National P:5.25 & -59.614 \\
\hline Guyana & Potaro-Siparuni & Kaieteur National P: 5.25 & -59.614 \\
\hline Guyana & Potaro-Siparuni & Kaieteur National P: 5.25 & -59.614 \\
\hline Guyana & Potaro-Siparuni & Kaieteur National P: 5.175 & -59.481 \\
\hline Guyana & Potaro-Siparuni & Kaieteur National P: 5.175 & -59.481 \\
\hline Panama & Canal Zone & Gamboa $\quad 9.1$ & -79.7 \\
\hline Panama & Canal Zone & Gamboa & -79.7 \\
\hline Panama & Canal Zone & Gamboa & -79.7 \\
\hline Guyana & Cuyuni-Mazaruni & Kamarang $\quad 5.867$ & -60.617 \\
\hline Guyana & Demerara-Mahaic & Ceiba Biological St $\varepsilon 6.5$ & -58.217 \\
\hline Guyana & Demerara-Mahaic & Ceiba Biological St $=6.5$ & -58.217 \\
\hline Guyana & Demerara-Mahaic & Ceiba Biological St $=6.5$ & -58.217 \\
\hline Guyana & Demerara-Mahaic & Ceiba Biological St $=6.5$ & -58.217 \\
\hline Guyana & Demerara-Mahaic & Ceiba Biological St $=6.5$ & -58.217 \\
\hline Guyana & Demerara-Mahaic & Ceiba Biological Ce 6.5 & -58.217 \\
\hline Guyana & Demerara-Mahaic & Ceiba Biological Ce 6.5 & -58.217 \\
\hline Guyana & Demerara-Mahaic & Ceiba Biological Ce 6.5 & -58.217 \\
\hline Guyana & Demerara-Mahaic & Ceiba Biological Ce 6.5 & -58.217 \\
\hline Guyana & Demerara-Mahaic & Ceiba Biological Ce 6.5 & -58.217 \\
\hline Guyana & Demerara-Mahaic & Ceiba Biological Ce 6.5 & -58.217 \\
\hline Guyana & Potaro-Siparuni & Kaieteur National P: 5.25 & -59.614 \\
\hline Brazil & Sao Paulo & Caetetus Ecological-22.383 & -49.667 \\
\hline Brazil & Sao Paulo & Caetetus Ecological-22.383 & -49.667 \\
\hline Brazil & Sao Paulo & Caetetus Ecological -22.383 & -49.667 \\
\hline Brazil & Sao Paulo & Caetetus Ecological -22.383 & -49.667 \\
\hline Brazil & Sao Paulo & Caetetus Ecological -22.383 & -49.667 \\
\hline Brazil & Sao Paulo & Caetetus Ecological -22.383 & -49.667 \\
\hline Brazil & Sao Paulo & Caetetus Ecological -22.383 & -49.667 \\
\hline Brazil & Sao Paulo & Caetetus Ecological -22.383 & -49.667 \\
\hline Costa Rica & Limon & Tortuga Lodge, Tort 10.567 & -83.517 \\
\hline Costa Rica & Limon & Cano Palma Biologi 10.6 & -83.533 \\
\hline Guyana & Upper Takutu-Upr & $5 \mathrm{Km}$ Se Of Suramє 4.1 & -59.05 \\
\hline Guyana & Upper Takutu-Upr & $5 \mathrm{Km}$ Se Of Surame 4.1 & -59.05 \\
\hline Guyana & Upper Takutu-Upr & $5 \mathrm{Km}$ Se Of Surame 4.1 & -59.05 \\
\hline Guyana & Upper Takutu-Upp & $5 \mathrm{Km}$ Se Of Surame 4.1 & -59.05 \\
\hline Guyana & Upper Takutu-Upr & $5 \mathrm{Km}$ Se Of Surame 4.1 & -59.05 \\
\hline Guyana & Upper Takutu-Upr & $5 \mathrm{Km}$ Se Of Surame 4.1 & -59.05 \\
\hline Guyana & Upper Takutu-Upr & $5 \mathrm{Km}$ Se Of Surame 4.1 & -59.05 \\
\hline Guyana & Upper Takutu-Upr & $5 \mathrm{Km}$ Se Of Surame 4.1 & -59.05 \\
\hline Guyana & Upper Takutu-Upr & $5 \mathrm{Km}$ Se Of Surame 4.1 & -59.05 \\
\hline Guyana & Upper Takutu-Upr & Chodikar River, 55 | 1.367 & -58.767 \\
\hline Guyana & Upper Takutu-Upp & Essequibo River, $7 \mid 1.583$ & -58.633 \\
\hline Guyana & Upper Takutu-Upp & Gunn'S Strip $\quad 1.65$ & -58.633 \\
\hline Guyana & Potaro-Siparuni & Iwokrama Reserve, 4.733 & -58.85 \\
\hline Guyana & Cuyuni-Mazaruni & Namai Creek, 5 Km 5.8 & -61.1 \\
\hline Guyana & Potaro-Siparuni & Iwokrama Field Stat 4.667 & -58.683 \\
\hline Guyana & Potaro-Siparuni & Mount Ayanganna, 5.333 & -59.917 \\
\hline Guyana & Potaro-Siparuni & Mount Ayanganna, 5.333 & -59.95 \\
\hline Guyana & Potaro-Siparuni & Mount Ayanganna, 5.333 & -59.95 \\
\hline Guyana & Cuyuni-Mazaruni & Mount Roraima, Rid 5.333 & -60.767 \\
\hline Guyana & Cuyuni-Mazaruni & Mount Roraima, Rid 5.333 & -60.767 \\
\hline
\end{tabular}




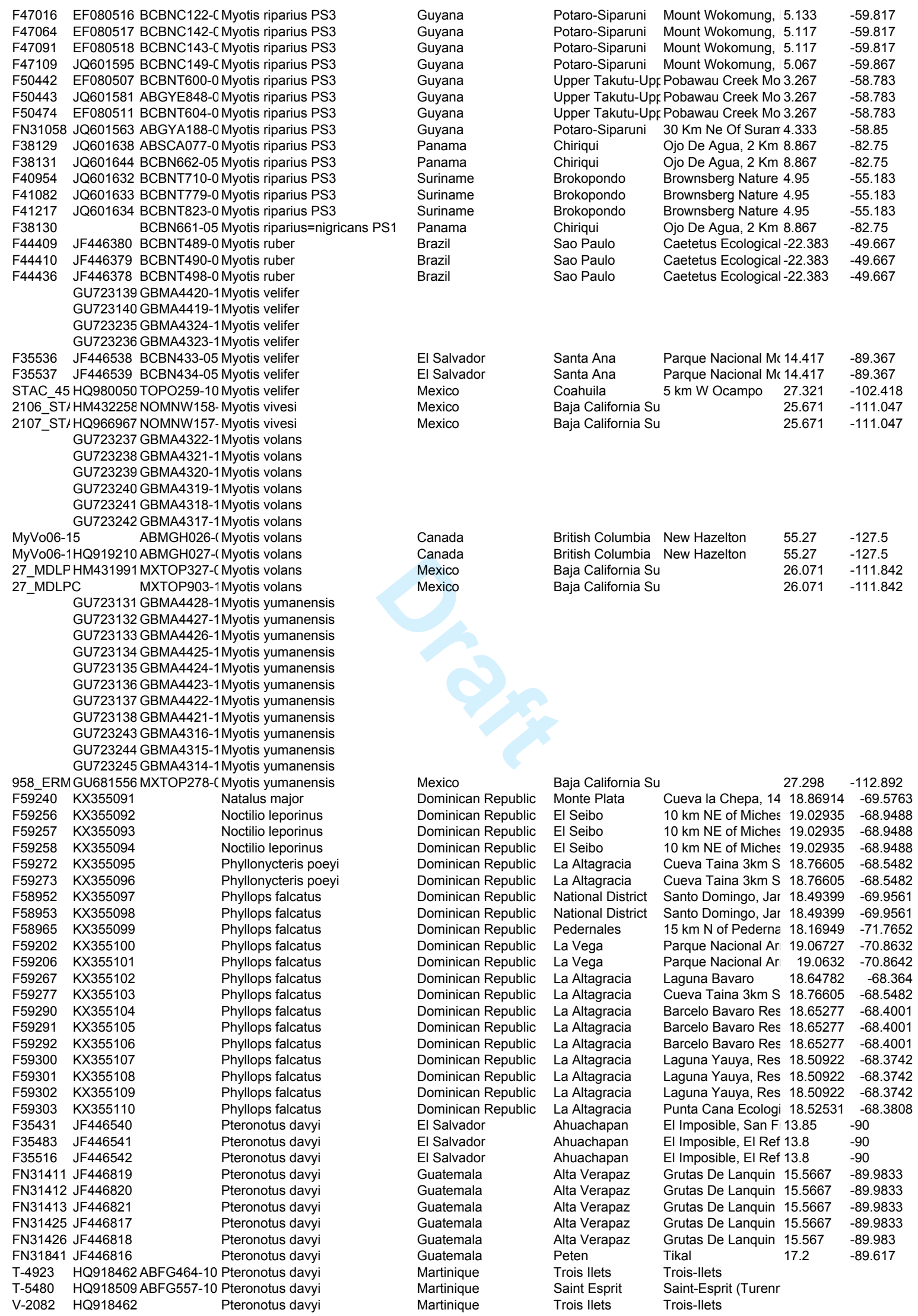




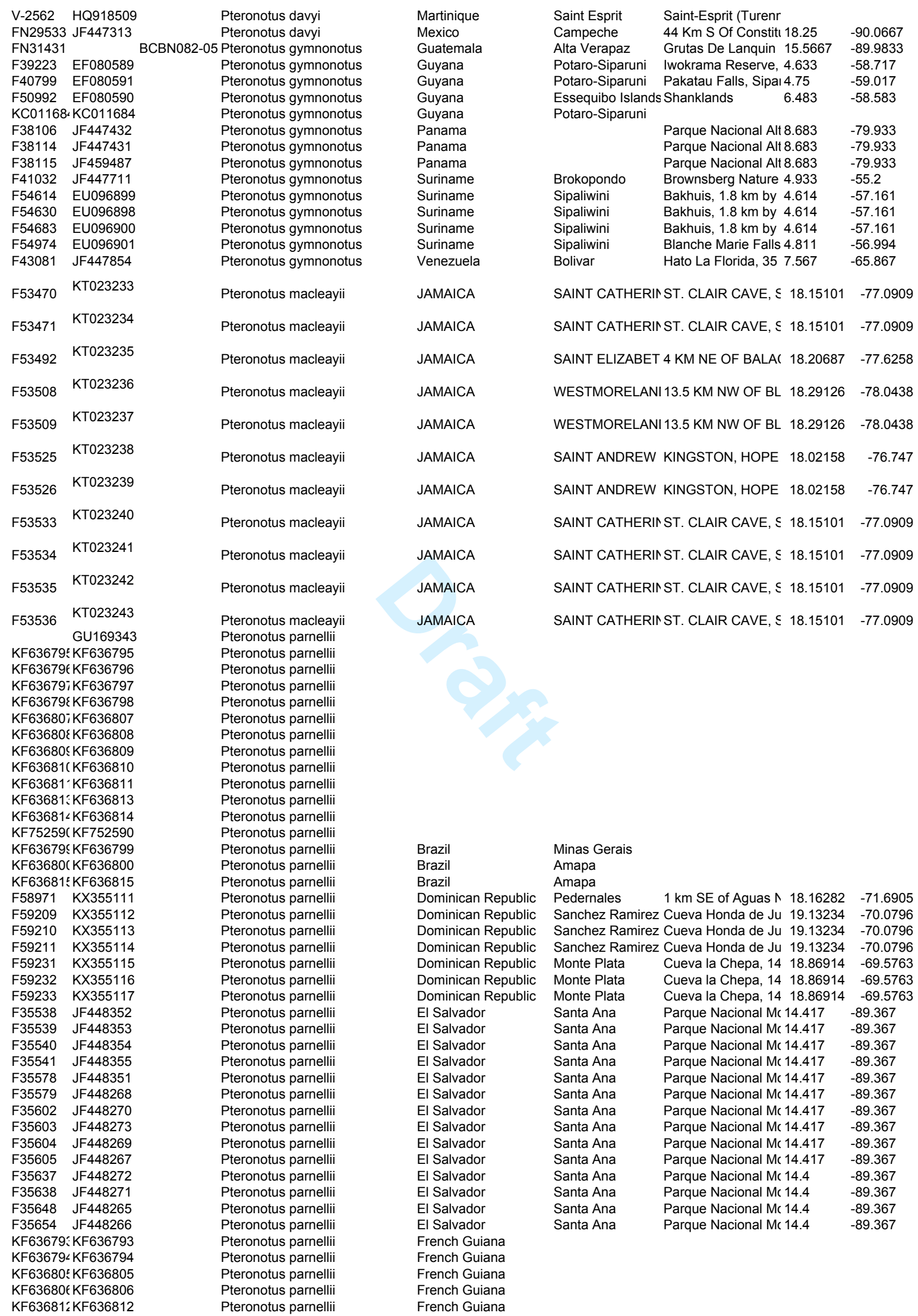


FN31472 JF448362 FN31784 JF448278 FN31785 JF448277 FN31939 JF448276 FN31940 JF448361 FN32279 JF448356 FN32280 JF448357 FN32324 JF448274 FN32343 JF448275

FN32344 JF448358 FN32345 JF448359 FN32371 JF448360 F34480 JF448225

F34481 JF448226

F34492 JF448227

F34519 JF448228

F34520 JF448229

F34521 JF448230

F34522 JF448231

F34544 JF448232

F34545 JF448233

F34624 JF448234

F34625 JF448235

F34626 JF448236

F34627 JF448237

F34628 JF448238

F34629 JF448239

F34630 JF448240

F34631 JF448241

F34632 JF448242

F34633 JF448243

F34634 JF448244

F34635 JF448245

F34636 JF448218

F34637 JF448219

F34638 JF448220

F34639 JF448221

F34640 JF448222

F34641 JF448223

F34642 JF448224

F34658 JF448246

F34668 JF448247

F34669 JF448248

F34670 JF448249

F34671 JF448250

F34672 JF448251

F34701 JF448252

F34702 JF448253

F34703 JF448254

F34856 JF448255

F34869 JF448256

F34946 JF448257

F35044 JF448375

F36357 JF448386

F36358 JF448387

F36359 JF448388

F36392 JF448389

F36393 JF448390

F36436 JF448391

F36453 JF448392

F36454 JF448393

F36469 JF448400

F36546 JF448401

F36547 JF448402

F36548 JF448403

F36589 JF448404

F36678 JF448405

F36837 JF448376

F36838 JF448377

F36839 JF448378

F36883 JF448379

F36884 JF448380

F36885 JF448381

F36929 JF448382

F36934 JF448383

F36956 JF448384

F36957 JF448385

F38266 JF448406

F38295 JF448407

F38296 JF448408
Pteronotus parnellii

Pteronotus parnellii

Pteronotus parnellii

Pteronotus parnelli

Pteronotus parnellii

Pteronotus parnelli

Pteronotus parnelli

Pteronotus parnelli

Pteronotus parnellii

Pteronotus parnellii

Pteronotus parnelli

Pteronotus parnelli

Pteronotus parnellii

Pteronotus parnelli

Pteronotus parnellii

Pteronotus parnelli

Pteronotus parnellii

Pteronotus parnelli

Pteronotus parnelli

Pteronotus parnelli

Pteronotus parnellii

Pteronotus parnelli

Pteronotus parnelli

Pteronotus parnellii

Pteronotus parnellii

Pteronotus parnelli

Pteronotus parnelli

Pteronotus parnellii

Pteronotus parnelli

Pteronotus parnelli

Pteronotus parnelli

Pteronotus parnellii

Pteronotus parnelli

Pteronotus parnelli

Pteronotus parnelli

Pteronotus parnellii

Pteronotus parnellii

Pteronotus parnellii

Pteronotus parnellii

Pteronotus parnelli

Pteronotus parnelli

Pteronotus parnelli

Pteronotus parnellii

Pteronotus parnelli

Pteronotus parnelli

Pteronotus parnellii

Pteronotus parnelli

Pteronotus parnelli

Pteronotus parnelli

Pteronotus parnellii

Pteronotus parnellii

Pteronotus parnellii

Pteronotus parnelli

Pteronotus parnelli

Pteronotus parnelli

Pteronotus parnellii

Pteronotus parnelli

Pteronotus parnellii

Pteronotus parnelli

Pteronotus parnellii

Pteronotus parnelli

Pteronotus parnelli

Pteronotus parnellii

Pteronotus parnelli

Pteronotus parnellii

Pteronotus parnelli

Pteronotus parnelli

Pteronotus parnelli

Pteronotus parnellii

Pteronotus parnelli

Pteronotus parnellii

Pteronotus parnelli

Pteronotus parnelli

Pteronotus parnelli

Pteronotus parnellii

Pteronotus parnelli

Pteronotus parnelli

Pteronotus parnellii

Pteronotus parnelli

Pteronotus parnellii

$\begin{array}{ll}\text { Guatemala } & \text { El Progreso } \\ \text { Guatemala } & \text { Peten } \\ \text { Guatemala } & \text { Peten } \\ \text { Guatemala } & \text { Peten } \\ \text { Guatemala } & \text { Peten } \\ \text { Guatemala } & \text { Peten } \\ \text { Guatemala } & \text { Peten } \\ \text { Guatemala } & \text { Peten } \\ \text { Guatemala } & \text { Peten } \\ \text { Guatemala } & \text { Peten } \\ \text { Guatemala } & \text { Peten }\end{array}$

Rio Uyus, 5 Km E C 14.95

$1.5 \mathrm{Km} \mathrm{S}, 1 \mathrm{Km} \mathrm{W}(16.3$

$1.5 \mathrm{Km} \mathrm{S}, 1 \mathrm{Km} \mathrm{W}(16.3$

$10 \mathrm{Km} \mathrm{N}$ Of Tikal $\quad 17.3$

$10 \mathrm{Km} N$ Of Tikal $\quad 17.3$

El Remate $\quad 17$

El Remate $\quad 17$

Campo Los Guacan 17.6

Campo Los Guacan 17.6

Campo Los Guacan 17.6

Campo Los Guacan 17.6

Campo Los Guacan 17.6

East Berbice-Corє Mapenna River; Ma 5.383

Guyana

Guyana

Guyana

Guyana

Guyana

Guyana

Guyana

Guyana

Guyana

Guyana

Guyana

Guyana

Guyana

Guyana

Guyana

Guyana

Guyana

Guyana

Guyana

Guyana

Guyana

Guyana

Guyana

Guyana

Guyana

Guyana

Guyana

Guyana

Guyana

Guyana

Guyana

Guyana

Guyana

Guyana

Guyana

Guyana

Guyana

Guyana

Guyana

Guyana

Guyana

Guyana

Guyana

Guyana

Guyana

Guyana

Guyana

Guyana

Guyana

Guyana

Guyana

Guyana

Guyana

Guyana

Guyana

Guyana

Guyana

Guyana

Guyana

Guyana

Guyana

Guyana

Guyana

Guyana

Guyana

Guyana

Guyana

Guyana

East Berbice-Cor $€$ Mapenna River; Ma 5.383

East Berbice-Cor€ Mapenna River; Ma 5.383

East Berbice-Corє Mapenna River; Ma 5.383

East Berbice-Corє Mapenna River; Ma 5.383

East Berbice-Cor $€$ Mapenna River; Ma 5.383

East Berbice-Corє Mapenna River; Ma 5.383

East Berbice-Corє Mapenna River; Ma 5.383

East Berbice-Corє Mapenna River; Ma 5.383

East Berbice-Cor€ Mango Landing, Co 5.167

East Berbice-Corє Mango Landing, Co 5.167 East Berbice-Corє Mango Landing, Co 5.167

East Berbice-Cor $\in$ Mango Landing, Co 5.167 East Berbice-Cor $€$ Mango Landing, Co 5.167 East Berbice-Core Mango Landing, Co 5.167 East Berbice-Corє Mango Landing, Co 5.167 East Berbice-Corє Mango Landing, Co 5.167 East Berbice-Cor $\in$ Mango Landing, Co 5.167 East Berbice-Cor $€$ Mango Landing, Co 5.167 East Berbice-Cor $\in$ Mango Landing, Co 5.167 East Berbice-Corє Mango Landing, Co 5.167 East Berbice-Corє Mango Landing, Co 5.167 East Berbice-Cor $\in$ Mango Landing, Co 5.167 East Berbice-Cor $€$ Mango Landing, Co 5.167 East Berbice-Corє Mango Landing, Co 5.167 East Berbice-Core Mango Landing, Co 5.167 East Berbice-Corє Mango Landing, Co 5.167 East Berbice-Cor $€$ Mango Landing, Co 5.167 East Berbice-Corє Mango Landing, Co 5.167 East Berbice-Corє Mango Landing, Co 5.167

East Berbice-Corє Mango Landing, Co 5.167 East Berbice-Core Mango Landing, Co 5.167 East Berbice-Cor€ Mango Landing, Co 5.167 East Berbice-Corє Mango Landing, Co 5.167

East Berbice-Cor $\in$ Mango Landing, Co 5.167 East Berbice-Cor $\in$ Mango Landing, Co 5.167 East Berbice-Corє Mango Landing, Co 5.167 Barima-Waini Baramita, Old Work 7.367

Barima-Waini Baramita, Old Work 7.367 Barima-Waini Baramita, Old Worlc 7.367 Barima-Waini Baramita, Old Worlc 7.367 Upper Takutu-Upr $5 \mathrm{Km}$ Se Of Surame 4.1

Upper Takutu-Upr $5 \mathrm{Km}$ Se Of Surame 4.1 Upper Takutu-Upr $5 \mathrm{Km}$ Se Of Surame 4.1 Upper Takutu-Upr $5 \mathrm{Km}$ Se Of Surame 4.1 Upper Takutu-Upr $5 \mathrm{Km}$ Se Of Surame 4.1 Upper Takutu-Upr $5 \mathrm{Km}$ Se Of Surame 4.1 Upper Takutu-Upr $5 \mathrm{Km}$ Se Of Surame 4.1 Upper Takutu-Upr $5 \mathrm{Km}$ Se Of Surame 4.1 Upper Takutu-Upr $5 \mathrm{Km}$ Se Of Surame 4.1 


\begin{tabular}{|c|c|}
\hline F38297 & JF448409 \\
\hline F38328 & JF448410 \\
\hline F38361 & JF448411 \\
\hline F38370 & JF448286 \\
\hline F38389 & JF448287 \\
\hline F38410 & JF448288 \\
\hline F38552 & JF448289 \\
\hline F38572 & JF448290 \\
\hline F38582 & JF448291 \\
\hline F38656 & JF448292 \\
\hline F38664 & JF448293 \\
\hline F38738 & JF448294 \\
\hline F38773 & JF448295 \\
\hline F38811 & JF448296 \\
\hline F38812 & JF448297 \\
\hline F38815 & JF448298 \\
\hline F38838 & JF448299 \\
\hline F38839 & JF448300 \\
\hline F38870 & JF448301 \\
\hline F38963 & JF448302 \\
\hline F39024 & JF448303 \\
\hline F39025 & JF448363 \\
\hline F39057 & JF448304 \\
\hline F39060 & JF448305 \\
\hline F39130 & JF448306 \\
\hline F39166 & JF448307 \\
\hline F39187 & JF448308 \\
\hline F39190 & JF448309 \\
\hline F39191 & JF448310 \\
\hline F39192 & JF448314 \\
\hline F39201 & JF448315 \\
\hline F39202 & JF448316 \\
\hline F39226 & JF448317 \\
\hline F39227 & JF448318 \\
\hline F39228 & JF448319 \\
\hline F39301 & JF448320 \\
\hline F39303 & JF448321 \\
\hline F39305 & JF448322 \\
\hline F39306 & JF448323 \\
\hline F39427 & JF448324 \\
\hline F39448 & JF448325 \\
\hline F39469 & JF448326 \\
\hline F39471 & JF448327 \\
\hline F39473 & JF448328 \\
\hline F39474 & JF448329 \\
\hline F39756 & JF448330 \\
\hline F40561 & JF448463 \\
\hline F40570 & JF448466 \\
\hline F40838 & JF448417 \\
\hline F40860 & JF448418 \\
\hline F40869 & JF448419 \\
\hline F40870 & JF448420 \\
\hline F43237 & JF448311 \\
\hline F43250 & JF448312 \\
\hline F43251 & JF448313 \\
\hline F43282 & JF448460 \\
\hline F43287 & JF448461 \\
\hline F43557 & JF448467 \\
\hline F43881 & JF448462 \\
\hline F43895 & JF448118 \\
\hline F43896 & EF080592 \\
\hline F43932 & EF080593 \\
\hline F43936 & JF448117 \\
\hline F43951 & JF448116 \\
\hline F43965 & JF448464 \\
\hline F43966 & JF448465 \\
\hline F44663 & JF448421 \\
\hline F44670 & JF448422 \\
\hline F44685 & JF448423 \\
\hline F44698 & JF448431 \\
\hline F44725 & JF448432 \\
\hline F44793 & JF448433 \\
\hline F44823 & JF448434 \\
\hline F44928 & JF448435 \\
\hline F44943 & JF448436 \\
\hline F44975 & JF448437 \\
\hline F44976 & JF448438 \\
\hline F44977 & JF448439 \\
\hline F45037 & JF448440 \\
\hline F45066 & JF448441 \\
\hline
\end{tabular}

Pteronotus parnellii

Pteronotus parnellii

Pteronotus parnellii

Pteronotus parnellii

Pteronotus parnellii

Pteronotus parnellii

Pteronotus parnellii

Pteronotus parnellii

Pteronotus parnellii

Pteronotus parnellii

Pteronotus parnellii

Pteronotus parnellii

Pteronotus parnellii

Pteronotus parnellii

Pteronotus parnellii

Pteronotus parnellii

Pteronotus parnellii

Pteronotus parnellii

Pteronotus parnellii

Pteronotus parnellii

Pteronotus parnellii

Pteronotus parnellii

Pteronotus parnelli

Pteronotus parnellii

Pteronotus parnellii

Pteronotus parnellii

Pteronotus parnellii

Pteronotus parnellii

Pteronotus parnellii

Pteronotus parnellii

Pteronotus parnellii

Pteronotus parnelli

Pteronotus parnelli

Pteronotus parnellii

Pteronotus parnellii

Pteronotus parnellii

Pteronotus parnellii

Pteronotus parnellii

Pteronotus parnellii

Pteronotus parnellii

Pteronotus parnellii

Pteronotus parnelli

Pteronotus parnelli

Pteronotus parnellii

Pteronotus parnellii

Pteronotus parnellii

Pteronotus parnellii

Pteronotus parnellii

Pteronotus parnellii

Pteronotus parnellii

Pteronotus parnellii

Pteronotus parnellii

Pteronotus parnelli

Pteronotus parnellii

Pteronotus parnellii

Pteronotus parnellii

Pteronotus parnelli

Pteronotus parnellii

Pteronotus parnelli

Pteronotus parnelli

Pteronotus parnelli

Pteronotus parnelli

Pteronotus parnelli

Pteronotus parnelli

Pteronotus parnellii

Pteronotus parnelli

Pteronotus parnellii

Pteronotus parnelli

Pteronotus parnellii

Pteronotus parnelli

Pteronotus parnellii

Pteronotus parnellii

Pteronotus parnelli

Pteronotus parnellii

Pteronotus parnellii

Pteronotus parnelli

Pteronotus parnellii

Pteronotus parnellii

Pteronotus parnellii

Pteronotus parnellii
Guyana

Guyana

Guyana

Guyana

Guyana

Guyana

Guyana

Guyana

Guyana

Guyana

Guyana

Guyana

Guyana

Guyana

Guyana

Guyana

Guyana

Guyana

Guyana

Guyana

Guyana

Guyana

Guyana

Guyana

Guyana

Guyana

Guyana

Guyana

Guyana

Guyana

Guyana

Guyana

Guyana

Guyana

Guyana

Guyana

Guyana

Guyana

Guyana

Guyana

Guyana

Guyana

Guyana

Guyana

Guyana

Guyana

Guyana

Guyana

Guyana

Guyana

Guyana

Guyana

Guyana

Guyana

Guyana

Guyana

Guyana

Guyana

Guyana

Guyana

Guyana

Guyana

Guyana

Guyana

Guyana

Guyana

Guyana

Guyana

Guyana

Guyana

Guyana

Guyana

Guyana

Guyana

Guyana

Guyana

Guyana

Guyana

Guyana

Guyana
Potaro-Siparuni Iwokrama Reserve, 4.467 Potaro-Siparuni Iwokrama Reserve, 4.467 Potaro-Siparuni Iwokrama Reserve, 4.667 Potaro-Siparuni Iwokrama Reserve, 4.667 Potaro-Siparuni Iwokrama Reserve, 4.583

Potaro-Siparuni Iwokrama Reserve, 4.583 Upper Takutu-Upr Gunn'S Strip $\quad 1.65$ Upper Takutu-Upr Chodikar River, 55 ।1.367 Upper Takutu-Upr Chodikar River, 55 \$1.367 Upper Takutu-Upr Kamoa River, 50 Kr 1.533 Upper Takutu-Upr Kamoa River, 50 Kr 1.533 Upper Takutu-Upr Essequibo River, $7 \mid 1.583$ Upper Takutu-Upr Gunn'S Strip $\quad 1.65$

Potaro-Siparuni Iwokrama Reserve, 4.75 Potaro-Siparuni Iwokrama Reserve, 4.75 Potaro-Siparuni Iwokrama Reserve, 4.75

Potaro-Siparuni Iwokrama Reserve, 4.75

Potaro-Siparuni Iwokrama Reserve, 4.75

Potaro-Siparuni Iwokrama Reserve, 4.75

Potaro-Siparuni Iwokrama Reserve, 4.75

Potaro-Siparuni Iwokrama Reserve, 4.633

Potaro-Siparuni Iwokrama Reserve, 4.633

Potaro-Siparuni Iwokrama Reserve, 4.633

Potaro-Siparuni Iwokrama Reserve, 4.633

Potaro-Siparuni Iwokrama Reserve, 4.633

Potaro-Siparuni Iwokrama Reserve, 4.633

Potaro-Siparuni Iwokrama Reserve, 4.633

Potaro-Siparuni Iwokrama Reserve, 4.633

Potaro-Siparuni Iwokrama Reserve, 4.633

Potaro-Siparuni Iwokrama Reserve, 4.633

Potaro-Siparuni Iwokrama Reserve, 4.633

Potaro-Siparuni Iwokrama Reserve, 4.633

Potaro-Siparuni Iwokrama Reserve, 4.633

Potaro-Siparuni Iwokrama Reserve, 4.633

Potaro-Siparuni Iwokrama Reserve, 4.633

Potaro-Siparuni Iwokrama Reserve, 4.733

Potaro-Siparuni Iwokrama Reserve, 4.733

Potaro-Siparuni Iwokrama Reserve, 4.733

Potaro-Siparuni Iwokrama Reserve, 4.733

Potaro-Siparuni Iwokrama Reserve, 4.733

Potaro-Siparuni Iwokrama Reserve, 4.733

Potaro-Siparuni Iwokrama Reserve, 4.733

Potaro-Siparuni Iwokrama Reserve, 4.733

Potaro-Siparuni Iwokrama Reserve, 4.733

Potaro-Siparuni Iwokrama Reserve, 4.733

Cuyuni-Mazaruni Kuwaima Fall, Mazć 6.05

Potaro-Siparuni Turtle Mountain, 104.733

Potaro-Siparuni Turtle Mountain, 104.733

Potaro-Siparuni Pakatau Falls, Sipal 4.75

Potaro-Siparuni Pakatau Falls, Sipal 4.75

Potaro-Siparuni Pakatau Falls, Sipal 4.75

Potaro-Siparuni Pakatau Falls, Sipal 4.75

Cuyuni-Mazaruni Namai Creek, 5 Km 5.8

Cuyuni-Mazaruni Namai Creek, $5 \mathrm{Km} 5.8$

Cuyuni-Mazaruni Namai Creek, $5 \mathrm{Km} 5.8$

Cuyuni-Mazaruni Namai Creek, 5 Km 5.8

Cuyuni-Mazaruni Namai Creek, 5 Km 5.8

Potaro-Siparuni Cow Fly Camp, 40 \&.333

Potaro-Siparuni Turtle Mountain, 104.733

Potaro-Siparuni Turtle Mountain, 104.733

Potaro-Siparuni Turtle Mountain, 104.733

Potaro-Siparuni Turtle Mountain, 104.73

Potaro-Siparuni Turtle Mountain, 104.733

Potaro-Siparuni Turtle Mountain, 104.733

Potaro-Siparuni Turtle Mountain, 104.733

Potaro-Siparuni Turtle Mountain, 104

Potaro-Siparuni Kabukalli Landing, I 4.283

Potaro-Siparuni Kabukalli Landing, | 4.283

Potaro-Siparuni Kabukalli Landing, 14.283

Potaro-Siparuni Kabukalli Landing, | 4.283

Potaro-Siparuni Kabukalli Landing, | 4.283

Potaro-Siparuni Kabukalli Landing, | 4.283

Potaro-Siparuni Kabukalli Landing, 14.283

Potaro-Siparuni Sand Stone, Iwokra 4.383

Potaro-Siparuni Sand Stone, Iwokra 4.383

Potaro-Siparuni Mount Daniel Cutlin 4.45

Potaro-Siparuni Mount Daniel Cutlin 4.45

Potaro-Siparuni Mount Daniel Cutlin 4.45

Potaro-Siparuni Mount Daniel Cutlin 4.45

Potaro-Siparuni Mount Daniel Cutlin 4.45

$-58.783$

$-58.783$

$-58.683$

$-58.683$

$-58.617$

$-58.617$

$-58.633$

$-58.767$

$-58.767$

$-58.833$

$-58.833$

$-58.633$

59.017

$-59.017$

$-59.017$

(59.017

59.017

$-59.017$

$-59.017$

$-58.717$

$-58.717$

$-58.717$

$-58.717$

$-58.717$

$-58.717$

$-58.717$

-

$-58.717$

$-58.717$

$-58.717$

- 8.717

$-58.717$

$-58.717$

$-58.717$

$-58.85$

$-58.85$

$-58.85$

$-58.85$

$-58.85$

$-58.85$

$-58.85$

$-58.85$

$-58.85$

$-58.85$

$-60.65$

$-58.717$ 
Pteronotus parnellii Pteronotus parnelli Pteronotus parnellii Pteronotus parnellii Pteronotus parnelli Pteronotus parnelli Pteronotus parnellii Pteronotus parnelli Pteronotus parnelli Pteronotus parnelli Pteronotus parnelli Pteronotus parnellii Pteronotus parnelli Pteronotus parnelli Pteronotus parnelli Pteronotus parnelli Pteronotus parnellii Pteronotus parnelli Pteronotus parnellii Pteronotus parnelli Pteronotus parnelli Pteronotus parnellii Pteronotus parnelli Pteronotus parnelli Pteronotus parnelli Pteronotus parnellii Pteronotus parnelli Pteronotus parnellii Pteronotus parnellii Pteronotus parnellii Pteronotus parnelli Pteronotus parnelli Pteronotus parnellii Pteronotus parnelli Pteronotus parnellii Pteronotus parnelli Pteronotus parnelli Pteronotus parnelli Pteronotus parnellii Pteronotus parnelli Pteronotus parnellii Pteronotus parnellii Pteronotus parnelli Pteronotus parnellii Pteronotus parnellii Pteronotus parnelli Pteronotus parnelli Pteronotus parnelli Pteronotus parnelli Pteronotus parnellii Pteronotus parnelli Pteronotus parnellii Pteronotus parnelli Pteronotus parnellii Pteronotus parnellii Pteronotus parnellii Pteronotus parnelli Pteronotus parnellii Pteronotus parnellii Pteronotus parnelli Pteronotus parnelli Pteronotus parnelli Pteronotus parnelli Pteronotus parnelli Pteronotus parnellii Pteronotus parnelli Pteronotus parnellii Pteronotus parnelli Pteronotus parnelli Pteronotus parnellii Pteronotus parnellii Pteronotus parnelli Pteronotus parnelli Pteronotus parnelli Pteronotus parnelli Pteronotus parnelli Pteronotus parnelli Pteronotus parnellii Pteronotus parnelli Pteronotus parnellii
Guyan

Guyana

Guyana

Guyana

Guyana

Guyana

Guyana

Guyana

Guyana

Guyana

Guyana

Guyana

Guyana

Guyana

Guyana

Guyana

Guyana

Guyana

Guyana

Guyana

Guyana

Guyana

Guyana

Guyana

Guyana

Guyana

Guyana

Guyana

Guyana

Guyana

Guyana

Guyana

Guyana

Guyana

Guyana

Guyana

Guyana

Guyana

Guyana

Guyana

Guyana

Guyana

Guyana

Guyana

Guyana

Guyana

Guyana

Guyana

Guyana

Guyana

Guyana

Guyana

Guyana

Guyana

Guyana

Guyana

Guyana

Guyana

Guyana

Guyana

Guyana

Guyana

Guyana

Guyana

Guyana

Guyana

Guyana

Guyana

Guyana

Guyana

Guyana

Guyana

Guyana

Guyana

Guyana

Guyana

Guyana

Guyana

Guyana
Potaro-Siparuni Mount Daniel Cutlin 4.45 Potaro-Siparuni I Iwokrama Field Stat 4.667 Potaro-Siparuni Iwokrama Field Stat 4.667 Potaro-Siparuni I Iwokrama Field Stat 4.667 Potaro-Siparuni I Iwokrama Field Stat 4.667 Potaro-Siparuni Iwokrama Field Stat4.667 Potaro-Siparuni I Iwokrama Field Stat 4.667 Potaro-Siparuni I Iwokrama Field Stat 4.667 Demerara-Mahaic Madewini Creek Bri 6.5

Demerara-Mahaic Ceiba Biological St 6.5 Demerara-Mahaic Ceiba Biological St 6.5 Demerara-Mahaic Ceiba Biological Ste 6.5 Demerara-Mahaic Ceiba Biological St 6.5

Potaro-Siparuni Mount Ayanganna, 5.333 Potaro-Siparuni Potaro River, Malek 5.267 Potaro-Siparuni Mount Wokomung, $\mid 5.133$ Upper Demerara-I West Pibiri, Mabura 5.033 Upper Demerara-I West Pibiri, Mabura 5.033 Upper Demerara-I West Pibiri, Mabura 5.033 Upper Demerara-I West Pibiri, Mabura 5.033 Jper Demerara-I West Pibiri, Mabura 5.033 Upper Demerara-I West Pibiri, Mabura 5.033 Upper Demerara-I West Pibiri, Mabura 5.033 Upper Demerara-I West Pibiri, Mabura 5.033 Upper Demerara-I West Pibiri, Mabura 5.033 Upper Demerara-I West Pibiri, Mabura 5.033 Upper Demerara-I West Pibiri, Mabura 5.033 Upper Takutu-Upr Pobawau Creek Mo 3.267 Upper Takutu-Upr Cacique Mountain, I 3.183 Upper Takutu-Upr Cacique Mountain, I 3.183 Upper Takutu-Upr Cacique Mountain, I3.183 Upper Takutu-Upr Cacique Mountain, I3.183 Upper Takutu-Upr Cacique Mountain, I3.183 Upper Takutu-Upr Cacique Mountain, I 3.183 Upper Takutu-Upr Cacique Mountain, I3.183 Uper Takutu-Upr Cacique Mountain, I3.183 Upper Takutu-Upr Cacique Mountain, I 3.183 Upper Takutu-Upr Cacique Mountain, I 3.183 Upper Takutu-Upr Cacique Mountain, I3.183 Upper Takutu-Upr Cacique Mountain, | 3.183 Demerara-Mahaic Ceiba Biological Ce 6.5 Demerara-Mahaic Ceiba Biological Ce 6.5

Demerara-Mahaic Madewini Creek Bri 6.5 Demerara-Mahaic Ceiba Biological Ce 6.5

Demerara-Mahaic Ceiba Biological Ce 6.5 Demerara-Mahaic Ceiba Biological Ce 6.5

Essequibo Islands Shanklands 6.483

Essequibo Islands Shanklands $\quad 6.483 \quad-58.583$

Essequibo Islands Shanklands $\quad 6.483 \quad-58.583$ Essequibo Islands Shanklands $\quad 6.483 \quad-58.583$ Essequibo Islands Shanklands $\quad 6.483 \quad-58.583$ Essequibo Islands Shanklands $\quad 6.483 \quad-58.583$ Essequibo Islands Shanklands $\quad 6.483 \quad-58.583$ Essequibo Islands Shanklands $\quad 6.483 \quad-58.583$ Essequibo Islands Shanklands $\quad 6.483 \quad-58.583$ Essequibo Islands Shanklands $\quad 6.483 \quad-58.583$ Essequibo Islands Shanklands $\quad 6.483 \quad-58.583$ Essequibo Islands Shanklands $\quad 6.483 \quad-58.583$ Essequibo Islands Shanklands $\quad 6.483 \quad-58.583$ Essequibo Islands Shanklands $\quad 6.483 \quad-58.583$ Essequibo Islands Shanklands $\quad 6.483 \quad-58.583$ Essequibo Islands Shanklands $\quad 6.483 \quad-58.583$ Essequibo Islands Shanklands $\quad 6.483 \quad-58.583$ Essequibo Islands Shanklands $\quad 6.483 \quad-58.583$ Essequibo Islands Shanklands $\quad 6.483 \quad-58.583$ Essequibo Islands Shanklands $\quad 6.483 \quad-58.583$ Potaro-Siparuni I Iwokrama Field Stat $4.683 \quad-58.683$ Potaro-Siparuni Iwokrama Field Stat $4.683 \quad-58.683$ Potaro-Siparuni Iwokrama Field Stat $4.683 \quad-58.683$ Potaro-Siparuni Iwokrama Field Stat $4.683 \quad-58.683$ Potaro-Siparuni I I wokrama Field Stat $4.683 \quad-58.683$ Potaro-Siparuni Iwokrama Field Stat $4.683 \quad-58.683$ Potaro-Siparuni Iwokrama Field Stat $4.683 \quad-58.683$ Potaro-Siparuni Iwokrama Field Stat $4.683 \quad-58.683$ Potaro-Siparuni Iwokrama Field Stat $4.683 \quad-58.683$ Potaro-Siparuni Iwokrama Field Stat $4.683 \quad-58.683$ Potaro-Siparuni I Iwokrama Field Stat $4.683 \quad-58.683$ Potaro-Siparuni I Iwokrama Field Stat $4.683 \quad-58.683$ Potaro-Siparuni I Iwokrama Field Stat 4.683 $\quad-58.683$ Potaro-Siparuni Iwokrama Field Stat $4.683 \quad-58.683$ 


\begin{tabular}{|c|c|c|c|c|c|c|}
\hline F51064 & JF448176 & Pteronotus parnellii & Guyana & Potaro-Siparuni & Iwokrama Field Stat 4.683 & -58.683 \\
\hline F51065 & JF448177 & Pteronotus parnellii & Guyana & Potaro-Siparuni & Iwokrama Field Stat 4.683 & -58.683 \\
\hline F51098 & JF448178 & Pteronotus parnellii & Guyana & Potaro-Siparuni & Iwokrama Field Stat 4.683 & -58.683 \\
\hline F51099 & JF448179 & Pteronotus parnellii & Guyana & Potaro-Siparuni & Iwokrama Field Stat 4.683 & -58.683 \\
\hline F51104 & JF448182 & Pteronotus parnellii & Guyana & Potaro-Siparuni & Kaieteur National P: 5.166 & -59.493 \\
\hline F51120 & JF448183 & Pteronotus parnellii & Guyana & Potaro-Siparuni & Kaieteur National P: 5.175 & -59.481 \\
\hline F51121 & JF448184 & Pteronotus parnellii & Guyana & Potaro-Siparuni & Kaieteur National P: 5.175 & -59.481 \\
\hline F51122 & JF448185 & Pteronotus parnellii & Guyana & Potaro-Siparuni & Kaieteur National P: 5.175 & -59.481 \\
\hline F51137 & JF448186 & Pteronotus parnellii & Guyana & Potaro-Siparuni & Kaieteur National P: 5.175 & -59.481 \\
\hline F51162 & JF448187 & Pteronotus parnellii & Guyana & Potaro-Siparuni & Kaieteur National P: 5.175 & -59.481 \\
\hline F51163 & JF448188 & Pteronotus parnellii & Guyana & Potaro-Siparuni & Kaieteur National P: 5.175 & -59.481 \\
\hline F51164 & JF448189 & Pteronotus parnellii & Guyana & Potaro-Siparuni & Kaieteur National P: 5.175 & -59.481 \\
\hline F51195 & JF448190 & Pteronotus parnellii & Guyana & Potaro-Siparuni & Kaieteur National P: 5.175 & -59.481 \\
\hline F51198 & JF448191 & Pteronotus parnellii & Guyana & Potaro-Siparuni & Kaieteur National P: 5.175 & -59.481 \\
\hline F51206 & JF448192 & Pteronotus parnellii & Guyana & Potaro-Siparuni & Kaieteur National P: 5.166 & -59.493 \\
\hline F51207 & JF448193 & Pteronotus parnellii & Guyana & Potaro-Siparuni & Kaieteur National P: 5.166 & -59.493 \\
\hline F51208 & JF448194 & Pteronotus parnellii & Guyana & Potaro-Siparuni & Kaieteur National P: 5.166 & -59.493 \\
\hline F51209 & JF448195 & Pteronotus parnellii & Guyana & Potaro-Siparuni & Kaieteur National P: 5.166 & -59.493 \\
\hline F51211 & JF448196 & Pteronotus parnellii & Guyana & Potaro-Siparuni & Kaieteur National P: 5.175 & -59.481 \\
\hline F51246 & JF448166 & Pteronotus parnellii & Guyana & Potaro-Siparuni & Kaieteur National P: 5.166 & -59.493 \\
\hline F51249 & JF448167 & Pteronotus parnellii & Guyana & Potaro-Siparuni & Kaieteur National P: 5.175 & -59.481 \\
\hline F51263 & JF448168 & Pteronotus parnellii & Guyana & Potaro-Siparuni & Kaieteur National P: 5.166 & -59.493 \\
\hline F51264 & JF448169 & Pteronotus parnellii & Guyana & Potaro-Siparuni & Kaieteur National P:5.166 & -59.493 \\
\hline F51266 & JF448170 & Pteronotus parnellii & Guyana & Potaro-Siparuni & Kaieteur National P: 5.166 & -59.493 \\
\hline F51372 & JF455414 & Pteronotus parnellii & Guyana & Upper Takutu-Upr & Bbc Basecamp, Upı 3.506 & -58.233 \\
\hline F51383 & JF455412 & Pteronotus parnellii & Guyana & Upper Takutu-Upr & Bbc Basecamp, Up 3.506 & -58.233 \\
\hline F51395 & JF455417 & Pteronotus parnellii & Guyana & Upper Takutu-Upr & Bbc Basecamp, Up 3.506 & -58.233 \\
\hline F51418 & JF455418 & Pteronotus parnellii & Guyana & Upper Takutu-Upr & Bbc Basecamp, Up 3.506 & -58.233 \\
\hline F51433 & JF455416 & Pteronotus parnellii & Guyana & Upper Takutu-Upr & Bbc Basecamp, Upı 3.506 & -58.233 \\
\hline F51633 & JF455415 & Pteronotus parnellii & Guyana & Upper Takutu-Upr & $2 \mathrm{Km}$ W Of Unabaitı 2.279 & -59.435 \\
\hline F51675 & JF455413 & Pteronotus parnellii & Guyana & Upper Takutu-Upr & $2 \mathrm{Km}$ W Of Unabaitı 2.279 & -59.435 \\
\hline F52644 & JF459255 & Pteronotus parnellii & Guyana & Potaro-Siparuni & Iwokrama Forest, C 4.25 & -58.909 \\
\hline F52653 & JF459254 & Pteronotus parnellii & Guyana & Potaro-Siparuni & Iwokrama Forest, C 4.25 & -58.909 \\
\hline F52720 & JF459253 & Pteronotus parnellii & Guyana & Potaro-Siparuni & Iwokrama Forest, C 4.25 & -58.909 \\
\hline F52726 & JF459252 & Pteronotus parnellii & Guyana & Potaro-Siparuni & Iwokrama Forest, C 4.25 & -58.909 \\
\hline F52727 & JF459251 & Pteronotus parnellii & Guyana & Potaro-Siparuni & Iwokrama Forest, C 4.25 & -58.909 \\
\hline F52739 & JF459250 & Pteronotus parnellii & Guyana & Potaro-Siparuni & Iwokrama Forest, C 4.25 & -58.909 \\
\hline F52756 & JF459249 & Pteronotus parnellii & Guyana & Potaro-Siparuni & Iwokrama Forest, C 4.25 & -58.909 \\
\hline F52772 & JF459248 & Pteronotus parnellii & Guyana & Potaro-Siparuni & Iwokrama Forest, C 4.25 & -58.909 \\
\hline F52777 & JF459247 & Pteronotus parnellii & Guyana & Potaro-Siparuni & Iwokrama Forest, C 4.25 & -58.909 \\
\hline FN31112 & JF448214 & Pteronotus parnellii & Guyana & Upper Takutu-Upr & Quarter Mile Landin 3.917 & -59.1 \\
\hline FN31113 & JF448215 & Pteronotus parnellii & Guyana & Upper Takutu-Upr & Quarter Mile Landin 3.917 & -59.1 \\
\hline FN31129 & JF448216 & Pteronotus parnellii & Guyana & Upper Demerara-I & Kurupukari, East Ba 4.667 & -58.683 \\
\hline FN31130 & JF448217 & Pteronotus parnellii & Guyana & Upper Demerara-I & I Kurupukari, East Ba 4.667 & -58.683 \\
\hline FN33430 & & ABGYA093-C Pteronotus parnellii & Guyana & Upper Takutu-Upr & Karanambo $\quad 3.75$ & -59.3 \\
\hline FN33458 & JF448258 & Pteronotus parnellii & Guyana & Upper Takutu-Upr & Karanambo & -59.3 \\
\hline FN33464 & JF448259 & Pteronotus parnellii & Guyana & Upper Takutu-Upr & Annai & -59.133 \\
\hline FN33465 & JF448260 & Pteronotus parnellii & Guyana & Upper Takutu-Upr & Annai & -59.133 \\
\hline FN33466 & JF448211 & Pteronotus parnellii & Guyana & Upper Takutu-Upr & Annai & -59.133 \\
\hline FN33467 & JF448212 & Pteronotus parnellii & Guyana & Upper Takutu-Upr & Annai & -59.133 \\
\hline FN33468 & JF448213 & Pteronotus parnellii & Guyana & Upper Takutu-Upr & Annai & -59.133 \\
\hline F53472 & KT023253 & Pteronotus parnellii & JAMAICA & SAINT CATHERIN & ST. CLAIR CAVE, $\subseteq 18.15101$ & -77.0909 \\
\hline F53474 & KT023254 & Pteronotus parnellii & JAMAICA & SAINT CATHERIN & ST. CLAIR CAVE, $\subseteq 18.15101$ & -77.0909 \\
\hline F53489 & KT023255 & Pteronotus parnellii & JAMAICA & SAINT ELIZABET & 4 KM NE OF BALA( 18.20687 & -77.6258 \\
\hline F53491 & KT023256 & Pteronotus parnellii & JAMAICA & SAINT ELIZABET & 4 KM NE OF BALA( 18.20687 & -77.6258 \\
\hline F53527 & KT023257 & Pteronotus parnellii & JAMAICA & SAINT ANDREW & 10 KM N OF KINGS 18.08953 & -76.7237 \\
\hline F53528 & KT023258 & Pteronotus parnellii & JAMAICA & SAINT ANDREW & 10 KM N OF KINGS 18.08953 & -76.7237 \\
\hline F53531 & KT023259 & Pteronotus parnellii & JAMAICA & SAINT CATHERII & ST. CLAIR CAVE, $\leqslant 18.15101$ & -77.0909 \\
\hline F53532 & KT023260 & Pteronotus parnellii & JAMAICA & SAINT CATHERII & ST. CLAIR CAVE $\subseteq 18.15101$ & -77.0909 \\
\hline AVB0803: & HM208670 & Pteronotus parnellii & Mexico & Campeche & Vicinity of Zoh-Lagu 18.5836 & -89.4225 \\
\hline AVB0803: & HM208671 & Pteronotus parnellii & Mexico & Campeche & Vicinity of Zoh-Lagu 18.5836 & -89.4225 \\
\hline AVB0803: & HM208672 & Pteronotus parnellii & Mexico & Campeche & Vicinity of Zoh-Lagu 18.5971 & -89.4222 \\
\hline AVB0803: & HM208673 & Pteronotus parnellii & Mexico & Campeche & Vicinity of Zoh-Lagu 18.5971 & -89.4222 \\
\hline AVB0803: & HM208674 & Pteronotus parnellii & Mexico & Campeche & Vicinity of Zoh-Lagu 18.5971 & -89.4222 \\
\hline FN29532 & JF448279 & Pteronotus parnellii & Mexico & Campeche & $44 \mathrm{Km} \mathrm{S}$ Of Constitı 18.25 & -90.0667 \\
\hline F38035 & JF448119 & Pteronotus parnellii & Panama & Canal Zone & Gamboa $\quad 9.1$ & -79.7 \\
\hline F38044 & JF448337 & Pteronotus parnellii & Panama & Canal Zone & Gamboa $\quad 9.1$ & -79.7 \\
\hline F38062 & JF448264 & Pteronotus parnellii & Panama & Canal Zone & Parque Nacional Sc 9.15 & -79.75 \\
\hline F38103 & JF448263 & Pteronotus parnellii & Panama & & Parque Nacional Alt 8.683 & -79.933 \\
\hline F38104 & JF448338 & Pteronotus parnellii & Panama & & Parque Nacional Alt 8.683 & -79.933 \\
\hline F38105 & JF448339 & Pteronotus parnellii & Panama & & Parque Nacional Alt 8.683 & -79.933 \\
\hline F38154 & JF448262 & Pteronotus parnellii & Panama & Chiriqui & Ojo De Agua, 2 Km 8.867 & -82.75 \\
\hline
\end{tabular}




\begin{tabular}{|c|c|c|c|c|c|c|}
\hline F38155 & JF448340 & Pteronotus parnellii & Panama & Chiriqui & Ojo De Agua, 2 Km 8.867 & -82.75 \\
\hline F38180 & JF448261 & Pteronotus parnellii & Panama & Chiriqui & Santa Clara $\quad 8.833$ & -82.75 \\
\hline F38214 & JF448341 & Pteronotus parnellii & Panama & Darien & Estacion Pirre, Parc 8 & -77.717 \\
\hline F38228 & JF448342 & Pteronotus parnellii & Panama & Darien & Estacion Pirre, Parc 8 & -77.717 \\
\hline F41053 & JF448284 & Pteronotus parnellii & Suriname & Brokopondo & Brownsberg Nature 4.933 & -55.2 \\
\hline F41073 & JF448285 & Pteronotus parnellii & Suriname & Brokopondo & Brownsberg Nature 4.95 & -55.183 \\
\hline F41074 & & ABSRA055-0 Pteronotus parnellii & Suriname & Brokopondo & Brownsberg Nature 4.95 & -55.183 \\
\hline F41075 & & ABSRA056-0 Pteronotus parnellii & Suriname & Brokopondo & Brownsberg Nature 4.95 & -55.183 \\
\hline F41120 & JF448398 & Pteronotus parnellii & Suriname & Brokopondo & Brownsberg Nature 4.933 & -55.2 \\
\hline F41135 & JF448280 & Pteronotus parnellii & Suriname & Brokopondo & Brownsberg Nature 4.95 & -55.183 \\
\hline F41136 & & ABSRA097-0 Pteronotus parnellii & Suriname & Brokopondo & Brownsberg Nature 4.95 & -55.183 \\
\hline F41175 & & ABSRA126-0 Pteronotus parnellii & Suriname & Brokopondo & Brownsberg Nature 4.933 & -55.2 \\
\hline F41176 & & ABSRA127-0 Pteronotus parnellii & Suriname & Brokopondo & Brownsberg Nature 4.933 & -55.2 \\
\hline F41187 & & ABSRA136-0 Pteronotus parnellii & Suriname & Brokopondo & Brownsberg Nature 4.933 & -55.2 \\
\hline F41226 & JF448399 & Pteronotus parnellii & Suriname & Brokopondo & Brownsberg Nature 4.95 & -55.183 \\
\hline F41227 & & ABSRA157-0 Pteronotus parnellii & Suriname & Brokopondo & Brownsberg Nature 4.95 & -55.183 \\
\hline F41233 & & ABSRA163-0 Pteronotus parnellii & Suriname & Brokopondo & Brownsberg Nature 4.933 & -55.167 \\
\hline F41234 & & ABSRA164-0 Pteronotus parnellii & Suriname & Brokopondo & Brownsberg Nature 4.933 & -55.167 \\
\hline F41236 & & ABSRA166-0 Pteronotus parnellii & Suriname & Brokopondo & Brownsberg Nature 4.933 & -55.167 \\
\hline F41377 & & ABSRA249-0 Pteronotus parnellii & Suriname & Brokopondo & Brownsberg Nature 4.95 & -55.183 \\
\hline F41378 & & ABSRA250-0 Pteronotus parnellii & Suriname & Brokopondo & Brownsberg Nature 4.95 & -55.183 \\
\hline F52382 & & ABGYH044-1 Pteronotus parnellii & Suriname & Sipaliwini & Tafelberg, Base Caı 3.848 & -56.19 \\
\hline F52383 & & ABGYH045-1 Pteronotus parnellii & Suriname & Sipaliwini & Tafelberg, Base Caı 3.848 & -56.19 \\
\hline F52384 & JQ601315 & Pteronotus parnellii & Suriname & Sipaliwini & Tafelberg, Base Caı 3.848 & -56.19 \\
\hline F52385 & & ABGYH046-1 Pteronotus parnellii & Suriname & Sipaliwini & Tafelberg, Base Caı 3.848 & -56.19 \\
\hline F52391 & JQ601320 & Pteronotus parnellii & Suriname & Sipaliwini & Tafelberg, Base Caı 3.848 & -56.19 \\
\hline F52392 & JQ601321 & Pteronotus parnellii & Suriname & Sipaliwini & Tafelberg, Base Caı 3.848 & -56.19 \\
\hline F52399 & JQ601326 & Pteronotus parnellii & Suriname & Sipaliwini & Tafelberg, Base Caı 3.848 & -56.19 \\
\hline F52401 & JQ601328 & Pteronotus parnellii & Suriname & Sipaliwini & Tafelberg, Base Caı 3.848 & -56.19 \\
\hline F52402 & JQ601329 & Pteronotus parnellii & Suriname & Sipaliwini & Tafelberg, Base Caı 3.848 & -56.19 \\
\hline F52411 & JQ601337 & Pteronotus parnellii & Suriname & Sipaliwini & Tafelberg, Base Caı 3.848 & -56.19 \\
\hline F52414 & & ABSRA564-0 Pteronotus parnellii & Suriname & Sipaliwini & Tafelberg, Base Caı 3.848 & -56.19 \\
\hline F52421 & JQ601346 & Pteronotus parnellii & Suriname & Sipaliwini & Tafelberg, Base Caı 3.848 & -56.19 \\
\hline F52425 & JQ601349 & Pteronotus parnellii & Suriname & Sipaliwini & Tafelberg, Base Caı 3.848 & -56.19 \\
\hline F52543 & & ABGYH086-1 Pteronotus parnellii & Suriname & Sipaliwini & Tafelberg, Caiman ( 3.899 & -56.181 \\
\hline F52556 & JQ601402 & Pteronotus parnellii & Suriname & Sipaliwini & Tafelberg, Caiman ( 3.899 & -56.181 \\
\hline F52566 & JQ601408 & Pteronotus parnellii & Suriname & Sipaliwini & Tafelberg, Caiman ( 3.899 & -56.181 \\
\hline F52575 & & ABSRA725-0 Pteronotus parnellii & Suriname & Sipaliwini & Tafelberg, Caiman ( 3.899 & -56.181 \\
\hline F52576 & & ABGYH104-1 Pteronotus parnellii & Suriname & Sipaliwini & Tafelberg, Caiman ( 3.899 & -56.181 \\
\hline F52577 & JQ601410 & Pteronotus parnellii & Suriname & Sipaliwini & Tafelberg, Caiman ( 3.899 & -56.181 \\
\hline F52578 & JQ601411 & Pteronotus parnellii & Suriname & Sipaliwini & Tafelberg, Caiman ( 3.899 & -56.181 \\
\hline F52579 & JQ601412 & Pteronotus parnellii & Suriname & Sipaliwini & Tafelberg, Caiman ( 3.899 & -56.181 \\
\hline F52587 & JQ601417 & Pteronotus parnellii & Suriname & Sipaliwini & Tafelberg, Caiman ( 3.899 & -56.181 \\
\hline F52588 & JQ601418 & Pteronotus parnellii & Suriname & Sipaliwini & Tafelberg, Caiman ( 3.899 & -56.181 \\
\hline F52589 & JQ601419 & Pteronotus parnellii & Suriname & Sipaliwini & Tafelberg, Caiman ( 3.899 & -56.181 \\
\hline F52596 & JQ601422 & Pteronotus parnellii & Suriname & Sipaliwini & Tafelberg, Caiman ( 3.899 & -56.181 \\
\hline F52601 & JQ601423 & Pteronotus parnellii & Suriname & Sipaliwini & Tafelberg, Caiman ( 3.899 & -56.181 \\
\hline F52602 & JQ601424 & Pteronotus parnellii & Suriname & Sipaliwini & Tafelberg, Caiman ( 3.899 & -56.181 \\
\hline F53063 & HQ545477 & Pteronotus parnellii & Suriname & Sipaliwini & Iconja Landing, Sipc 1.994 & -56.092 \\
\hline F53082 & HQ919657 & Pteronotus parnellii & Suriname & Sipaliwini & Iconja Landing, Sipć 1.994 & -56.092 \\
\hline F53101 & HQ919674 & Pteronotus parnellii & Suriname & Sipaliwini & Iconja Landing, Sipć 1.994 & -56.092 \\
\hline F53127 & HQ919698 & Pteronotus parnellii & Suriname & Sipaliwini & Iconja Landing, Sipć 1.994 & -56.092 \\
\hline F53196 & HQ919758 & Pteronotus parnellii & Suriname & Sipaliwini & Sipaliwini Village $\quad 2.026$ & -56.124 \\
\hline F53268 & JQ601172 & Pteronotus parnellii & Suriname & Sipaliwini & Kutari River Camp 2.17526 & -56.788 \\
\hline F53299 & JQ601193 & Pteronotus parnellii & Suriname & Sipaliwini & Kutari River Camp 2.17526 & -56.788 \\
\hline F53312 & JQ601204 & Pteronotus parnellii & Suriname & Sipaliwini & Kutari River Camp 2.17526 & -56.788 \\
\hline F53322 & JQ601210 & Pteronotus parnellii & Suriname & Sipaliwini & Sipaliwini River Can 2.289 & -56.607 \\
\hline F53323 & JQ601211 & Pteronotus parnellii & Suriname & Sipaliwini & Sipaliwini River Can 2.289 & -56.607 \\
\hline F53324 & JQ601212 & Pteronotus parnellii & Suriname & Sipaliwini & Sipaliwini River Can 2.289 & -56.607 \\
\hline F53340 & JQ601222 & Pteronotus parnellii & Suriname & Sipaliwini & Sipaliwini River Can 2.289 & -56.607 \\
\hline F53341 & JQ601223 & Pteronotus parnellii & Suriname & Sipaliwini & Sipaliwini River Can 2.289 & -56.607 \\
\hline F53355 & JQ601237 & Pteronotus parnellii & Suriname & Sipaliwini & Sipaliwini River Can 2.289 & -56.607 \\
\hline F53373 & JQ601253 & Pteronotus parnellii & Suriname & Sipaliwini & Sipaliwini River Can 2.289 & -56.607 \\
\hline F53377 & JQ601258 & Pteronotus parnelliii & Suriname & Sipaliwini & Sipaliwini River Can 2.289 & -56.607 \\
\hline F53388 & JQ601267 & Pteronotus parnellii & Suriname & Sipaliwini & Sipaliwini River Can 2.289 & -56.607 \\
\hline F53394 & JQ601272 & Pteronotus parnellii & Suriname & Sipaliwini & Sipaliwini River Can 2.289 & -56.607 \\
\hline F53395 & JQ601273 & Pteronotus parnellii & Suriname & Sipaliwini & Sipaliwini River Can 2.289 & -56.607 \\
\hline F53404 & & ABSRA1380-Pteronotus parnellii & Suriname & Sipaliwini & Werehpai Camp 2.363 & -56.698 \\
\hline F53435 & & ABSRA1409-Pteronotus parnellii & Suriname & Sipaliwini & Werehpai Camp & -56.698 \\
\hline F53440 & & ABSRA1414- Pteronotus parnellii & Suriname & Sipaliwini & Werehpai Camp 2.363 & -56.698 \\
\hline F54222 & JF448331 & Pteronotus parnellii & Suriname & Sipaliwini & Bakhuis, Transect 94.478 & -57.042 \\
\hline F54283 & JF448332 & Pteronotus parnellii & Suriname & Sipaliwini & Bakhuis, Transect 94.478 & -57.042 \\
\hline F54302 & JF448333 & Pteronotus parnellii & Suriname & Sipaliwini & Bakhuis, Transect 94.478 & -57.042 \\
\hline F54306 & JF448334 & Pteronotus parnellii & Suriname & Sipaliwini & Bakhuis, Transect 94.478 & -57.042 \\
\hline F54375 & JF448335 & Pteronotus parnellii & Suriname & Sipaliwini & Bakhuis, Transect 84.476 & -57.057 \\
\hline F54391 & JF448336 & Pteronotus parnellii & Suriname & Sipaliwini & Bakhuis, Transect 94.478 & -57.042 \\
\hline F54497 & EU096921 & Pteronotus parnellii & Suriname & Sipaliwini & Bakhuis, Transect 14.661 & -57.178 \\
\hline F54508 & EU096911 & Pteronotus parnellii & Suriname & Sipaliwini & Bakhuis, Transect 14.661 & -57.178 \\
\hline F54519 & EU096925 & Pteronotus parnellii & Suriname & Sipaliwini & Bakhuis, Transect 14.661 & -57.178 \\
\hline F54534 & EU096902 & Pteronotus parnellii & Suriname & Sipaliwini & Bakhuis, Transect 14.661 & -57.178 \\
\hline
\end{tabular}




\begin{tabular}{|c|c|}
\hline 54535 & \\
\hline F54547 & \\
\hline F54548 & \\
\hline F54576 & EU096903 \\
\hline F54588 & EU096904 \\
\hline F54603 & EU096906 \\
\hline F54609 & EU096913 \\
\hline F54610 & EU096905 \\
\hline F54624 & EU096907 \\
\hline F54659 & EU096908 \\
\hline F54688 & EU096914 \\
\hline F54718 & EU096915 \\
\hline F54750 & EU096916 \\
\hline F54866 & EU096917 \\
\hline F54888 & EU096912 \\
\hline F54897 & EU09 \\
\hline F54912 & EUOS \\
\hline F54929 & EUOS \\
\hline F54975 & EUOS \\
\hline F55002 & EU096910 \\
\hline \multirow{2}{*}{\multicolumn{2}{|c|}{$\begin{array}{l}\text { Pparnellii- HQ932696 } \\
\text { Pparnellii- HQ932697 }\end{array}$}} \\
\hline & \\
\hline \multicolumn{2}{|c|}{ Pparnellii- HQ932698 } \\
\hline \multicolumn{2}{|c|}{ Pparnellii- HQ932699 } \\
\hline \multicolumn{2}{|c|}{ Pparnellii- HQ932700 } \\
\hline \multicolumn{2}{|c|}{ Pparnellii- HQ932701 } \\
\hline \multicolumn{2}{|c|}{ Pparnellii- HQ932702 } \\
\hline \multicolumn{2}{|c|}{ Pparnellii- HQ932703 } \\
\hline F42941 & JF448343 \\
\hline F42942 & JF4 \\
\hline F42950 & JF44 \\
\hline F42951 & JF44 \\
\hline F42952 & JF448281 \\
\hline F42953 & JF4 \\
\hline $\mathrm{F} 42$ & JF448 \\
\hline $\mathrm{F} 42$ & $\mathrm{JF} 4 \mathrm{C}$ \\
\hline $\mathrm{F} 42 \mathrm{~S}$ & JF448 \\
\hline F42959 & JF44 \\
\hline F43080 & \\
\hline F43101 & JF44 \\
\hline F43121 & JF448397 \\
\hline \multicolumn{2}{|c|}{ KF63682( KF636820 } \\
\hline FN31427 & JF446822 \\
\hline FN31428 & $\mathrm{JF} 4$ \\
\hline FN3142 & $\mathrm{JF} 4$ \\
\hline FN3143C & JF44 \\
\hline F38925 & EFO\& \\
\hline 39248 & JF45 \\
\hline F39349 & JF455425 \\
\hline F40844 & EF080597 \\
\hline F50412 & JF45 \\
\hline F50855 & EF080595 \\
\hline F50856 & JF455421 \\
\hline F50895 & JF455423 \\
\hline F50919 & JF455422 \\
\hline F50920 & JF455420 \\
\hline F50921 & JF455419 \\
\hline F50961 & EF080596 \\
\hline FN31128 & JF455429 \\
\hline FN33444 & JF455427 \\
\hline FN334 & JF455428 \\
\hline KC01168 & \\
\hline F54361 & JF44 \\
\hline 54369 & JF44 \\
\hline F54430 & JF447714 \\
\hline F54478 & JF44 \\
\hline F54518 & EU096949 \\
\hline F54558 & EU0969 \\
\hline F54566 & EU096935 \\
\hline F54567 & EU096948 \\
\hline F54568 & EU096937 \\
\hline F54589 & EU096939 \\
\hline F54612 & EU096934 \\
\hline F54613 & EU096927 \\
\hline F54631 & EU096929 \\
\hline F54632 & EU096930 \\
\hline F54633 & EU096932 \\
\hline F54634 & EU096926 \\
\hline F54658 & EU096950 \\
\hline F54680 & EU096941 \\
\hline
\end{tabular}

Pteronotus parnellii Pteronotus parnellii Pteronotus parnellii Pteronotus parnellii Pteronotus parnellii

Pteronotus parnellii Pteronotus parnellii Pteronotus parnellii Pteronotus parnellii Pteronotus parnellii Pteronotus parnellii Pteronotus parnellii Pteronotus parnellii Pteronotus parnellii Pteronotus parnellii Pteronotus parnellii Pteronotus parnellii Pteronotus parnellii Pteronotus parnellii Pteronotus parnellii

Pteronotus parnellii Pteronotus parnellii Pteronotus parnellii Pteronotus parnellii Pteronotus parnellii Pteronotus parnellii Pteronotus parnellii Pteronotus parnellii Pteronotus parnellii Pteronotus parnellii Pteronotus parnellii Pteronotus parnellii Pteronotus parnellii Pteronotus parnellii Pteronotus parnellii Pteronotus parnellii Pteronotus parnellii Pteronotus parnellii Pteronotus parnellii Pteronotus parnellii Pteronotus parnellii Pteronotus parnellii

Pteronotus personatus Pteronotus personatus Pteronotus personatus Pteronotus personatus Pteronotus personatus Pteronotus personatus Pteronotus personatus Pteronotus personatus Pteronotus personatus Pteronotus personatus Pteronotus personatus Pteronotus personatus Pteronotus personatus Pteronotus personatus Pteronotus personatus Pteronotus personatus Pteronotus personatus Pteronotus personatus Pteronotus personatus Pteronotus personatus Pteronotus personatus Pteronotus personatus Pteronotus personatus Pteronotus personatus Pteronotus personatus Pteronotus personatus Pteronotus personatus Pteronotus personatus Pteronotus personatus Pteronotus personatus Pteronotus personatus Pteronotus personatus Pteronotus personatus Pteronotus personatus Pteronotus personatus Pteronotus personatus Pteronotus personatus Pteronotus personatus

$\begin{array}{llll}\text { Suriname } & \text { Sipaliwini } & \text { Bakhuis, Transect 14.661 } & -57.178 \\ \text { Suriname } & \text { Sipaliwini } & \text { Bakhuis, Transect 14.661 } & -57.178 \\ \text { Suriname } & \text { Sipaliwini } & \text { Bakhuis, Transect 14.661 } & -57.178 \\ \text { Suriname } & \text { Sipaliwini } & \text { Bakhuis, Transect 14.661 } & -57.178 \\ \text { Suriname } & \text { Sipaliwini } & \text { Bakhuis, Transect 14.661 } & -57.178 \\ \text { Suriname } & \text { Sipaliwini } & \text { Bakhuis, Transect 14.661 } & -57.178 \\ \text { Suriname } & \text { Sipaliwini } & \text { Bakhuis, Transect 14.661 } & -57.178 \\ \text { Suriname } & \text { Sipaliwini } & \text { Bakhuis, Transect 14.661 } & -57.178 \\ \text { Suriname } & \text { Sipaliwini } & \text { Bakhuis, 1.8 km by 4.614 } & -57.161 \\ \text { Suriname } & \text { Sipaliwini } & \text { Bakhuis, Transect 14.714 } & -57.167 \\ \text { Suriname } & \text { Sipaliwini } & \text { Bakhuis, Transect 14.661 } & -57.178 \\ \text { Suriname } & \text { Sipaliwini } & \text { Bakhuis, Area 8 Cal4.542 } & -56.933 \\ \text { Suriname } & \text { Sipaliwini } & \text { Bakhuis, Area 8 Cal4.542 } & -56.933 \\ \text { Suriname } & \text { Sipaliwini } & \text { Bakhuis, Area 8 Reı 4.486 } & -56.867 \\ \text { Suriname } & \text { Sipaliwini } & \text { Blanche Marie Falls 4.811 } & -56.994 \\ \text { Suriname } & \text { Sipaliwini } & \text { Blanche Marie Falls 4.811 } & -56.994 \\ \text { Suriname } & \text { Sipaliwini } & \text { Blanche Marie Falls 4.811 } & -56.994 \\ \text { Suriname } & \text { Sipaliwini } & \text { Blanche Marie Falls 4.811 } & -56.994 \\ \text { Suriname } & \text { Sipaliwini } & \text { Blanche Marie Falls 4.811 } & -56.994 \\ \text { Suriname } & \text { Sipaliwini } & \text { Bakhuis Concessior 4.853 } & -56.781\end{array}$

Trinidad and Tobago

Trinidad and Tobago

Trinidad and Tobago

Trinidad and Tobago

Trinidad and Tobago

Trinidad and Tobago

Trinidad and Tobago

Trinidad and Tobago

Venezuela

Venezuela

Venezuela

Venezuela

Venezuela

Venezuela

Venezuela

Venezuela

Venezuela

Venezuela

Venezuela

Venezuela

Venezuela

Guatemala

Guatemala

Guatemala

Guatemala

Guyana

Guyana

Guyana

Guyana

Guyana

Guyana

Guyana

Guyana

Guyana

Guyana

Guyana

Guyana

Guyana

Guyana

Guyana

Mexico

Suriname

Suriname

Suriname

Suriname

Suriname

Suriname

Suriname

Suriname

Suriname

Suriname

Suriname

Suriname

Suriname

Suriname

Suriname

Suriname

Suriname

Suriname
Amazonas Pozon, $50 \mathrm{Km} \mathrm{Ne} \mathrm{C} 6.05$

Amazonas Pozon, $50 \mathrm{Km} \mathrm{Ne} \mathrm{C} 6.05$

Amazonas Pozon, $50 \mathrm{Km} \mathrm{Ne}$ C 6.05

Amazonas

Amazonas

Amazonas

Amazonas

Amazonas

Amazonas

Amazonas

Bolivar

Bolivar

Bolivar

Pozon, $50 \mathrm{Km} \mathrm{Ne} 06.05$

Pozon, $50 \mathrm{Km} \mathrm{Ne} \mathrm{C} 6.05$

Pozon, $50 \mathrm{Km} \mathrm{Ne} \mathrm{C} 6.05$

Pozon, $50 \mathrm{Km} \mathrm{Ne} \mathrm{C} 6.05$

Pozon, $50 \mathrm{Km} \mathrm{Ne} \mathrm{C} 6.05$

Pozon, $50 \mathrm{Km} \mathrm{Ne} \mathrm{C} 6.05$

Pozon, $50 \mathrm{Km} \mathrm{Ne} \mathrm{C6.05}$

Hato La Florida, 357.567

$3 \mathrm{Km}$ E Of Puerto C 7.167

$3 \mathrm{Km}$ E Of Puerto C 7.167

Alta Verapaz

Alta Verapaz

Grutas De Lanquin 15.5667 Grutas De Lanquin 15.567 Grutas De Lanquin 15.5667

Alta Verapaz

Iwokrama Reserve, 4.75

Potaro-Siparuni Iwokrama Reserve, 4.633

$\begin{array}{ll}\text { Potaro-Siparuni } & \text { Iwokrama Reserve, } 4.633 \\ \text { Potaro-Siparuni } & \text { Iwokrama Reserve, } 4.733\end{array}$

Potaro-Siparuni Pakatau Falls, Sipal 4.75

Upper Demerara-I West Pibiri, Mabura 5.033

Essequibo Islands Shanklands

Essequibo Islands Shanklands $\quad 6.483 \quad-58.583$

Essequibo Islands Shanklands $\quad 6.483 \quad-58.583$

Essequibo Islands Shanklands $\quad 6.483 \quad-58.583$

Essequibo Islands Shanklands $\quad 6.483 \quad-58.583$

Essequibo Islands Shanklands $\quad 6.483 \quad-58.583$

Essequibo Islands Shanklands $\quad 6.483 \quad-58.583$

Upper Takutu-Upr Karanambo $\quad 3.75 \quad-59.3$

Upper Takutu-Upr Karanambo $\quad 3.75 \quad-59.3$

Oaxaca, Tehuant

Sipaliwini

Sipaliwin

Sipaliwini

Sipaliwini

Sipaliwini

Sipaliwini

Sipaliwini

Sipaliwini

Sipaliwini

Sipaliwini

Sipaliwini

Sipaliwini

Sipaliwini

Sipaliwini

Sipaliwini

Sipaliwini

Sipaliwini

Sipaliwini
Bakhuis, Transect 94.478 Bakhuis, Transect 94.478 Bakhuis, Transect 74.529 Bakhuis, Transect 74.529 Bakhuis, Transect 14.661 Bakhuis, Transect 14.661 Bakhuis, Transect 14.661 Bakhuis, Transect 14.661 Bakhuis, Transect 14.661 Bakhuis, Transect 14.661 Bakhuis, $1.8 \mathrm{~km}$ by 4.614 Bakhuis, $1.8 \mathrm{~km}$ by 4.614 Bakhuis, $1.8 \mathrm{~km}$ by 4.614 Bakhuis, $1.8 \mathrm{~km}$ by 4.614 Bakhuis, $1.8 \mathrm{~km}$ by 4.614 Bakhuis, $1.8 \mathrm{~km}$ by 4.614 Bakhuis, Transect 14.714 Bakhuis, $1.8 \mathrm{~km}$ by 4.614
$-57.042$

$-57.042$

$-56.904$

$-56.904$

$-57.178$

$-57.178$

$-57.178$

$-57.178$

$-57.178$

$-57.178$

$-57.161$

$-57.161$

$-57.161$

$-57.161$

$-57.161$

$-57.161$

$-57.167$

$-57.161$
Grutas De Lanquin 15.5667

Upper Demerara-I Kurupukari, East Ba $4.667 \quad-58.683$ 


\begin{tabular}{|c|c|c|c|c|c|c|}
\hline F54681 & EU096942 & Pteronotus personatus & Suriname & Sipaliwini & Bakhuis, $1.8 \mathrm{~km}$ by 4.614 & -57.161 \\
\hline F54682 & EU096940 & Pteronotus personatus & Suriname & Sipaliwini & Bakhuis, $1.8 \mathrm{~km}$ by 4.614 & -57.161 \\
\hline F54687 & EU096946 & Pteronotus personatus & Suriname & Sipaliwini & Bakhuis, Transect 14.661 & -57.178 \\
\hline F54730 & EU096928 & Pteronotus personatus & Suriname & Sipaliwini & Bakhuis, Area 8 Cal 4.542 & -56.933 \\
\hline F54773 & EU096943 & Pteronotus personatus & Suriname & Sipaliwini & Bakhuis, Area 8 Reı 4.486 & -56.867 \\
\hline F54779 & EU096944 & Pteronotus personatus & Suriname & Sipaliwini & Bakhuis, Area 8 Reı 4.486 & -56.867 \\
\hline F54810 & EU096931 & Pteronotus personatus & Suriname & Sipaliwini & Bakhuis, Area 8 Cal 4.542 & -56.933 \\
\hline F54811 & EU096933 & Pteronotus personatus & Suriname & Sipaliwini & Bakhuis, Area 8 Cal 4.542 & -56.933 \\
\hline F54860 & EU096945 & Pteronotus personatus & Suriname & Sipaliwini & Bakhuis, Area 8 Reı 4.486 & -56.867 \\
\hline F54919 & EU096947 & Pteronotus personatus & Suriname & Sipaliwini & Blanche Marie Falls 4.811 & -56.994 \\
\hline F54938 & EU096936 & Pteronotus personatus & Suriname & Sipaliwini & Blanche Marie Falls 4.811 & -56.994 \\
\hline KC01168। & KC011686 & Pteronotus quadridens & Cuba & Guantanamo Prov & & \\
\hline F58959 & KX355118 & Pteronotus quadridens & Dominican Republic & Pedernales & $9 \mathrm{~km}$ NE of Pederne 18.08317 & -71.6709 \\
\hline F59212 & KX355119 & Pteronotus quadridens & Dominican Republic & Sanchez Ramirez & Cueva Honda de Ju 19.13234 & -70.0796 \\
\hline F59213 & KX355120 & Pteronotus quadridens & Dominican Republic & Sanchez Ramirez & Cueva Honda de Ju 19.13234 & -70.0796 \\
\hline F59214 & $\mathrm{K} X 355121$ & Pteronotus quadridens & Dominican Republic & Sanchez Ramirez & Cueva Honda de Ju 19.13234 & -70.0796 \\
\hline F59263 & KX355122 & Pteronotus quadridens & Dominican Republic & La Altagracia & Laguna Bavaro $\quad 18.64782$ & -68.364 \\
\hline F59264 & KX355123 & Pteronotus quadridens & Dominican Republic & La Altagracia & Laguna Bavaro & -68.364 \\
\hline F59265 & KX355124 & Pteronotus quadridens & Dominican Republic & La Altagracia & Laguna Bavaro & -68.364 \\
\hline F59284 & KX355125 & Pteronotus quadridens & Dominican Republic & La Altagracia & Barcelo Bavaro Res 18.65971 & -68.3942 \\
\hline F59285 & KX355126 & Pteronotus quadridens & Dominican Republic & La Altagracia & Barcelo Bavaro Res 18.65277 & -68.4001 \\
\hline F59286 & KX355127 & Pteronotus quadridens & Dominican Republic & La Altagracia & Barcelo Bavaro Res 18.65277 & -68.4001 \\
\hline F59287 & KX355128 & Pteronotus quadridens & Dominican Republic & La Altagracia & Barcelo Bavaro Res 18.65277 & -68.4001 \\
\hline F59307 & KX355129 & Pteronotus quadridens & Dominican Republic & La Altagracia & Punta Cana Ecologi 18.52531 & -68.3808 \\
\hline F53468 & KT023244 & Pteronotus quadridens & JAMAICA & SAINT CATHERIN & ST. CLAIR CAVE, $\leqq 18.15101$ & -77.0909 \\
\hline F53469 & KT023245 & Pteronotus quadridens & JAMAICA & SAINT CATHERIN & ST. CLAIR CAVE, $\subseteq 18.15101$ & -77.0909 \\
\hline F53485 & KT023246 & Pteronotus quadridens & JAMAICA & SAINT ELIZABET & $-1 \mathrm{KM}$ NE OF BALAC 18.18281 & -77.6437 \\
\hline F53486 & KT023247 & Pteronotus quadridens & JAMAICA & SAINT ELIZABET & $1 \mathrm{KM}$ NE OF BALA( 18.18281 & -77.6437 \\
\hline F53493 & КT023248 & Pteronotus quadridens & JAMAICA & SAINT ELIZABET & 4 KM NE OF BALAC 18.20687 & -77.6258 \\
\hline F53537 & КT023249 & Pteronotus quadridens & JAMAICA & SAINT CATHERIN & ST. CLAIR CAVE, $\subseteq 18.15101$ & -77.0909 \\
\hline F53539 & КT023250 & Pteronotus quadridens & JAMAICA & SAINT CATHERIN & ST. CLAIR CAVE, $\leqq 18.15101$ & -77.0909 \\
\hline F53540 & КT023251 & Pteronotus quadridens & JAMAICA & SAINT CATHERIN & ST. CLAIR CAVE, $\subseteq 18.15101$ & -77.0909 \\
\hline F53541 & KT023252 & Pteronotus quadridens & JAMAICA & SAINT CATHERIN & ST. CLAIR CAVE, $\leqq 18.15101$ & -77.0909 \\
\hline F44351 & JF446389 & Sturnira lilium & Brazil & Sao Paulo & Caetetus Ecological -22.383 & -49.667 \\
\hline F44363 & EF546827 & Sturnira lilium & Brazil & Sao Paulo & Caetetus Ecological -22.383 & -49.667 \\
\hline F44364 & EF546826 & Sturnira lilium & Brazil & Sao Paulo & Caetetus Ecological -22.383 & -49.667 \\
\hline F44368 & JF446386 & Sturnira lilium & Brazil & Sao Paulo & Caetetus Ecological -22.383 & -49.667 \\
\hline F44379 & & Sturnira lilium & Brazil & Sao Paulo & Caetetus Ecological -22.383 & -49.667 \\
\hline $\mathrm{F} 44380$ & EF546825 & Sturnira lilium & Brazil & Sao Paulo & Caetetus Ecological -22.383 & -49.667 \\
\hline F44447 & EF546824 & Sturnira lilium & Brazil & Sao Paulo & Caetetus Ecological -22.383 & -49.667 \\
\hline F44456 & JF446387 & Sturnira lilium & Brazil & Sao Paulo & Caetetus Ecological -22.383 & -49.667 \\
\hline F44463 & JF446388 & Sturnira lilium & Brazil & Sao Paulo & Caetetus Ecological -22.383 & -49.667 \\
\hline$B C Q C A Z$ & -1659.1 & Sturnira lilium & Ecuador & Pichincha & Nanegal, comunidar 0.12 & -78.67 \\
\hline BCQCAZ. & JN659667 & Sturnira lilium & Ecuador & Esmeraldas & Terminal terrestre d 0.95 & -79.65 \\
\hline BCQCAZ. & JN659655 & Sturnira lilium & Ecuador & Pichincha & Union del Rio Toact -0.32 & -78.95 \\
\hline DTS-145 & JN659654 & Sturnira lilium & Ecuador & Sucumbios & Cuyabeno, R.P.F., l -0.25 & -75.87 \\
\hline DTS-281 & & Sturnira lilium & Ecuador & Napo & Yasuni, P.N. Pompe -0.63 & -76.57 \\
\hline DTS-297 & JN659665 & Sturnira lilium & Ecuador & Loja & San Pedro de Vilcal & \\
\hline DTS-301 & JN659662 & Sturnira lilium & Ecuador & Loja & San Pedro de Vilcal & \\
\hline DTS-302 & JN659669 & Sturnira lilium & Ecuador & Loja & San Pedro de Vilcal & \\
\hline DTS-303 & JN659661 & Sturnira lilium & Ecuador & Loja & San Pedro de Vilcal & \\
\hline DTS-308 & JN659660 & Sturnira lilium & Ecuador & Loja & San Pedro de Vilcal & \\
\hline DTS-312 & JN659659 & Sturnira lilium & Ecuador & Loja & San Pedro de Vilcal & \\
\hline DTS-545 & & Sturnira lilium & Ecuador & Zamora Chinchipe & $€$ Rio Nangaritza, She -4.35 & -78.67 \\
\hline EPN-974! & JN659679 & Sturnira lilium & Ecuador & Napo & Canton Loreto, San -0.83 & -77.48 \\
\hline F37069 & JF449165 & Sturnira lilium & Ecuador & Napo & Parque Nacional Ya -0.65 & -76.45 \\
\hline F37178 & JF449166 & Sturnira lilium & Ecuador & Napo & Parque Nacional Ya -0.617 & -76.467 \\
\hline F37179 & JF449167 & Sturnira lilium & Ecuador & Napo & Parque Nacional Ya -0.617 & -76.467 \\
\hline F37180 & JF449168 & Sturnira lilium & Ecuador & Napo & Parque Nacional Ya -0.617 & -76.467 \\
\hline F37226 & EF546833 & Sturnira lilium & Ecuador & Napo & Parque Nacional Ya -0.8 & -76.4 \\
\hline $\mathrm{F} 37250$ & EF546832 & Sturnira lilium & Ecuador & Napo & Parque Nacional Ya-0.8 & -76.4 \\
\hline F37350 & JF449170 & Sturnira lilium & Ecuador & Napo & Parque Nacional Ya -0.783 & -76.433 \\
\hline F37356 & JF449171 & Sturnira lilium & Ecuador & Napo & Parque Nacional Ya -0.833 & -76.333 \\
\hline F37357 & JF449172 & Sturnira lilium & Ecuador & Napo & Parque Nacional Ya -0.833 & -76.333 \\
\hline F37358 & JF449173 & Sturnira lilium & Ecuador & Napo & Parque Nacional Ya -0.833 & -76.333 \\
\hline F37453 & JF449174 & Sturnira lilium & Ecuador & Napo & Parque Nacional Ya -0.65 & -76.45 \\
\hline F37490 & JF449175 & Sturnira lilium & Ecuador & Napo & Parque Nacional Ya -0.633 & -76.45 \\
\hline F37565 & JF449176 & Sturnira lilium & Ecuador & Napo & Parque Nacional Ya -0.833 & -76.333 \\
\hline F37577 & JF449177 & Sturnira lilium & Ecuador & Napo & Parque Nacional Ya -0.833 & -76.333 \\
\hline F37578 & JF449178 & Sturnira lilium & Ecuador & Napo & Parque Nacional Ya -0.833 & -76.333 \\
\hline F37579 & JF449179 & Sturnira lilium & Ecuador & Napo & Parque Nacional Ya -0.833 & -76.333 \\
\hline
\end{tabular}


F37592 EF546831

F37799 JF449183

F37828 JF449169

F37940 EF546830

F37941 JF449184

F37954 EF546829

F37977 JF449185

F37978 JF449186

F40145 JF449188

F40201 JF449189

F40202 JF449190

F40203 JF449191

F40217 JF449192

F40344 JF449180

F40345

F40346 JF449181

F40348 JF449182

F40510 JF449158

F40511 JF449159

F40512 JF449160

F40518 JF449161

F40530 JF449162

F40531 JF449163

F40532 JF449164

MECN-13 JN659672

MECN-13 JN659650

MECN-13 JN659642

MECN-1394BC.1

MECN-13 JN659649

MECN-20 JN659676

MECN-61 JN659644

MECN-67 JN659666

MECN-67 JN659643

MECN-77 JN659648

MECN-86 JN659646

MECN-88 JN659641

MECN-97 JN659647

MECN-97 JN659645

NQP-8 JN659656

PJV-013 JN659668

PJV-014

PJV-015

PJV-016

PJV-017 JN659651

PJV-018 JN659684

PJV-035 JN659680

PJV-036

PJV-261 JN659652

PJV-395 JN659639

PJV-577 JN659673

PJV-579 JN659670

PJV-580 JN659663

PJV-583 JN659664

PJV-905 JN659640

QCAZ-10 JN659658

QCAZ-16 JN659685

QCAZ-42 JN659677

QCAZ-44 JN659671

QCAZ-47 JN659638

QCAZ-47. JN659690

QCAZ-93 JN659678

QLP-082 JN659675

RMFN-14 JN659692

RMFN-16 JN659691

RMFN-21 JN659689

SB-66 JN659683

SB-67 JN659682

SB-83 JN659688

SB-85 JN659681

SB-89 JN659687

SB-90 JN659674

SB-91 JN659686

SB-93 JN659653

SC-9529 JN659657

ST-017

F35401 EF546839

F35402 JF446545

F35403 JF446546

F35404 JF446547

F35415 JF446548
Sturnira lilium

Sturnira lilium

Sturnira lilium

Sturnira lilium

Sturnira lilium

Sturnira lilium

Sturnira lilium

Sturnira lilium

Sturnira lilium

Sturnira lilium

Sturnira lilium

Sturnira lilium

Sturnira lilium

Sturnira lilium

Sturnira lilium

Sturnira lilium

Sturnira lilium

Sturnira lilium

Sturnira lilium

Sturnira lilium

Sturnira lilium

Sturnira lilium

Sturnira lilium

Sturnira lilium

Sturnira lilium

Sturnira lilium

Sturnira lilium

Sturnira lilium

Sturnira lilium

Sturnira lilium

Sturnira lilium

Sturnira lilium

Sturnira lilium

Sturnira lilium

Sturnira lilium

Sturnira lilium

Sturnira lilium

Sturnira lilium

Sturnira lilium

Sturnira lilium

Sturnira lilium

Sturnira lilium

Sturnira lilium

Sturnira lilium

Sturnira lilium

Sturnira lilium

Sturnira lilium

Sturnira lilium

Sturnira lilium

Sturnira lilium

Sturnira lilium

Sturnira lilium

Sturnira lilium

Sturnira lilium

Sturnira lilium

Sturnira lilium

Sturnira lilium

Sturnira lilium

Sturnira lilium

Sturnira lilium

Sturnira lilium

Sturnira lilium

Sturnira lilium

Sturnira lilium

Sturnira lilium

Sturnira lilium

Sturnira lilium

Sturnira lilium

Sturnira lilium

Sturnira lilium

Sturnira lilium

Sturnira lilium

Sturnira lilium

Sturnira lilium

Sturnira lilium

Sturnira lilium

Sturnira lilium

Sturnira lilium

Sturnira lilium

Sturnira lilium

$\begin{array}{llll}\text { Ecuador } & \text { Napo } & \text { Parque Nacional Ya -0.8 } & -76.4 \\ \text { Ecuador } & \text { Napo } & \text { Parque Nacional Ya -0.833 } & -76.35 \\ \text { Ecuador } & \text { Napo } & \text { Parque Nacional Ya -0.833 } & -76.35 \\ \text { Ecuador } & \text { Napo } & \text { Parque Nacional Ya -0.8 } & -76.4 \\ \text { Ecuador } & \text { Napo } & \text { Parque Nacional Ya -0.8 } & -76.4 \\ \text { Ecuador } & \text { Napo } & \text { Parque Nacional Ya -0.8 } & -76.4 \\ \text { Ecuador } & \text { Napo } & \text { Parque Nacional Ya -0.833 } & -76.333 \\ \text { Ecuador } & \text { Napo } & \text { Parque Nacional Ya -0.833 } & -76.333 \\ \text { Ecuador } & \text { Napo } & \text { Parque Nacional Ya -0.65 } & -76.45 \\ \text { Ecuador } & \text { Napo } & \text { Parque Nacional Ya -0.8 } & -76.4 \\ \text { Ecuador } & \text { Napo } & \text { Parque Nacional Ya-0.8 } & -76.4 \\ \text { Ecuador } & \text { Napo } & \text { Parque Nacional Ya -0.8 } & -76.4 \\ \text { Ecuador } & \text { Napo } & \text { Parque Nacional Ya-0.8 } & -76.4\end{array}$

Ecuador

Ecuador

Ecuador

Ecuador

Ecuador

Ecuador

Ecuador

Ecuador

Ecuador

Ecuador

Ecuador

Ecuador

Ecuador

Ecuador

Ecuador

Ecuador

Ecuador

Ecuador

Ecuador

Ecuador

Ecuador

Ecuador

Ecuador

Ecuador

Ecuador

Ecuador

Ecuador

Ecuador

Ecuador

Ecuador

Ecuador

Ecuador

Ecuador

Ecuador

Ecuador

Ecuador

Ecuador

Ecuador

Ecuador

Ecuador

Ecuador

Ecuador

Ecuador

Ecuador

Ecuador

Ecuador

Ecuador

Ecuador

Ecuador

Ecuador

Ecuador

Ecuador

Ecuador

Ecuador

Ecuador

Ecuador

Ecuador

Ecuador

Ecuador

Ecuador

Ecuador

El Salvador

El Salvador

El Salvador

El Salvador

El Salvador

Parque Nacional Ya

Parque Nacional Ya

Parque Nacional Ya

Parque Nacional Ya

Parque Nacional Ya -0.833 $\quad-76.333$

Parque Nacional Ya $-0.833 \quad-76.333$

Parque Nacional Ya - $0.833 \quad-76.333$

Parque Nacional Ya -0.833 $\quad-76.333$

Parque Nacional Ya

$\begin{array}{ll}\text { Napo } & \text { Parque Nacional Ya } \\ \text { Napo } & \text { Parque Nacional Ya }\end{array}$

$\begin{array}{ll}\text { Napo } & \text { Parque Nacional Ya } \\ \text { Napo } & \text { Parque Nacional Ya }\end{array}$

Morona-Santiago P. N. Sangay, Coorı

Morona-Santiago P. N. Sangay, Coorı

Parque Nacional Sa

Parque Nacional Sa

Parque Nacional Sa

Sucumbios Tarapoa, Comunida

Napo P. N. Yasuni, $24 \mathrm{Km}$

Napo P. N. Yasuni, $59 \mathrm{Km}$

Napo P. N. Yasuni, el Sali

Napo P. N. Yasuni, El Sal

Napo P. N. Yasuni, $73 \mathrm{Km}$

P. N. Yasuni, $66 \mathrm{Km}$

Napo

Napo

Napo

Napo

Napo

Pichincha

Pichincha

Pichincha

Pichincha

Manabi

Napo

Pichincha

Pichincha

Pichincha

Pichincha

Loja

Pichincha

Pichincha

Pichincha

Loja

Pichincha

Pichincha

Pichincha

Pichincha

Orellana

Orellana

Pastaza

Pichincha

Pichincha

Pichincha

Pichincha

Pichincha

Pichincha

Pichincha

Pichincha

Azuay 
F35444 JF446549 F35479 EF546818

F35480 JF448134 F35481 JF446550 F35517 JF446551

F35518 EF546838

F34049 JF446857

F34050 JF446858

F34051 JF446859

F34052 JF446860

F34053 JF446861

F34146 JF446852

F34147 JF446853

F34148 EF546840

FN31385 JF446862

FN31386 JF446863

FN31387 JF446864

FN31388 JF446865

FN31389 JF446866

FN31461 JF446867

FN31462 JF446868

FN31463 JF446869

FN31464 JF446870

FN31773 JF446830

FN31816 JF446829

FN31817 JF446841

FN31818 JF446842

FN31835 JF446844

FN31836 JF446845

FN31837 JF446846

FN31838 JF446847

FN31839 JF446848

FN31886 JF446849

FN31887 JF446850

FN31888 JF446851

FN31889 JF446854

FN31941 JF446855

FN31982 JF446856

FN32285 JF446831

FN32286

FN32287 JF446832

FN32288 JF446833

FN32289 JF446834

FN32336 EF546844

FN32337 EF546843

FN32338 JF446835

FN32339 JF446836

FN32358 EF546842

FN32359 EF546841

FN32360 JF446837

FN32361 JF446838

FN32362 JF446839

FN32363 JF446840

F34999 JF455762

F35023 JF455763

F35119 EF080684

F35134 EF080685

F35135 JF455781

F35136 JF455782

F35147 JF455783

F35151 EF080686

F35152 EF080687

F35166 JF455784

F35167 EF080688

F35168 JF455785

F36355 JF455786

F36356 JF455787

F36390 JF455788

F36406 JF455789

F36473 JF455790

F36474 JF455791

F36475 JF455792

F36505 JF455793

F36540 JF455794

F36541 JF455795

F36542 JF455796

F36543 JF455797

F36544 JF455798

F36549 JF455799

F36553 JF455800
Sturnira lilium

Sturnira lilium

Sturnira lilium

Sturnira lilium

Sturnira lilium

Sturnira lilium

Sturnira lilium

Sturnira lilium

Sturnira lilium

Sturnira lilium

Sturnira lilium

Sturnira lilium

Sturnira lilium

Sturnira lilium

Sturnira lilium

Sturnira lilium

Sturnira lilium

Sturnira lilium

Sturnira lilium

Sturnira lilium

Sturnira lilium

Sturnira lilium

Sturnira lilium

Sturnira lilium

Sturnira lilium

Sturnira lilium

Sturnira lilium

Sturnira lilium

Sturnira lilium

Sturnira lilium

Sturnira lilium

Sturnira lilium

Sturnira lilium

Sturnira lilium

Sturnira lilium

Sturnira lilium

Sturnira lilium

Sturnira lilium

Sturnira lilium

Sturnira lilium

Sturnira lilium

Sturnira lilium

Sturnira lilium

Sturnira lilium

Sturnira lilium

Sturnira lilium

Sturnira lilium

Sturnira lilium

Sturnira lilium

Sturnira lilium

Sturnira lilium

Sturnira lilium

Sturnira lilium

Sturnira lilium

Sturnira lilium

Sturnira lilium

Sturnira lilium

Sturnira lilium

Sturnira lilium

Sturnira lilium

Sturnira lilium

Sturnira lilium

Sturnira lilium

Sturnira lilium

Sturnira lilium

Sturnira lilium

Sturnira lilium

Sturnira lilium

Sturnira lilium

Sturnira lilium

Sturnira lilium

Sturnira lilium

Sturnira lilium

Sturnira lilium

Sturnira lilium

Sturnira lilium

Sturnira lilium

Sturnira lilium

Sturnira lilium

Sturnira lilium

$\begin{array}{llll}\text { El Salvador } & \text { Ahuachapan } & \text { El Imposible, San F 13.85 } & -90 \\ \text { El Salvador } & \text { Ahuachapan } & \text { El Imposible, El Ref 13.8 } & -90 \\ \text { El Salvador } & \text { Ahuachapan } & \text { El Imposible, El Ref 13.8 } & -90 \\ \text { El Salvador } & \text { Ahuachapan } & \text { El Imposible, El Ref } 13.8 & -90 \\ \text { El Salvador } & \text { Ahuachapan } & \text { El Imposible, El Ref 13.8 } & -90 \\ \text { El Salvador } & \text { Ahuachapan } & \text { El Imposible, El Ref } 13.8 & -90\end{array}$

El Salvado

Guatemala

Guatemala

Guatemala

Guatemala

Guatemala

Guatemala

Guatemala

Guatemala

Guatemala

Guatemala

Guatemala

Guatemala

Guatemala

Guatemala

Guatemala

Guatemala

Guatemala

Guatemala

Guatemala

Guatemala

Guatemala

Guatemala

Guatemala

Guatemala

Guatemala

Guatemala

Guatemala

Guatemala

Guatemala

Guatemala

Guatemala

Guatemala

Guatemala

Guatemala

Guatemala

Guatemala

Guatemala

Guatemala

Guatemala

Guatemala

Guatemala

Guatemala

Guatemala

Guatemala

Guatemala

Guatemala

Guatemala

Guyana

Guyana

Guyana

Guyana

Guyana

Guyana

Guyana

Guyana

Guyana

Guyana

Guyana

Guyana

Guyana

Guyana

Guyana

Guyana

Guyana

Guyana

Guyana

Guyana

Guyana

Guyana

Guyana

Guyana

Guyana

Guyana

Guyana

Ahuachapan

El Progreso

El Progreso

El Progreso

EI Progreso

Solola

Solola

Solola

El Progreso

El Progreso

El Progreso

El Progreso

El Progreso

El Progreso

El Progreso

El Progreso

El Progreso

Peten

Peten

Peten

Peten

Peten

Peten

Peten

Peten

Peten

Peten

Peten

Peten

Peten

Peten

Peten

Peten

Peten

Peten

Peten

Peten

Peten

Peten

Peten

Peten

Peten

Peten

Peten

Peten

Peten

Peten

Barima-Waini

Barima-Waini

Barima-Waini

Barima-Wain

Barima-Waini

Barima-Waini

Barima-Waini 


\begin{tabular}{|c|c|c|c|c|c|c|}
\hline F36574 & JF455801 & Sturnira lilium & Guyana & \multicolumn{2}{|c|}{ Upper Takutu-Upr $5 \mathrm{Km}$ Se Of Surame 4.1} & -59.05 \\
\hline F36575 & JF455802 & Sturnira lilium & Guyana & \multicolumn{2}{|c|}{ Upper Takutu-Upr $5 \mathrm{Km}$ Se Of Surame 4.1} & -59.05 \\
\hline F36576 & JF455803 & Sturnira lilium & Guyana & \multicolumn{2}{|c|}{ Upper Takutu-Upr $5 \mathrm{Km}$ Se Of Surame 4.1} & -59.05 \\
\hline F36577 & JF455804 & Sturnira lilium & Guyana & \multicolumn{2}{|c|}{ Upper Takutu-Upr $5 \mathrm{Km}$ Se Of Surame 4.1} & -59.05 \\
\hline F36578 & JF455805 & Sturnira lilium & Guyana & \multicolumn{2}{|c|}{ Upper Takutu-Upr $5 \mathrm{Km}$ Se Of Surame 4.1} & -59.05 \\
\hline F36579 & JF455806 & Sturnira lilium & Guyana & \multicolumn{2}{|c|}{ Upper Takutu-Upr $5 \mathrm{Km}$ Se Of Surame 4.1} & -59.05 \\
\hline F36628 & JF455807 & Sturnira lilium & Guyana & Upper Takutu-Upr & $5 \mathrm{Km}$ Se Of Surame 4.1 & -59.05 \\
\hline F36629 & JF455808 & Sturnira lilium & Guyana & Upper Takutu-Upr & $5 \mathrm{Km}$ Se Of Surame 4.1 & -59.05 \\
\hline F36630 & JF455809 & Sturnira lilium & Guyana & Upper Takutu-Upr & $5 \mathrm{Km}$ Se Of Surame 4.1 & -59.05 \\
\hline F36631 & JF455810 & Sturnira lilium & Guyana & Upper Takutu-Upr & $5 \mathrm{Km}$ Se Of Surame 4.1 & -59.05 \\
\hline F36632 & JF455811 & Sturnira lilium & Guyana & Upper Takutu-Upr & $5 \mathrm{Km}$ Se Of Surame 4.1 & -59.05 \\
\hline F36633 & JF455812 & Sturnira lilium & Guyana & Upper Takutu-Upr & $5 \mathrm{Km}$ Se Of Surame 4.1 & -59.05 \\
\hline F36634 & JF455813 & Sturnira lilium & Guyana & Upper Takutu-Upr & $5 \mathrm{Km}$ Se Of Surame 4.1 & -59.05 \\
\hline F36635 & JF455814 & Sturnira lilium & Guyana & Upper Takutu-Upr & $5 \mathrm{Km}$ Se Of Surame 4.1 & -59.05 \\
\hline F36684 & JF455815 & Sturnira lilium & Guyana & Upper Takutu-Upr & $5 \mathrm{Km}$ Se Of Surame 4.1 & -59.05 \\
\hline F36685 & JF455816 & Sturnira lilium & Guyana & Upper Takutu-Upr & $5 \mathrm{Km}$ Se Of Surame 4.1 & -59.05 \\
\hline F36686 & JF455817 & Sturnira lilium & Guyana & Upper Takutu-Upp & $5 \mathrm{Km}$ Se Of Surame 4.1 & -59.05 \\
\hline F36728 & JF455764 & Sturnira lilium & Guyana & Upper Takutu-Upr & $5 \mathrm{Km}$ Se Of Surame 4.1 & -59.05 \\
\hline F36729 & JF455765 & Sturnira lilium & Guyana & Upper Takutu-Upr & $5 \mathrm{Km}$ Se Of Surame 4.1 & -59.05 \\
\hline F36730 & JF455766 & Sturnira lilium & Guyana & Upper Takutu-Upr & $5 \mathrm{Km}$ Se Of Surame 4.1 & -59.05 \\
\hline F36731 & JF455767 & Sturnira lilium & Guyana & Upper Takutu-Upr & $5 \mathrm{Km}$ Se Of Surame 4.1 & -59.05 \\
\hline F36732 & JF455768 & Sturnira lilium & Guyana & Upper Takutu-Upr & $5 \mathrm{Km}$ Se Of Surame 4.1 & -59.05 \\
\hline F36769 & JF455769 & Sturnira lilium & Guyana & Upper Takutu-Upr & $5 \mathrm{Km}$ Se Of Surame 4.1 & -59.05 \\
\hline F36770 & JF455770 & Sturnira lilium & Guyana & Upper Takutu-Upr & $5 \mathrm{Km}$ Se Of Surame 4.1 & -59.05 \\
\hline F36771 & JF455771 & Sturnira lilium & Guyana & Upper Takutu-Upr & $5 \mathrm{Km}$ Se Of Surame 4.1 & -59.05 \\
\hline F36772 & JF455772 & Sturnira lilium & Guyana & Upper Takutu-Upr & $5 \mathrm{Km}$ Se Of Surame 4.1 & -59.05 \\
\hline F36773 & JF455773 & Sturnira lilium & Guyana & Upper Takutu-Upr & $5 \mathrm{Km}$ Se Of Surame 4.1 & -59.05 \\
\hline F36774 & JF455774 & Sturnira lilium & Guyana & Upper Takutu-Upr & $5 \mathrm{Km}$ Se Of Surame 4.1 & -59.05 \\
\hline F36775 & JF455775 & Sturnira lilium & Guyana & Upper Takutu-Upr & $5 \mathrm{Km}$ Se Of Surame 4.1 & -59.05 \\
\hline F36776 & JF455776 & Sturnira lilium & Guyana & Upper Takutu-Upr & $5 \mathrm{Km}$ Se Of Surame 4.1 & -59.05 \\
\hline F36777 & JF455777 & Sturnira lilium & Guyana & Upper Takutu-Upr & $5 \mathrm{Km}$ Se Of Surame 4.1 & -59.05 \\
\hline F36778 & JF455778 & Sturnira lilium & Guyana & Upper Takutu-Upr & $5 \mathrm{Km}$ Se Of Surame 4.1 & -59.05 \\
\hline F36779 & JF455779 & Sturnira lilium & Guyana & Upper Takutu-Upr & $5 \mathrm{Km}$ Se Of Surame 4.1 & -59.05 \\
\hline F36791 & JF455780 & Sturnira lilium & Guyana & Upper Takutu-Upr & $5 \mathrm{Km}$ Se Of Surame 4.1 & -59.05 \\
\hline F38739 & JF455754 & Sturnira lilium & Guyana & Upper Takutu-Upr & Essequibo River, $7 \mid 1.583$ & -58.633 \\
\hline F38765 & JF455755 & Sturnira lilium & Guyana & Upper Takutu-Upr & Gunn'S Strip $\quad 1.65$ & -58.633 \\
\hline F38766 & JF455756 & Sturnira lilium & Guyana & Upper Takutu-Upr & Gunn'S Strip & -58.633 \\
\hline F38767 & JF455757 & Sturnira lilium & Guyana & Upper Takutu-Upr & Gunn'S Strip & -58.633 \\
\hline F38768 & JF455758 & Sturnira lilium & Guyana & Upper Takutu-Upr & Gunn'S Strip & -58.633 \\
\hline F38783 & JF455759 & Sturnira lilium & Guyana & Upper Takutu-Upr & Gunn'S Strip & -58.633 \\
\hline F39795 & JF455761 & Sturnira lilium & Guyana & Cuyuni-Mazaruni & Namai Creek, 5 Km 5.8 & -61.1 \\
\hline F43238 & JF455760 & Sturnira lilium & Guyana & Cuyuni-Mazaruni & Namai Creek, 5 Km 5.8 & -61.1 \\
\hline F43290 & JF455842 & Sturnira lilium & Guyana & Cuyuni-Mazaruni & Namai Creek, 5 Km 5.8 & -61.1 \\
\hline F43291 & JF455843 & Sturnira lilium & Guyana & Cuyuni-Mazaruni & Namai Creek, 5 Km 5.8 & -61.1 \\
\hline F43309 & JF455844 & Sturnira lilium & Guyana & Cuyuni-Mazaruni & Paruima $\quad 5.817$ & -61.067 \\
\hline F43325 & JF455845 & Sturnira lilium & Guyana & Cuyuni-Mazaruni & Paruima $\quad 5.817$ & -61.067 \\
\hline F43601 & JF455846 & Sturnira lilium & Guyana & Potaro-Siparuni & Cow Fly Camp, 40 \& 4.333 & -58.817 \\
\hline F43763 & JF455847 & Sturnira lilium & Guyana & Potaro-Siparuni & Gorge Camp, 40 Kn 4.333 & -58.8 \\
\hline F43803 & JF455753 & Sturnira lilium & Guyana & Potaro-Siparuni & Gorge Camp, 40 Kn 4.333 & -58.8 \\
\hline F46419 & JF455841 & Sturnira lilium & Guyana & Potaro-Siparuni & Mount Ayanganna, 5.333 & -59.917 \\
\hline F50656 & JF455838 & Sturnira lilium & Guyana & Demerara-Mahaic & Ceiba Biological Ce 6.5 & -58.217 \\
\hline F50675 & JF455839 & Sturnira lilium & Guyana & Demerara-Mahaic & Ceiba Biological Ce 6.5 & -58.217 \\
\hline F50814 & JF455840 & Sturnira lilium & Guyana & Demerara-Mahaic & Ceiba Biological Ce 6.5 & -58.217 \\
\hline F50909 & EF080689 & Sturnira lilium & Guyana & Essequibo Islands & Shanklands $\quad 6.483$ & -58.583 \\
\hline F52712 & JF459281 & Sturnira lilium & Guyana & Potaro-Siparuni & Iwokrama Forest, C 4.25 & -58.909 \\
\hline FN31521 & JF455834 & Sturnira lilium & Guyana & Barima-Waini & Kumaka $\quad 7.65$ & -58.95 \\
\hline FN31522 & JF455835 & Sturnira lilium & Guyana & Barima-Waini & Kumaka & -58.95 \\
\hline FN31653 & JF455836 & Sturnira lilium & Guyana & Barima-Waini & Santa Cruz & -59.233 \\
\hline FN31684 & JF455837 & Sturnira lilium & Guyana & Barima-Waini & Waikerebi $\quad 7.517$ & -59.383 \\
\hline FN33331 & JF455819 & Sturnira lilium & Guyana & Upper Takutu-Upr & Kuma River, 5 Mi E, 3.267 & -59.717 \\
\hline FN33332 & JF455820 & Sturnira lilium & Guyana & Upper Takutu-Upr & Kuma River, 5 Mi E: 3.267 & -59.717 \\
\hline FN33333 & JF455821 & Sturnira lilium & Guyana & Upper Takutu-Upr & Kuma River, 5 Mi E, 3.267 & -59.717 \\
\hline FN33334 & JF455822 & Sturnira lilium & Guyana & Upper Takutu-Upr & Kuma River, 5 Mi E, 3.267 & -59.717 \\
\hline FN33363 & JF455823 & Sturnira lilium & Guyana & Upper Takutu-Upr & Kuma River, 5 Mi E, 3.267 & -59.717 \\
\hline FN33364 & JF455824 & Sturnira lilium & Guyana & Upper Takutu-Upr & Kuma River, 5 Mi E, 3.267 & -59.717 \\
\hline FN33365 & JF455825 & Sturnira lilium & Guyana & Upper Takutu-Upr & Kuma River, 5 Mi E, 3.267 & -59.717 \\
\hline FN33396 & JF455826 & Sturnira lilium & Guyana & Upper Takutu-Upr & Karanambo $\quad 3.75$ & -59.3 \\
\hline FN33397 & JF455827 & Sturnira lilium & Guyana & Upper Takutu-Upr & Karanambo & -59.3 \\
\hline FN33398 & JF455828 & Sturnira lilium & Guyana & Upper Takutu-Upr & Karanambo & -59.3 \\
\hline FN33431 & & Sturnira lilium & Guyana & Upper Takutu-Upr & Karanambo & -59.3 \\
\hline FN33447 & JF455829 & Sturnira lilium & Guyana & Upper Takutu-Upr & Karanambo & -59.3 \\
\hline FN33448 & JF455830 & Sturnira lilium & Guyana & Upper Takutu-Upr & Karanambo & -59.3 \\
\hline FN33449 & JF455831 & Sturnira lilium & Guyana & Upper Takutu-Upr & Karanambo & -59.3 \\
\hline FN33450 & JF455832 & Sturnira lilium & Guyana & Upper Takutu-Upr & Karanambo & -59.3 \\
\hline FN33451 & JF455833 & Sturnira lilium & Guyana & Upper Takutu-Upr & Karanambo & -59.3 \\
\hline T-4844 & ABFG452-10 & Sturnira lilium & Martinique & Le Precheur & Case Petit & -61.2064 \\
\hline T-4847 & ABFG455-10 & Sturnira lilium & Martinique & Morne Rouge & Domaine Estripault & \\
\hline T-4928 & HQ918465 ABFG467-10 & Sturnira lilium & Martinique & Morne Rouge & Domaine Estripault & \\
\hline T-4934 & HQ918469 ABFG472-10 & Sturnira lilium & Martinique & Morne Rouge & Domaine Estripault & \\
\hline T-5146 & HQ918485 ABFG497-10 & Sturnira lilium & Martinique & Grand Riviere & Habitation Beausejc & \\
\hline
\end{tabular}


V-2023: N

$\mathrm{V}-2026: \mathrm{N}$

V-2087 HQ918465

V-2093 HQ918469

V-2248 HQ918485

AVB0803: HM208682

AVB0803: HM208683

AVB0803: HM208684

AVB0803: HM208685

FN30074 EF546846

FN30079 JF447329

FN30408 JF447337

FN30421 JF447338

FN30888 EF546837

FN30889 EF546836

FN30904 JF447330

FN30924 JF447331

FN30925 JF447332

FN30926 JF447333

FN32530 JF447336

FN33833 JF447334

FN33836 JF447335

F48087 JF459568

F38203 JF448133

F38204 JF447435

F38205 JF447434

F38218 EF546834

F41153 EF546823

F41154

F41246

F41248

F41249

F41250

F41251

F41252

F41253 EF546822

F41254 EF546821

F41255 EF546820

F41256 EF546819

F41282

F41289

F52792 HQ545490

F52802 HQ545497

F52803 HQ545498

F52953 HQ545626

F53112 HQ919684

F53133 HQ919703

F53135 HQ919705

F53139 HQ919708

F53164 HQ919729

F53170 HQ919734

F53191 HQ919753

F54228 JF447731

F54453 JF447732

F54805 EU097034

F54891 EU097037

F54895 EU097036

F54913 EU097035

F54918 EU097039

F54946 EU097041

F54947 EU097038

F54948 EU097040

F40126 JF449187

F37015 JF455818

F54963 EU097042

F43107 EF546828

F40100 JF448132

F38034 EF546835

FN31834 JF446843

FN30092 EF546845

FN30887 JF447339

BCQCAZ-1122.1

BCQCAZ- JN659889

CACB-05 JN659854

MECN-14 JN659860

MECN-16 JN659869

MECN-20 JN659815

PJV-1018 JN659807

PJV-1019 JN659808

PJV-1020 JN659880
Sturnira lilium

Sturnira lilium

Sturnira lilium

Sturnira lilium

Sturnira lilium

Sturnira lilium

Sturnira lilium

Sturnira lilium

Sturnira lilium

Sturnira lilium

Sturnira lilium

Sturnira lilium

Sturnira lilium

Sturnira lilium

Sturnira lilium

Sturnira lilium

Sturnira lilium

Sturnira lilium

Sturnira lilium

Sturnira lilium

Sturnira lilium

Sturnira lilium

Sturnira lilium

Sturnira lilium

Sturnira lilium

Sturnira lilium

Sturnira lilium

Sturnira lilium

Sturnira lilium

Sturnira lilium

Sturnira lilium

Sturnira lilium

Sturnira lilium

Sturnira lilium

Sturnira lilium

Sturnira lilium

Sturnira lilium

Sturnira lilium

Sturnira lilium

Sturnira lilium

Sturnira lilium

Sturnira lilium

Sturnira lilium

Sturnira lilium

Sturnira lilium

Sturnira lilium

Sturnira lilium

Sturnira lilium

Sturnira lilium

Sturnira lilium

Sturnira lilium

Sturnira lilium

Sturnira lilium

Sturnira lilium

Sturnira lilium

Sturnira lilium

Sturnira lilium

Sturnira lilium

Sturnira lilium

Sturnira lilium

Sturnira lilium

Sturnira lilium

Sturnira lilium 3

Sturnira lilium 3

Sturnira lilium 3

Sturnira lilium 3

Sturnira lilium/luisi

Sturnira lilium/luisi

Sturnira lilium/parvidens

Sturnira lilium/parvidens

Sturnira lilium/parvidens

Sturnira luisi

Sturnira luisi

Sturnira luisi

Sturnira luisi

Sturnira luisi

Sturnira luis

Sturnira luisi

Sturnira luisi

Sturnira luisi

\begin{tabular}{|c|c|c|}
\hline Martinique & Le Precheur & Case Petit $\quad 14.8189$ \\
\hline Martinique & Morne Rouge & Domaine Estripault \\
\hline Martinique & Morne Rouge & Domaine Estripault \\
\hline Martinique & Morne Rouge & Domaine Estripault \\
\hline Martinique & Grand Riviere & Habitation Beausejc \\
\hline Mexico & Campeche & Vicinity of Zoh-Lagu 18.5971 \\
\hline Mexico & Campeche & Vicinity of Zoh-Lagu 18.5971 \\
\hline Mexico & Campeche & Vicinity of Zoh-Lagu 18.5971 \\
\hline Mexico & Campeche & Vicinity of Zoh-Lagu 18.5971 \\
\hline Mexico & Campeche & 3.7 Km Se Of Chekı 18.8 \\
\hline Mexico & Campeche & 3.7 Km Se Of Chekı 18.8 \\
\hline Mexico & Yucatan & Laguna Becanchen 19.883 \\
\hline Mexico & Yucatan & Laguna Becanchen 19.883 \\
\hline Mexico & Quintana Roo & $6 \mathrm{Km} \mathrm{S}$ Of Majahua 18.683 \\
\hline Mexico & Quintana Roo & $6 \mathrm{Km} \mathrm{S}$ Of Majahua 18.683 \\
\hline Mexico & Quintana Roo & $6 \mathrm{Km} \mathrm{S}$ Of Majahua 18.683 \\
\hline Mexico & Quintana Roo & Laguna Noh-Bec, 219.133 \\
\hline Mexico & Quintana Roo & Laguna Noh-Bec, 219.133 \\
\hline Mexico & Quintana Roo & Laguna Noh-Bec, 219.133 \\
\hline Mexico & Quintana Roo & Laguna Noh-Bec, 219.133 \\
\hline Mexico & Quintana Roo & $4 \mathrm{Km} \mathrm{S}$ Of Tulum \\
\hline Mexico & Quintana Roo & $10 \mathrm{Km} \mathrm{N}$ Of Tulum \\
\hline
\end{tabular}

$-89.4222$

$-89.4222$

$-89.4222$

$-89.4222$

$-90.9833$

$-90.983$

$-89.3$

$-87.733$

$-87.733$

$-87.733$

$-88.183$

$-88.183$

$-88.183$

$-88.183$

Darien

Panama

Panama

Panama

Panama

Suriname

Suriname

Suriname

Suriname

Suriname

Suriname

Suriname

Suriname

Suriname

Suriname

Suriname

Suriname

Suriname

Suriname

Suriname

Suriname

Suriname

Suriname

Suriname

Suriname

Suriname

Suriname

Suriname

Suriname

Suriname

Suriname

Suriname

Suriname

Suriname

Suriname

Suriname

Suriname

Suriname

Suriname

Suriname

Ecuador

Guyana

Suriname

Venezuela

Ecuador

Panama

Guatemala

Mexico

Mexico

Ecuador

Ecuador

Ecuador

Ecuador

Ecuador

Ecuador

Ecuador

Ecuador

Ecuador

Estacion Pirre, Parc 8

Estacion Pirre, Parc 8

Estacion Pirre, Parc 8

Estacion Pirre, Parc 8

Brownsberg Nature 4.933

Brownsberg Nature 4.933

Brownsberg Nature 4.933

Brownsberg Nature 4.933

Brownsberg Nature 4.933

Brownsberg Nature 4.933

Brownsberg Nature 4.933

Brownsberg Nature 4.933

Brownsberg Nature 4.933

Brownsberg Nature 4.933

Brownsberg Nature 4.933

Brownsberg Nature 4.933

Brownsberg Nature 4.933

Brownsberg Nature 4.917

Kwamalasamutu $235429-56.789$

Kwamalasamutu 2.35429

Kushere Landing, S $1.944 \quad-56.063$

Iconja Landing, Sipc $1.994 \quad-56.092$

Iconja Landing, Sip $1.994 \quad-56.092$

Iconja Landing, Sip $1.994 \quad-56.092$

Iconja Landing, Sipc $1.994 \quad-56.092$

Iconja Landing, Sip $1.994 \quad-56.092$

Iconja Landing, Sip $1.994 \quad-56.092$

Sipaliwini Village $2.026 \quad-56.124$

Bakhuis, Transect $94.478 \quad-57.042$

Bakhuis, Transect $74.529 \quad-56.904$

Bakhuis, Area 8 Cal $4.542 \quad-56.933$

Blanche Marie Falls $4.811 \quad-56.994$

Blanche Marie Falls $4.811 \quad-56.994$

Blanche Marie Falls $4.811 \quad-56.994$

Blanche Marie Falls $4.811 \quad-56.994$

Blanche Marie Falls 4.811 $\quad-56.994$

Blanche Marie Falls $4.811 \quad-56.994$

Blanche Marie Falls $4.811 \quad-56.994$

Parque Nacional Ya-0.65 $\quad-76.45$

Sipaliwini

Napo

Upper Demerara-I Mabura Hill 5.3

Sipaliwini Blanche Marie Falls $4.811 \quad-56.994$

$3 \mathrm{Km}$ E Of Puerto $\mathrm{C} 7.167$

Esmeraldas $\quad 2 \mathrm{Km} \mathrm{S}$ Of Alto Tam $0.9 \quad-78.55$

Canal Zone

Peten

Tikal

9.1

$-79.7$

Napo 
PJV-1021 JN659881 PJV-1022 JN659866

PJV-1023 JN659809 PJV-1024 JN659810 PJV-1025 JN659811

PJV-268 JN659883

PJV-416 JN659820

PJV-585 JN659856

PJV-592 JN659812

PJV-636 JN659882

PJV-637 JN659813

PJV-641 JN659814

PJV-744 JN659888

PJV-812 JN659858

PJV-820 JN659871

PJV-821 JN659781

PJV-822 JN659782

PJV-823 JN659783

PJV-824 JN659784

PJV-825 JN659785

PJV-828 JN659852

PJV-829 JN659851

PJV-830 JN659850

PJV-831 JN659849

PJV-832 JN659848

PJV-833 JN659847

PJV-834 JN659846

PJV-855 JN659845

PJV-856 JN659874

PJV-858 JN659857

PJV-859 JN659870

PJV-861 JN659786

PJV-863 JN659875

PJV-864 JN659787

PJV-865 JN659788

PJV-895 JN659876

PJV-897 JN659789

PJV-898 JN659877

PJV-899 JN659790

PJV-900 JN659791

PJV-903 JN659878

PJV-904 JN659865

PJV-909 JN659792

PJV-910 JN659793

PJV-911 JN659794

PJV-912 JN659795

PJV-914 JN659796

PJV-915 JN659797

PJV-916 JN659867

PJV-917 JN659872

PJV-918 JN659798

PJV-941 JN659879

PJV-942 JN659799

PJV-943 JN659873

PJV-963 JN659800

PJV-964 JN659801

PJV-965 JN659802

PJV-966 JN659803

PJV-967 JN659804

PJV-968 JN659805

PJV-969 JN659806

PJV-970 JN659868

PJV-971 JN659844

PJV-972 JN659843

PJV-973 JN659842

PJV-974 JN659841

PJV-975 JN659840

PJV-976 JN659839

PJV-977 JN659863

PJV-978 JN659838

PJV-979 JN659864

PJV-980 JN659837

PJV-981 JN659836

PJV-982 JN659835

PJV-983 JN659834

PJV-984 JN659833

PJV-985 JN659832

PJV-986 JN659861

PJV-987 JN659831

PJV-988 JN659830
Sturnira luisi

Sturnira luisi

Sturnira luisi

Sturnira luisi

Sturnira luisi

Sturnira luisi

Sturnira luisi

Sturnira luisi

Sturnira luisi

Sturnira luisi

Sturnira luisi

Sturnira luisi

Sturnira luisi

Sturnira luisi

Sturnira luisi

Sturnira luisi

Sturnira luisi

Sturnira luisi

Sturnira luisi

Sturnira luisi

Sturnira luisi

Sturnira luisi

Sturnira luisi

Sturnira luisi

Sturnira luisi

Sturnira luisi

Sturnira luisi

Sturnira luisi

Sturnira luisi

Sturnira luisi

Sturnira luisi

Sturnira luisi

Sturnira luisi

Sturnira luisi

Sturnira luisi

Sturnira luisi

Sturnira luisi

Sturnira luisi

Sturnira luisi

Sturnira luisi

Sturnira luisi

Sturnira luisi

Sturnira luisi

Sturnira luisi

Sturnira luisi

Sturnira luisi

Sturnira luisi

Sturnira luisi

Sturnira luisi

Sturnira luisi

Sturnira luisi

Sturnira luisi

Sturnira luisi

Sturnira luisi

Sturnira luisi

Sturnira luisi

Sturnira luisi

Sturnira luisi

Sturnira luisi

Sturnira luisi

Sturnira luisi

Sturnira luisi

Sturnira luisi

Sturnira luisi

Sturnira luisi

Sturnira luisi

Sturnira luisi

Sturnira luisi

Sturnira luisi

Sturnira luisi

Sturnira luisi

Sturnira luisi

Sturnira luis

Sturnira luisi

Sturnira luisi

Sturnira luisi

Sturnira luisi

Sturnira luisi

Sturnira luisi

Sturnira luisi

\begin{tabular}{|c|c|c|c|}
\hline Ecuador & Manabi & Parque Nacional $M_{c}-1.53$ & -80.74 \\
\hline Ecuador & Manabi & Parque Nacional $M_{c}-1.53$ & -80.74 \\
\hline Ecuador & Manabi & Parque Nacional $M_{c}-1.53$ & -80.74 \\
\hline Ecuador & Manabi & Parque Nacional $M_{c}-1.53$ & -80.74 \\
\hline Ecuador & Manabi & Parque Nacional $M_{\epsilon}-1.53$ & -80.74 \\
\hline Ecuador & Pichincha & Occidente de Nanes & \\
\hline Ecuador & Orellana & Parque Nacional Ya -0.67 & -76.67 \\
\hline Ecuador & Pichincha & Reserva Otongachi -0.33 & -78.94 \\
\hline Ecuador & Cotopaxi & Santa Rosa $\quad-0.35$ & -78.92 \\
\hline Ecuador & Morona-Santiago & Shell, Fuerte Militar -1.51 & -78.06 \\
\hline Ecuador & Morona-Santiago & Shell, Fuerte Militar -1.51 & -78.06 \\
\hline Ecuador & Morona-Santiago & Shell, Fuerte Militar -1.51 & -78.06 \\
\hline Ecuador & Esmeraldas & Cabo San Franciscc 0.71 & -80.09 \\
\hline Ecuador & Morona-Santiago & Yantzaza $\quad-3.83$ & -78.76 \\
\hline Ecuador & Loja & Cariamanga & -79.55 \\
\hline Ecuador & Loja & Cariamanga & -79.55 \\
\hline Ecuador & Loja & Cariamanga & -79.55 \\
\hline Ecuador & Loja & Cariamanga & -79.55 \\
\hline Ecuador & Loja & Cariamanga & -79.55 \\
\hline Ecuador & Loja & Cariamanga & -79.55 \\
\hline Ecuador & Loja & Cariamanga & -79.55 \\
\hline Ecuador & Loja & Cariamanga & -79.55 \\
\hline Ecuador & Loja & Cariamanga & -79.55 \\
\hline Ecuador & Loja & Cariamanga & -79.55 \\
\hline Ecuador & Loja & Cariamanga & -79.55 \\
\hline Ecuador & Loja & Cariamanga & -79.55 \\
\hline Ecuador & Loja & Cariamanga & -79.55 \\
\hline Ecuador & Loja & Cariamanga & -79.55 \\
\hline Ecuador & Loja & Cariamanga & -79.55 \\
\hline Ecuador & Loja & Cariamanga & -79.55 \\
\hline Ecuador & Loja & Cariamanga & -79.55 \\
\hline Ecuador & Loja & Cariamanga & -79.55 \\
\hline Ecuador & Loja & Cariamanga & -79.55 \\
\hline Ecuador & Loja & Cariamanga & -79.55 \\
\hline Ecuador & Loja & Cariamanga & -79.55 \\
\hline Ecuador & Loja & Cariamanga & -79.55 \\
\hline Ecuador & Loja & Cariamanga & -79.55 \\
\hline Ecuador & Loja & Cariamanga & -79.55 \\
\hline Ecuador & Loja & Cariamanga & -79.55 \\
\hline Ecuador & Loja & Cariamanga & -79.55 \\
\hline Ecuador & Loja & Cariamanga & -79.55 \\
\hline Ecuador & Loja & Cariamanga & -79.55 \\
\hline Ecuador & Loja & Cariamanga & -79.55 \\
\hline Ecuador & Loja & Cariamanga & -79.55 \\
\hline Ecuador & Loja & Cariamanga & -79.55 \\
\hline Ecuador & Loja & Cariamanga & -79.55 \\
\hline Ecuador & Loja & Cariamanga & -79.55 \\
\hline Ecuador & Loja & Cariamanga & -79.55 \\
\hline Ecuador & Loja & Cariamanga & -79.55 \\
\hline Ecuador & Loja & Cariamanga & -79.55 \\
\hline Ecuador & Loja & Cariamanga $\quad-4.32$ & -79.55 \\
\hline Ecuador & Esmeraldas & Quingue, Reserva Iı 0.71 & -80.09 \\
\hline Ecuador & Esmeraldas & Quingue, Reserva lı 0.71 & -80.09 \\
\hline Ecuador & Esmeraldas & Quingue, Reserva Iı 0.71 & -80.09 \\
\hline Ecuador & Manabi & Finca Organica Rio -0.46 & -80.41 \\
\hline Ecuador & Manabi & Finca Organica Rio -0.46 & -80.41 \\
\hline Ecuador & Manabi & Finca Organica Rio -0.46 & -80.41 \\
\hline Ecuador & Manabi & Finca Organica Rio -0.46 & -80.41 \\
\hline Ecuador & Manabi & Finca Organica Rio -0.46 & -80.41 \\
\hline Ecuador & Manabi & Finca Organica Rio -0.46 & -80.41 \\
\hline Ecuador & Manabi & Finca Organica Rio -0.46 & -80.41 \\
\hline Ecuador & Manabi & Finca Organica Rio -0.46 & -80.41 \\
\hline Ecuador & Manabi & Finca Organica Rio -0.46 & -80.41 \\
\hline Ecuador & Manabi & Finca Organica Rio -0.46 & -80.41 \\
\hline Ecuador & Manabi & Finca Organica Rio -0.46 & -80.41 \\
\hline Ecuador & Manabi & Finca Organica Rio -0.46 & -80.41 \\
\hline Ecuador & Manabi & Finca Organica Rio -0.46 & -80.41 \\
\hline Ecuador & Manabi & Finca Organica Rio - 0.46 & -80.41 \\
\hline Ecuador & Manabi & Finca Organica Rio - 0.46 & -80.41 \\
\hline Ecuador & Manabi & Finca Organica Rio -0.46 & -80.41 \\
\hline Ecuador & Manabi & Finca Organica Rio -0.46 & -80.41 \\
\hline Ecuador & Manabi & Finca Organica Rio -0.46 & -80.41 \\
\hline Ecuador & Manabi & Finca Organica Rio -0.46 & -80.41 \\
\hline Ecuador & Manabi & Finca Organica Rio -0.46 & -80.41 \\
\hline Ecuador & Manabi & Finca Organica Rio -0.46 & -80.41 \\
\hline Ecuador & Manabi & Finca Organica Rio -0.46 & -80.41 \\
\hline Ecuador & Manabi & Finca Organica Rio -0.46 & -80.41 \\
\hline Ecuador & Manabi & Finca Organica Rio -0.46 & -80.41 \\
\hline Ecuador & Manabi & Finca Organica Rio -0.46 & -80.41 \\
\hline Ecuador & Manabi & Finca Organica Rio -0.46 & -80.41 \\
\hline
\end{tabular}




\begin{tabular}{|c|c|c|c|c|c|c|c|c|}
\hline PJV-989 & \multicolumn{2}{|c|}{ JN659829 } & Sturnira luisi & Ecuador & Manabi & \multicolumn{2}{|c|}{ Finca Organica Rio -0.46} & -80.41 \\
\hline PJV-990 & \multicolumn{2}{|c|}{ JN659828 } & Sturnira luisi & Ecuador & Manabi & \multicolumn{2}{|c|}{ Finca Organica Rio - 0.46} & -80.41 \\
\hline PJV-991 & \multicolumn{2}{|c|}{ JN659827 } & Sturnira luisi & Ecuador & Manabi & Finca Organic & o - -0.46 & -80.41 \\
\hline PJV-992 & JN65 & & Sturnira luisi & Ecuador & Manabi & Finca Organic & o - -0.46 & -80.41 \\
\hline PJV-993 & JN65 & & Sturnira luisi & Ecuador & Manabi & Finca Organic & o -0.46 & -80.41 \\
\hline PJV-994 & JN65 & & Sturnira luisi & Ecuador & Manabi & Finca Organic & o - -0.46 & -80.41 \\
\hline PJV-995 & JN65 & & Sturnira luisi & Ecuador & Manabi & Finca Organic & o -0.46 & -80.41 \\
\hline PJV-996 & JN65 & & Sturnira luisi & Ecuador & Manabi & Finca Organic & o -0.46 & -80.41 \\
\hline PJV-997 & JN65 & & Sturnira luisi & Ecuador & Manabi & Finca Organic & o -0.46 & -80.41 \\
\hline PJV-998 & JN65 & & Sturnira luisi & Ecuador & Manabi & Finca Organic & o- -0.46 & -80.41 \\
\hline QCAZ-26. & JN65 & & Sturnira luisi & Ecuador & Napo & Archidona & -0.91 & -77.8 \\
\hline QCAZ-26. & JN65 & & Sturnira luisi & Ecuador & Napo & Archidona & -0.91 & -77.8 \\
\hline QCAZ-43 & JN65 & & Sturnira luisi & Ecuador & Orellana & Yasunin, Esta & C -0.67 & -76.67 \\
\hline QCAZ-43 & JN65 & & Sturnira luisi & Ecuador & Orellana & Yasunin, Esta & C -0.67 & -76.67 \\
\hline QCAZ-51: & JN65 & & Sturnira luisi & Ecuador & Napo & Santo Domin & & \\
\hline QCAZ-51: & JN65 & & Sturnira luisi & Ecuador & Napo & Santo Domin & & \\
\hline QCAZ-51 & JN65 & & Sturnira luisi & Ecuador & Napo & Playas Rio Hc & & \\
\hline QCAZ-85 & JN65 & & Sturnira luisi & Ecuador & Pichincha & La Perla, Bos & $\mathrm{PI} 0.13$ & -79.5 \\
\hline RMFN-14 & JN65 & & Sturnira luisi & Ecuador & Orellana & Parque Nacio & Ya-0.67 & -76.67 \\
\hline RMFN-22 & JN65 & & Sturnira luisi & Ecuador & Pastaza & Fuerte Militar & $z-1.51$ & -78.07 \\
\hline SC-6610 & JN65 & & Sturnira luisi & Ecuador & Pichincha & Union del Rio & ct -0.32 & -78.95 \\
\hline & GU72 & & Tadarida brasiliensis & & & & & \\
\hline & GU72 & & Tadarida brasiliensis & & & & & \\
\hline & GU72 & & Tadarida brasiliensis & & & & & \\
\hline & GU72 & & Tadarida brasiliensis & & & & & \\
\hline & GU72 & & Tadarida brasiliensis & & & & & \\
\hline & GU72 & & Tadarida brasiliensis & & & & & \\
\hline & GU72 & & Tadarida brasiliensis & & & & & \\
\hline & GU72 & & Tadarida brasiliensis & & & & & \\
\hline & GU72 & & Tadarida brasiliensis & & & & & \\
\hline & GU72 & & Tadarida brasiliensis & & & & & \\
\hline & GU72 & & Tadarida brasiliensis & & & & & \\
\hline & GU72 & & Tadarida brasiliensis & & & & & \\
\hline & GU72 & & Tadarida brasiliensis & & & & & \\
\hline & GU72 & & Tadarida brasiliensis & & & & & \\
\hline & GU72 & & Tadarida brasiliensis & & & & & \\
\hline & GU72 & & Tadarida brasiliensis & & & & & \\
\hline & GU72 & & Tadarida brasiliensis & & & & & \\
\hline & GU72 & & Tadarida brasiliensis & & & & & \\
\hline & GU72 & & Tadarida brasiliensis & & & & & \\
\hline & GU72 & & Tadarida brasiliensis & & & & & \\
\hline & GU72 & & Tadarida brasiliensis & & & & & \\
\hline & GU72 & & Tadarida brasiliensis & & & & & \\
\hline & GU72 & & Tadarida brasiliensis & & & & & \\
\hline & GU72 & & Tadarida brasiliensis & & & & & \\
\hline & GU72 & & Tadarida brasiliensis & & & & & \\
\hline & GU72 & & Tadarida brasiliensis & & & & & \\
\hline & GU72 & & Tadarida brasiliensis & & & & & \\
\hline & GU72 & & Tadarida brasiliensis & & & & & \\
\hline & GU72 & & Tadarida brasiliensis & & & & & \\
\hline & GU72 & & Tadarida brasiliensis & & & & & \\
\hline & GU72 & & Tadarida brasiliensis & & & & & \\
\hline & GU72 & & Tadarida brasiliensis & & & & & \\
\hline & GU72 & & Tadarida brasiliensis & & & & & \\
\hline & GU72 & & Tadarida brasiliensis & & & & & \\
\hline FN31375 & & ROM 98386 & Tadarida brasiliensis & Guatemala & El Progreso & $10 \mathrm{Km} \mathrm{Nw}$ Of & a) 14.9167 & -90.1 \\
\hline FN31376 & & ROM 98387 & Tadarida brasiliensis & Guatemala & El Progreso & $10 \mathrm{Km} \mathrm{Nw}$ Of & al 14.9167 & -90.1 \\
\hline FN31377 & & ROM 98388 & Tadarida brasiliensis & Guatemala & El Progreso & $10 \mathrm{Km} \mathrm{Nw}$ Of & a 14.9167 & -90.1 \\
\hline FN31378 & & ROM FN313i & Tadarida brasiliensis & Guatemala & El Progreso & $10 \mathrm{Km} \mathrm{Nw}$ Of & a 14.917 & -90.1 \\
\hline FN31379 & & ROM 98390 & Tadarida brasiliensis & Guatemala & El Progreso & $10 \mathrm{Km} \mathrm{Nw}$ Of & a 14.9167 & -90.1 \\
\hline FN31380 & & ROM 98391 & Tadarida brasiliensis & Guatemala & El Progreso & $10 \mathrm{Km} \mathrm{Nw}$ Of & a) 14.9167 & -90.1 \\
\hline T-4927 & HQ91 & 4 ABFG466-10 & Tadarida brasiliensis & Martinique & Morne Rouge & Domaine Estr & & \\
\hline$T-4930$ & HQ91 & 6 ABFG468-10 & Tadarida brasiliensis & Martinique & Morne Rouge & Domaine Estr & & \\
\hline $\mathrm{T}-4931$ & HQ91 & 7 ABFG469-10 & Tadarida brasiliensis & Martinique & Morne Rouge & Domaine Estr & & \\
\hline V-2086 & & $\mathrm{T}-4927$ & Tadarida brasiliensis & Martinique & Morne Rouge & Domaine Estr & & \\
\hline V-2089 & & $\mathrm{T}-4930$ & Tadarida brasiliensis & Martinique & Morne Rouge & Domaine Estr & & \\
\hline V-2090 & & T-4931 & Tadarida brasiliensis & Martinique & Morne Rouge & Domaine Estr & & \\
\hline 1029_MD & LPC & CIBNOR 118 & Tadarida brasiliensis & Mexico & Baja California & I MULEGE & 27.564 & -113.076 \\
\hline 1030_MD & LPC & CIBNOR 118 & Tadarida brasiliensis & Mexico & Baja California & MULEGE & 27.564 & -113.076 \\
\hline 1031_MD & LPC & CIBNOR 863 & Tadarida brasiliensis & Mexico & Baja California & MULEGE & 27.564 & -113.076 \\
\hline 1032_MD & LPC & CIBNOR 863 & Tadarida brasiliensis & Mexico & Baja California & $\mu$ MULEGE & 27.564 & -113.076 \\
\hline 1033_MD & LPC & CIBNOR 863 & Tadarida brasiliensis & Mexico & Baja California & u MULEGE & 27.564 & -113.076 \\
\hline 1034_MD & LPC & CIBNOR 863 & Tadarida brasiliensis & Mexico & Baja California & M MULEGE & 27.564 & -113.076 \\
\hline 1047_MD & LPC & CIBNOR 863 & Tadarida brasiliensis & Mexico & Baja California & u MULEGE & 27.564 & -113.076 \\
\hline 1162 PCC & & CIBNOR 117 & Tadarida brasiliensis & Mexico & Baja California & ـ COMONDU & 26.183 & -112.088 \\
\hline 1422_MD & LPC & CIBNOR 109 & Tadarida brasiliensis & Mexico & Baja California & 」 LA PAZ & 24.1431 & -110.429 \\
\hline 4532_STA & & CIBNOR 411 & Tadarida brasiliensis & Mexico & Coahuila & OCAMPO & 27.321 & -102.418 \\
\hline 4536_STA & & CIBNOR 411 & Tadarida brasiliensis & Mexico & Coahuila & OCAMPO & 27.321 & -102.418 \\
\hline 4901_STA & & CIBNOR 539 & Tadarida brasiliensis & Mexico & Puebla & Jardin Botani & a) 18.3272 & -97.4497 \\
\hline 4902_STA & & CIBNOR 539 & Tadarida brasiliensis & Mexico & Puebla & Jardin Botani & a) 18.3272 & -97.4497 \\
\hline
\end{tabular}


samples analyzed as part of the DNA barcoding of Caribbean bats. 